\title{
A variational approach to the mean field planning problem
}

\author{
Carlo Orrieri * $\quad$ Alessio Porretta ${ }^{\dagger} \quad$ Giuseppe Savaré ${ }^{\ddagger}$
}

July 27, 2018

\begin{abstract}
We investigate a first-order mean field planning problem of the form

$$
\left\{\begin{aligned}
-\partial_{t} u+H(x, D u) & =f(x, m) & & \text { in }(0, T) \times \mathbb{R}^{d}, \\
\partial_{t} m-\nabla \cdot\left(m H_{\boldsymbol{p}}(x, D u)\right) & =0 & & \text { in }(0, T) \times \mathbb{R}^{d}, \\
m(0, \cdot)=m_{0}, m(T, \cdot) & =m_{T} & & \text { in } \mathbb{R}^{d},
\end{aligned}\right.
$$
\end{abstract}

associated to a convex Hamiltonian $H$ with quadratic growth and a monotone interaction term $f$ with polynomial growth.

We exploit the variational structure of the system, which encodes the first order optimality condition of a convex dynamic optimal entropy-transport problem with respect to the unknown density $m$ and of its dual, involving the maximization of an integral functional among all the subsolutions $u$ of an Hamilton-Jacobi equation.

Combining ideas from optimal transport, convex analysis and renormalized solutions to the continuity equation, we will prove existence and (at least partial) uniqueness of a weak solution $(m, u)$. A crucial step of our approach relies on a careful analysis of distributional subsolutions to Hamilton-Jacobi equations of the form $-\partial_{t} u+H(x, D u) \leq \alpha$, under minimal summability conditions on $\alpha$, and to a measure-theoretic description of the optimality via a suitable contact-defect measure. Finally, using the superposition principle, we are able to describe the solution to the system by means of a measure on the path space encoding the local behavior of the players.

\section{Contents}

1 Introduction

2 Notation and assumptions 6

2.1 Notation . . . . . . . . . . . . . . . . . . . . . . . . 6

2.2 Structural assumptions . . . . . . . . . . . . . . . . . . . . . . . . 7

3 Optimal transport distance, displacement interpolation, and continuity equation 9

3.1 Recaps on Optimal Transport and dynamic formulation . . . . . . . . . . . . . . 9

3.2 $L^{p}$ probability densities with finite action and the Kantorovich-Lebesgue interpolation cost . 10

4 Convergence in measure, increasing functions, weighted $L^{p}$ spaces and anisotropic convolution

4.1 The space $L^{0}(\Omega ; \mathfrak{m})$ and the convergence in measure . . . . . . . . . . . . . . . . 13

4.2 Properties of increasing functions . . . . . . . . . . . . . . . . . . . . . . . . . 14

4.3 Convolution by anisotropic kernels . . . . . . . . . . . . . . . . . . . . . . . . . 19

4.4 The space $L^{q}+L_{1 / \kappa}^{\infty}(\Omega) \ldots \ldots \ldots \ldots \ldots \ldots \ldots \ldots \ldots \ldots \ldots$

*Dipartimento di Matematica "G. Castelnuovo", Sapienza Università di Roma. Piazzale Aldo Moro 5, 00185 Roma, Italy. The author acknowledges the financial support provided by PRIN 20155PAWZB "Large Scale Random Structures". Email: orrieri@mat.uniroma1.it

${ }^{\dagger}$ Dipartimento di Matematica, Università di Roma Tor Vergata. Via della Ricerca Scientifica 1, 00133 Roma, Italy. Partially supported by University of Tor Vergata ("Consolidate The foundations 2015") project Irreversibility in Dynamic Optimization. Email: porretta@mat.uniroma2.it

¥Dipartimento di Matematica "F. Casorati", Università di Pavia. Via Ferrata 5, 27100 Pavia, Italy. Partially supported by Cariplo foundation and Regione Lombardia via project Variational evolution problems and optimal transport, by MIUR PRIN 2015 project Calculus of Variations, and by IMATI-CNR. Email: giuseppe.savare@unipv.it 
5.1 Weak subsolutions, precise representative and truncations . . . . . . . . . . . . . . 21

5.2 A priori estimates and stability for weak subsolutions . . . . . . . . . . . . . . . 23

5.3 A general duality-transport result . . . . . . . . . . . . . . . . . . . . . . 28

5.4 Contact-defect measures associated to weak subsolutions . . . . . . . . . . . . . . . . 30

6 A Variational approach to MFPP

6.1 The variational structure of the primal problem . . . . . . . . . . . . . . . . 33

6.2 The variational structure of the dual problem and the minimax principle . . . . . . . . . . 37

6.3 Existence of a solution to the dual problem . . . . . . . . . . . . . . . 39

6.4 Optimality conditions and the weak formulation of the Mean Field planning system . . . . . 39

\section{Optimal plans: a Lagrangian viewpoint}

7.1 Continuity equation and measures on the space of continuous curves . . . . . . . . . . . . . 42

7.2 Lifting functions to $\Gamma$ and regularization of dynamic plans . . . . . . . . . . . . . . . . . . . . . . . . . . . . . . .

7.3 Modified Lagrangian cost and optimal dynamic plans . . . . . . . . . . . . . . . . . . 46

\section{A Appendix}

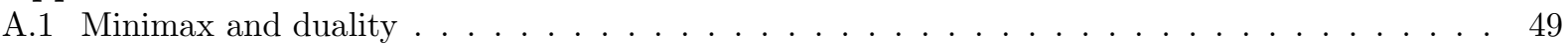

A.2 Convergence in measure . . . . . . . . . . . . . . . . . . . . . . . . 50

A.3 Positive bilinear functionals . . . . . . . . . . . . . . . . . . . . . . . 50

\section{Introduction}

Mean field games, as well as mean field control problems, describe strategic interactions among large numbers of similar rational agents. Typically, the generic agent aims at optimizing some functional depending both on its own (controlled) dynamical state and on the average collective behavior, usually represented by the distribution law of the states. When the individual optimization and the collective evolution are consistent, the system is described by two PDEs satisfied, respectively, by the value function $u$ of the single agent and by the distribution law $m$ of the population. The simplest model is the following coupling of HamiltonJacobi-Bellman and Kolmogorov Fokker-Planck equations:

$$
\left\{\begin{aligned}
-\partial_{t} u-\varepsilon \Delta u+H(x, D u) & =f(x, m), \\
\partial_{t} m-\varepsilon \Delta m-\nabla \cdot\left(m H_{p}(x, D u)\right) & =0
\end{aligned}\right.
$$

where $\varepsilon=0$ and $\varepsilon>0$ distinguish whether the agent's dynamic is purely deterministic or, respectively, it contains some Brownian noise.

Since the introduction of mean field game theory by J.-M. Lasry and P.-L. Lions, who derived (1.1) in connection with the limit of Nash equilibria of $N$-players games as $N \rightarrow \infty$ (see [18, 19]), this kind of systems has been extensively studied, mainly under the stabilization condition of $f(x, m)$ being nondecreasing with respect to $m$. The system is usually closed with an initial condition for the density $m$ and a terminal condition (a final pay-off) for the value function $u$.

Otherwise, the planning problem consists in prescribing both initial and terminal conditions for the density $m$. In this case, the goal is to solve the following:

$$
\left\{\begin{aligned}
-\partial_{t} u-\varepsilon \Delta u+H(x, D u) & =f(x, m), \\
\partial_{t} m-\varepsilon \Delta m-\nabla \cdot\left(m H_{p}(x, D u)\right) & =0 \\
m(0)=m_{0}, m(T) & =m_{1}
\end{aligned}\right.
$$

in some finite horizon $T>0$.

In the framework of mean field game theory, the planning problem was suggested and developed by P.-L. Lions in his courses at Collège de France. In particular, when the problem is set on the flat torus and smooth initial-terminal densities $m_{0}, m_{1}$ are prescribed, P.-L. Lions proved the existence of smooth solutions both for the second order case $(\varepsilon>0$ in (1.2) $)$ with quadratic Hamiltonian $H(x, p)$ and for the first order case $(\varepsilon=0$ ) when $f=f(m)$ is an increasing function (see [23]). Later, existence and uniqueness of weak solutions were proved for the second order case for more general Hamiltonians (see [27, 28]). Here the strategy used was to build solutions of the planning problem by penalizing the final pay-off $u(T)$ in a way to force the required density condition $m(T)$ to hold at the final time. This approach, which was also exploited for numerical schemes in [1], reminds that exact controllability in finite time can be obtained as singular limit of optimal control problems through penalization of the final pay-off, and therefore of the adjoint state at the final time. Indeed, J.-M. Lasry and P.-L. Lions already addressed, in their initial papers on mean field 
games (see [19]), that system (1.2) can be recast as the optimality system satisfied by state and adjoint state of an optimal control problem. Precisely, if $F(x, m)=\int_{0}^{m} f(x, s) d s$ and $L(x, \boldsymbol{q})$ is the Fenchel conjugate of the Hamiltonian $H(x,-\boldsymbol{p})$, system (1.1) formally appears as the first order condition of the following minimization problem:

$$
\min \int_{0}^{T} \int_{\mathbb{R}^{d}}[L(x, \boldsymbol{v}) m+F(x, m)] \mathrm{d} x \mathrm{~d} t: \quad \boldsymbol{v} \in L^{2}(m d x d t), \quad\left\{\begin{array}{r}
\partial_{t} m-\varepsilon \Delta m+\nabla \cdot(m \boldsymbol{v})=0 \\
m(0, \cdot)=m_{0}, m(T, \cdot)=m_{1}
\end{array}\right.
$$

It is well-known that, when $\varepsilon=0, F=0$ and $H(x, \boldsymbol{p})=\frac{1}{2}|\boldsymbol{p}|^{2}$, (1.3) is the so-called fluid mechanics formulation of the Monge-Kantorovich mass transfer problem introduced by Benamou and Brenier (see [4]) and leading to the dynamic characterization of the $L^{2}$-Kantorovich-Rubinstein-Wasserstein distance $W_{2}$ between probability measures in $\mathbb{R}^{d}$ with finite quadratic moment [3, 31]. This approach has then been extended to general Lagrangian formulations (see e.g. [6] and 31, Chap. 7]), also exploiting the metricRiemannian viewpoint intrinsic to the dynamic approach [26, 25, 3 ].

This way, the planning problem (1.2) appears as a natural generalization of optimal transport problems, when the extra penalization term of entropic type (induced by the convex function $F$ in (1.3)) prevents concentration of the transported density $m$.

The study of various kind of entropic relaxation of genuine optimal transport problems recently attracted a lot of attention, due to the regularizing and convexification effect added by the entropic terms (see e.g. [21, 15, 22]). In the present case, since $F$ is nonlinear w.r.t. $m$ (we will consider a typical power behavior of exponent $p \in(1,+\infty)$ ), the minimization of the dynamic cost functional cannot be reduced to a simpler and more explicit Kantorovich formulation involving transport plans, but it has to take into account a complex interpolation dynamic interaction between the transport and the local density terms.

In this article, our goal is to use some ideas of optimal transport theory, convex duality, and dynamic superposition principles in order to study a suitable weak formulation of the deterministic mean field planning problem, that in strong form and assuming $m>0$ everywhere can be formally written as

$$
\left\{\begin{aligned}
-\partial_{t} u+H(x, D u) & =f(x, m)) & & \text { in } Q, \\
\partial_{t} m-\nabla \cdot\left(m H_{\boldsymbol{p}}(x, D u)\right) & =0 & & \text { in } Q \\
m(t, \cdot)=m_{0}, m(1, \cdot) & =m_{1} & & \text { in } \mathbb{R}^{d},
\end{aligned}\right.
$$

where the final time has been normalized to $T=1$ and $Q$ is the space-time cylinder $(0,1) \times \mathbb{R}^{d}$.

Compared to previous results for mean field games systems, we set the problem in the whole space, which seems more natural in the viewpoint of optimal transport of probability measures. Otherwise, as in previous results we rely on two crucial structure conditions, namely that $H(x, \boldsymbol{p})$ is convex with respect to $\boldsymbol{p}$ and $f(x, m)$ is increasing with respect to $m$. The main result that we prove is the existence of weak solutions (and partial uniqueness for $m$ and $D u$ ) to the planning problem (s-MFPP) under fairly general growth conditions on $H$ and $f$, that will be discussed later in detail. The main effect of this generality is that no standard framework can be applied, in particular, to the Hamilton-Jacobi equation.

Let us stress that, in optimal transport theory, the duality between the continuity equation and the Hamilton-Jacobi equation has been mostly exploited formally or under conditions of regularity which allow for the use of explicit representation formula of Hopf-Lax type (typical of the viscosity solutions' theory). Another source of difficulty lies on the possible vanishing of the density $m$ in large sets, so that the first equation of (S-MFPP should be written in a relaxed form.

Unfortunately, the coupling appearing in the system s-MFPP often leads outside the standard framework of continuous solutions to Hamilton-Jacobi equations. By contrast, convex duality methods have been successfully used in mean field game theory under very general growth conditions. In particular, existence and uniqueness results were proved for the deterministic, or degenerate diffusion case, using relaxed solutions of the Hamilton-Jacobi equations and weak formulations of the system, see [9, 11, 12]. Those relaxed formulations appear very naturally from the convex duality of the variational problems, and we will follow a similar strategy here when dealing with the planning problem.

\section{A heuristic derivation of MFPP from the minimax principle}

Before describing the main contributions of our paper, let us first briefly explain the heuristic derivation of the relaxed formulation of (s-MFPP starting from the minimization of the functional

$$
\begin{gathered}
\mathcal{B}(m, \boldsymbol{v}):=\iint_{Q}[L(x, \boldsymbol{v}) m+F(x, m)] \mathrm{d} x \mathrm{~d} t \quad \text { among all the solutions of } \\
\partial_{t} m+\nabla \cdot(m \boldsymbol{v})=0 \quad \text { in } \mathscr{D}^{\prime}\left((0,1) \times \mathbb{R}^{d}\right), \quad m(0, \cdot)=m_{0}, m(1, \cdot)=m_{1}, \quad m \geq 0 .
\end{gathered}
$$


Arguing as in the formal discussion in 25], we write the above constraint minimization as an inf-sup formulation of a saddle problem, where a new Lagrange multiplier $u \in C_{c}^{1}\left(\mathbb{R} \times \mathbb{R}^{d}\right)$ is used to impose the continuity equation and the boundary conditions:

$$
\inf _{m \geq 0, \boldsymbol{v}} \sup _{u} \iint_{Q}[L(x, \boldsymbol{v}) m+F(x, m)] \mathrm{d} x \mathrm{~d} t+\int_{\mathbb{R}^{d}} u_{0} m_{0} \mathrm{~d} x-\int_{\mathbb{R}^{d}} u_{1} m_{1} \mathrm{~d} x+\iint_{Q}\left[\partial_{t} u+D u \cdot \boldsymbol{v}\right] m \mathrm{~d} x \mathrm{~d} t
$$

A standard trick to substitute $\boldsymbol{v}$ with the new variable $\boldsymbol{w}=m \boldsymbol{v}$, so that the saddle function is convex in $(m, \boldsymbol{w})$ and concave (in fact, linear) in $u$, suggests the possibility to interchange the order of inf and sup, obtaining the dual problem

$$
\begin{aligned}
\sup _{u} & \int_{\mathbb{R}^{d}} u_{0} m_{0} \mathrm{~d} x-\int_{\mathbb{R}^{d}} u_{1} m_{1} \mathrm{~d} x+\inf _{m \geq 0, \boldsymbol{v}} \iint_{Q}\left[L(x, \boldsymbol{v}) m+F(x, m)+m \partial_{t} u+m D u \cdot \boldsymbol{v}\right] \mathrm{d} x \mathrm{~d} t \\
& =\sup _{u} \int_{\mathbb{R}^{d}} u_{0} m_{0} \mathrm{~d} x-\int_{\mathbb{R}^{d}} u_{1} m_{1} \mathrm{~d} x+\inf _{m \geq 0} \iint_{Q}\left[\left(-H(x, D u)+\partial_{t} u\right) m+F(x, m)\right] \mathrm{d} x \mathrm{~d} t \\
& =\sup _{u} \int_{\mathbb{R}^{d}} u_{0} m_{0} \mathrm{~d} x-\int_{\mathbb{R}^{d}} u_{1} m_{1} \mathrm{~d} x-\iint_{Q} F^{*}\left(-\partial_{t} u+H(x, D u)\right) \mathrm{d} x \mathrm{~d} t .
\end{aligned}
$$

where by Fenchel duality

$$
\inf _{\boldsymbol{v}} L(x, \boldsymbol{v})+\boldsymbol{p} \cdot \boldsymbol{v}=-H(x, \boldsymbol{p}), \quad \inf _{m \geq 0} F(x, m)+\ell m=-F^{*}(x, \ell) .
$$

The dual problem thus consists in the maximization of

$$
\begin{gathered}
\mathcal{A}(u, \ell):=\int_{\mathbb{R}^{d}} u_{0} m_{0} \mathrm{~d} x-\int_{\mathbb{R}^{d}} u_{1} m_{1} \mathrm{~d} x-\iint_{Q} F^{*}(\ell(t, x)) \mathrm{d} x \mathrm{~d} t, \\
\text { under the constraint }-\partial_{t} u+H(x, D u)=\ell \quad \text { in } Q .
\end{gathered}
$$

Minimax principle yields $\mathcal{B}(m, \boldsymbol{v}) \geq \mathcal{A}(u, \ell)$ whenever $m, \boldsymbol{v}$ solve the continuity equation (1.4) and $u, \ell$ are linked by the Hamilton-Jacobi equation (1.5). The variational formulation of MFPP should then arise as the optimality condition at minimizers $(m, \boldsymbol{v})$ of the primal problem (1.4) and maximizers $(u, \ell)$ of the dual problem (1.5), if there is no duality gap. Such conditions can be easily obtained by rearranging the (nonnegative) difference $\mathcal{B}(m, \boldsymbol{v})-\mathcal{A}(u, \ell)$ and assuming enough regularity in order to justify integration by parts; we obtain the sum of two nonnegative terms:

$$
\mathcal{B}(m, \boldsymbol{v})-\mathcal{A}(u, \ell)=\iint_{Q}(L(x, \boldsymbol{v})+D u \cdot \boldsymbol{v}+H(x, D u)) m \mathrm{~d} x \mathrm{~d} t+\iint_{Q}\left(F(x, m)-\ell m+F^{*}(x, \ell)\right) \mathrm{d} x \mathrm{~d} t
$$

so that, assuming differentiability of $H$ and continuity of $f$, optimal pairs $(m, \boldsymbol{v})$ and $(u, \ell)$ with $\mathcal{B}(m, \boldsymbol{v})=$ $\mathcal{A}(u, \ell)$ are characterized by

$$
\begin{aligned}
&(L(x, \boldsymbol{v})+D u \cdot \boldsymbol{v}+H(x, D u)) m=0 \mathscr{L}^{d+1} \text { a.e. in } Q, \text { i.e. } \boldsymbol{v}=-H_{\boldsymbol{p}}(x, D u) \quad m \text { a.e. in } Q \\
& F(x, m)-\ell m+F^{*}(x, \ell)=0 \mathscr{L}^{d+1} \text { a.e. in } Q, \quad \text { i.e. } \begin{cases}\ell=f(x, m) & \text { if } m>0 \\
\ell \leq f(x, 0) & \text { if } m=0 .\end{cases}
\end{aligned}
$$

Combining (1.6) and (1.7) with the continuity equation of (1.4) and the Hamilton-Jacobi equation of (1.5) we end up with the relaxed formulation of MFPP:

$$
\left\{\begin{aligned}
-\partial_{t} u+H(x, D u) & \leq f(x, m) & & \text { in } Q \\
-\partial_{t} u+H(x, D u) & =f(x, m) & & \text { if } m(x, t)>0 \\
\partial_{t} m-\nabla \cdot\left(m H_{\boldsymbol{p}}(x, D u)\right) & =0 & & \text { in } Q \\
m(t, \cdot)=m_{0}, m(1, \cdot) & =m_{1} & & \text { in } \mathbb{R}^{d} .
\end{aligned}\right.
$$

One can notice that $u$ is just required to be a global subsolution to the Hamilton-Jacobi equation with right-hand side $f(x, m)$; the equality will be attained only in the set where $m$ is strictly positive.

\section{The variational setting and the weak formulation of MFPP}

Since r-MFPP represents the optimality system of a saddle point problem, there is a natural strategy to prove the existence of a solution and to obtain a well posed weak formulation: 
S1. By the Direct method of the Calculus of Variations prove the existence of a minimizer for the primal problem (1.4): it can be formulated as the minimum of two convex and lower semicontinuous functions along curves of probability measures solving the continuity equation. This part will be developed in Section 6.1, a preliminary discussion, related to the particular case when $H(x, \boldsymbol{p})=\frac{1}{2}|p|^{2}$ and $F(x, m)=$ $\frac{1}{2} m^{p}$ (leading to the Kantorovich-Lebesgue $K L_{2, p}$ cost) is developed in Section 3.2

S2. By (a suitably refined version of) the Von Neumann minimax principle (see A.1 in the Appendix) prove that there is no duality gap and $\min \mathcal{B}(m, \boldsymbol{v})=\sup \mathcal{A}(u, \ell)$ in a suitable class of smooth functions (Section 6.2).

S3. As it is typical in Optimal Transport problems, existence of maximizers of the dual problem is a much subtler issue, due to the lack of compactness of the dual formulation in spaces of smooth functions. Here the first equation (in fact an inequality) of the relaxed formulation suggests to first study subsolutions to the Hamilton-Jacobi equation of the form

$$
-\partial_{t} u+H(x, D u) \leq \alpha
$$

just requiring minimal summability on $\alpha$ (derived by an a priori estimate in $L^{q}(Q)$ due to the growth of $F^{*}$ ) and minimal regularity of $u$, in order to give a distributional sense to (1.8).

This preliminary study is the main topic of Section 5. we will recover a suitable notion of traces of $u$ at $t=0$ and $t=1$ in 55.1 we will prove that subsolutions exhibit a nice regularization effect, sufficient to gain upper semicontinuity of $\mathcal{A}$ and enough compactness (Section 5.2), to prove existence of a maximizer of the relaxed formulation (Section 6.3):

$$
\max \left\{\mathcal{A}(u, \alpha):-\partial_{t} u+H(x, D u) \leq \alpha \text { in } \mathscr{D}^{\prime}(Q)\right\} .
$$

It is worth noticing that (1.9) involves a convex constraint on pairs $(u, \alpha)$, which is clearly more stable than the condition $-\partial_{t} u+H(x, D u)=\ell$.

S4. A crucial point concerns the duality between the primal and the relaxed dual problem, in particular the fact that the optimal value of (1.9) still coincides with the minimum of $\mathcal{B}$. This fact will be addressed in sections 5.3 and 6.4 .

S5. Having at our disposal minimizers of $\mathcal{B}$ and maximizers of $\mathcal{A}$, it is not difficult to check that the optimality condition yields $\boldsymbol{v}=-H_{\boldsymbol{p}}(x, D u)$ a.e. on the set where $m>0$ and $\alpha=f(x, m)$. The last technical question concerns the "contact" condition $-\partial_{t} u+H(x, D u)=f(x, m)$ when $m>0$, since we have just a distributional subsolution to the Hamilton-Jacobi equation and $-\partial_{t} u$ may have singular parts (see also [10] for similar questions).

In order to overcome this difficulty, we derive a distributional condition which can be formally obtained by combining the contact condition with the continuity equation satisfied by $m$ : assuming regular solutions, and multiplying the continuity equation by $u$, one can easily obtain

$$
\begin{aligned}
\partial_{t}(u m)+\nabla \cdot(u m \boldsymbol{v}) & =u\left(\partial_{t} m+\nabla \cdot(m \boldsymbol{v})\right)+\left(\partial_{t} u+D u \cdot \boldsymbol{v}\right) m \\
& =\left(\partial_{t} u-H(x, D u)+\alpha\right) m+(-\alpha+H(x, D u)+D u \cdot \boldsymbol{v}) m \\
& =(-\alpha+H(x, D u)+D u \cdot \boldsymbol{v}) m
\end{aligned}
$$

so that we can in principle substitute the contact condition with a suitable (distributional and renormalized) version of

$$
\partial_{t}(u m)+\nabla \cdot(u m \boldsymbol{v})+(\alpha-H(x, D u)-D u \cdot \boldsymbol{v}) m=0 \quad \text { in } Q .
$$

In Section 5.4 we will associate a nonnegative Radon "contact-defect" measure $\vartheta$ to every pair of competitors $(m, \boldsymbol{v})$ for the primal problem and $(u, \alpha)$ for the dual problem and we will show that vanishing of $\vartheta$ is the right distributional way to impose the missing contact condition.

S6. With all the above tools at disposal, Section 6.4 collects all the main result concerning the formulation, the existence and the characterizations of solutions to (r-MFPP).

S7. A further analysis, carried out in the last Section 7. concerns the Lagrangian viewpoint to (r-MFPP). Using the superposition principle, we are able to describe the solutions to r-MFPP by means of a measure $\boldsymbol{\eta}$ on the path space $\mathrm{AC}^{2}\left([0,1] ; \mathbb{R}^{d}\right)$ (the characteristics associated to the velocity vector field $\boldsymbol{v}) . \boldsymbol{\eta}$ encodes the local behaviour of particles (or agents) which try to minimize a modified Lagrangian cost obtained by the sum of $L$ with a potential induced by the mass distribution $\alpha=f(x, m)$.

In this way, we can also recover a "static" description in terms of Optimal Transport, but where the transportation cost is affected by the density $m$ of the moving particles. 
Some preliminary material, concerning Optimal transport, displacement interpolation, continuity equation weighted spaces, convergence in measure and precise representatives of increasing functions is collected in Sections 3 and 4 .

\section{Notation and assumptions}

In this section we collect some notions and results concerning convex functionals on measures, continuity equations and their connections with the theory of optimal transportation. Moreover, we resume some useful properties of locally increasing functions which we will need in the sequel.

\section{$2.1 \quad$ Notation}

\section{List of main notation}

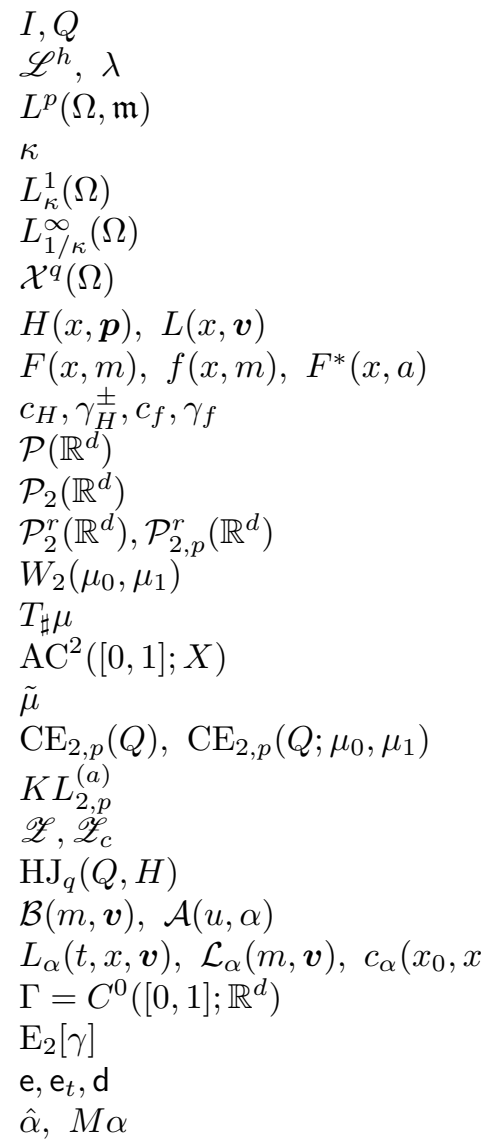

the interval $(0,1)$ and the space-time cylinder $(0,1) \times \mathbb{R}^{d}$

the $h$-dimensional Lebesgue measure and its restriction to $Q$

Lebesgue space w.r.t. the $\sigma$-finite Borel measure $\mathfrak{m}$

the weight $1+|x|^{2}$ on $\mathbb{R}^{d}$ or on $Q$

weighted Lebesgue space of functions $f$ satisfying $f \cdot \kappa \in L^{1}(\Omega)$

the dual space of $L_{\kappa}^{1}(\Omega): f \cdot 1 / \kappa \in L^{\infty}(\Omega)$

the space $L^{q}+L_{1 / \kappa}^{\infty}(\Omega)$ see Definition 4.7

the Hamiltonian and the dual Lagrangian, see 2.1 and (2.19)

the cost density function, its derivative, see 2.1 and (2.14)

structural constants and functions related to $H, f$, see 2.1

the set of Borel probability measure on $\mathbb{R}^{d}$

Borel probability measures with finite quadratic moment

Absolutely continuous measures in $\mathcal{P}_{2}\left(\mathbb{R}^{d}\right)$ (with density in $L^{p}\left(\mathbb{R}^{d}\right)$ )

the Kantorovich-Rubinstein-Wasserstein distance, see (3.1)

push forward of a measure $\mu$ through the map $T$, see Section 3

absolutely continuous curves with values in the metric space $X$

measure in $Q$ whose disintegration is $\mu \in \mathrm{AC}^{2}\left([0,1] ; \mathcal{P}_{2}\left(\mathbb{R}^{d}\right)\right)$, see (3.9)

pairs $(m, \boldsymbol{v})$ solving the continuity equation, Def. 3.3

Kantorovich-Lebesgue costs, Definition 3.4

collections of pairs $(\zeta, Z)$ satisfying (4.11)

subsolutions $(u, \alpha)$ of the Hamilton-Jacobi equations, Def. 5.7

primal and dual functionals, 6.1 and 6.30)

modified Lagrangians and induced transport cost (6.59), (6.60), (7.46)

space of continuous curves with the uniform topology

energy of a curve $\gamma \in \mathrm{AC}_{2}\left([0,1] ; \mathbb{R}^{d}\right),(7.2)$

evaluation maps on $[0,1] \times \Gamma,(7.3),(7.4)$

precise representative and maximal function of $\alpha \in L^{q}(Q),(17.24),(7.25)$

Throughout the paper $I$ stands for the open interval $(0,1)$ and $Q:=(0,1) \times \mathbb{R}^{d}$.

If $\Omega$ is a Polish topological space (i.e. its topology is induced by a complete and separable distance) we will denote by $\mathscr{B}$ its Borel $\sigma$-algebra and by $\mathcal{P}(\Omega)$ the set of Borel probability measures on $\Omega$, endowed with the topology of weak convergence, in duality with the set of all continuous and bounded functions, denoted by $C_{b}(\Omega)$. When $\Omega=\mathbb{R}^{d}$ we will also deal with the space $\mathcal{P}_{2}\left(\mathbb{R}^{d}\right)$ of measures with finite quadratic moment, i.e.

$$
\mu \in \mathcal{P}_{2}\left(\mathbb{R}^{d}\right) \quad \Leftrightarrow \quad \int_{\mathbb{R}^{d}}|x|^{2} \mathrm{~d} \mu(x)<+\infty .
$$

If $\mathfrak{m}$ is a $\sigma$-finite measure on $\Omega, L^{r}\left(\Omega, \mathfrak{m} ; \mathbb{R}^{d}\right)\left(L^{r}(\Omega, \mathfrak{m})\right.$ if $\left.d=1\right) r \in[1, \infty]$, will be the usual Lebesgue space of (classes of) $\mathfrak{m}$-measurable and $\mathbb{R}^{d}$-valued maps $r$-integrable w.r.t $\mathfrak{m}$. We will also use the notation $L^{0}(\Omega)$ for the set of (classes of) measurable real functions. Our typical examples of $\Omega$ consist in Borel subsets of some Euclidean space $\mathbb{R}^{h}$ or in the space of continuous curves $\Gamma:=\mathrm{C}^{0}\left([0,1] ; \mathbb{R}^{d}\right)$.

When $\Omega$ is a Borel subset of $\mathbb{R}^{h}$ (typically $I, \mathbb{R}^{d}$ or $Q$ ), we will denote by $\mathscr{L}^{h}$ the (restriction of the) $h$-dimensional Lebesgue measure and when $\mathfrak{m}=\mathscr{L}^{h}$ we will simply write $L^{r}\left(\Omega ; \mathbb{R}^{d}\right)\left(L^{r}(\Omega)\right.$ when $\left.d=1\right)$. In the particular case of $Q$ we will also use the symbol $\lambda:=\left.\mathscr{L}^{d+1}\right|_{Q}$. If $\Omega$ is open, the set of $r$-integrable maps on compact subsets of $\Omega$ will be denoted by $L_{\text {loc }}^{r}(\Omega)$. 
To every probability density

$$
m \in L^{1}(\Omega) \quad \text { with } \quad m \geq 0 \text { a.e. in } \Omega \quad \text { and } \quad \int_{\Omega} m(x) \mathrm{d} x=1
$$

we can associate a Borel probability measure $\mu \in \mathcal{P}(\Omega)$ by $\mu=m \mathscr{L}^{h}$. Conversely, if $\mu \in \mathcal{P}(\Omega)$ is absolutely continuous w.r.t. $\mathscr{L}^{h}$ then its Lebesgue density $m=\mathrm{d} \mu / \mathrm{d} \mathscr{L}^{h}$ satisfies (2.2). In this way, we will often switch between properties stated on densities $m$ in the convex set of $L^{1}(\Omega)$ characterized by (2.2) and analogous statements for measures $\mu=m \mathscr{L}^{h}$ in the space $\mathcal{P}^{r}(\Omega)$ of Borel probability measures absolutely continuous w.r.t. $\mathscr{L}^{h}$.

If $\omega: \Omega \rightarrow(0, \infty)$ is a measurable weight, we set

$$
L_{\omega}^{1}(\Omega):=\left\{g \in L^{0}(\Omega): \omega g \in L^{1}(\Omega)\right\} \quad \text { endowed with the norm }\|g\|_{L_{\omega}^{1}}:=\|\omega g\|_{L^{1}} .
$$

Our main example will be

$$
\text { the weight in } \mathbb{R}^{d} \text { (or in } Q \text { ) } \quad \kappa(x):=1+|x|^{2} .
$$

In this case, a function $m$ as in (2.2) belongs to $L_{\kappa}^{1}\left(\mathbb{R}^{d}\right)$ if and only if the corresponding measure $\mu=m \mathscr{L}^{d}$ belongs to the space $\mathcal{P}_{2}^{r}\left(\mathbb{R}^{d}\right)$ of absolutely continuous, Borel probability measures with finite quadratic momentum, i.e.

$$
\int_{\mathbb{R}^{d}}|x|^{2} m(x) \mathrm{d} x=\int_{\mathbb{R}^{d}}|x|^{2} \mathrm{~d} \mu(x)<\infty,
$$

so that

$$
\|m\|_{L_{\kappa}^{1}\left(\mathbb{R}^{d}\right)}=\int_{\mathbb{R}^{d}}\left(1+|x|^{2}\right) m(x) \mathrm{d} x=1+\int_{\mathbb{R}^{d}}|x|^{2} \mathrm{~d} \mu(x) \quad \text { for every } \mu=m \mathscr{L}^{d} \in \mathcal{P}_{2}^{r}\left(\mathbb{R}^{d}\right) .
$$

The dual of the space $L_{\omega}^{1}(\Omega)$ can be naturally identified with

$$
L_{1 / \omega}^{\infty}(\Omega):=\left\{h \in L^{0}(\Omega): \omega^{-1} h \in L^{\infty}(\Omega)\right\} \quad \text { endowed with the norm }\|h\|_{L_{1 / \omega}^{\infty}}:=\left\|\omega^{-1} h\right\|_{L^{\infty}} .
$$

In particular, functions $h \in L_{1 / \kappa}^{\infty}\left(\mathbb{R}^{d}\right)$ are naturally in duality with measures $\mu=m \mathscr{L}^{d} \in \mathcal{P}_{2}^{r}\left(\mathbb{R}^{d}\right)$ since $h m \in L^{1}\left(\mathbb{R}^{d}\right)$ so that

$$
\int_{\mathbb{R}^{d}}|h| \mathrm{d} \mu(x)=\int_{\mathbb{R}^{d}}|h| m \mathrm{~d} x \leq\|h\|_{L_{1 / \kappa}^{\infty}}\|m\|_{L_{\kappa}^{1}}<\infty
$$

Eventually we will set

$$
\mathcal{P}_{2, p}^{r}\left(\mathbb{R}^{d}\right):=\left\{\mu=m \mathscr{L}^{d} \in \mathcal{P}_{2}^{r}\left(\mathbb{R}^{d}\right): m \in L^{p}\left(\mathbb{R}^{d}\right)\right\}
$$

A function $G: \mathbb{R}^{d} \times \mathbb{R}^{h} \rightarrow \mathbb{R}$ is a Carathéodory function if

$$
\text { for a.e. } x \in \mathbb{R}^{d} \quad y \mapsto G(x, y) \text { is continuous in } \mathbb{R}^{h} \text {, }
$$

for every $y \in \mathbb{R}^{h} \quad x \mapsto G(x, y)$ is Lebesgue measurable in $\mathbb{R}^{d}$.

If $z: Q \rightarrow \mathbb{R}^{h}$ is a measurable map, we adopt the convention to write $G(x, z)$ for the function $(t, x) \mapsto$ $G(x, z(t, x))$ defined in $Q$. The Carathéodory assumption on $G$ guarantees that such a composition is also measurable.

We say that a real function $f$ defined on some interval $J$ of $\mathbb{R}$ is increasing (resp. strictly increasing) if for every $r_{1}<r_{2}$ in $J$ it holds $f\left(r_{1}\right) \leq f\left(r_{2}\right)$ (resp. $f\left(r_{1}\right)<f\left(r_{2}\right)$ ).

\subsection{Structural assumptions}

We will be mainly concerned with the following first order system

$$
\left\{\begin{aligned}
-\partial_{t} u+H(x, D u) & =f(x, m) & & \text { in } Q, \\
\partial_{t} m-\nabla \cdot\left(m H_{p}(x, D u)\right) & =0 & & \text { in } Q,
\end{aligned}\right.
$$

with initial and final conditions

$$
m(0, \cdot)=m_{0}, m(1, \cdot)=m_{1} \quad \text { in } \mathbb{R}^{d} .
$$

We fix a pair of conjugate exponents $p, q \in(1,+\infty), p^{-1}+q^{-1}=1$ and the weight function $\kappa(x):=1+|x|^{2}$ as in (2.4). The following assumptions hold true throughout the paper. 


\section{Assumptions 2.1}

(H1) $m_{0}, m_{1}$ are nonnegative functions in $L_{\kappa}^{1}\left(\mathbb{R}^{d}\right)$ with equal, normalized, mass:

$$
\int_{\mathbb{R}^{d}} m_{0}(x) \mathrm{d} x=\int_{\mathbb{R}^{d}} m_{1}(x) \mathrm{d} x=1, \quad \int_{\mathbb{R}^{d}}|x|^{2} m_{i}(x) \mathrm{d} x=M_{i}<\infty ; \quad \mu_{i}=m_{i} \mathscr{L}^{d} \in \mathcal{P}_{2}^{r}\left(\mathbb{R}^{d}\right) .
$$

In most part of our analysis, we will also assume that $m_{i} \in L^{p}\left(\mathbb{R}^{d}\right)$.

(H2) $f: \mathbb{R}^{d} \times[0,+\infty) \rightarrow \mathbb{R}$ is a Caratheodory function, increasing with respect to the second variable. There exist a constant $c_{f} \geq 1$ and a nonnegative function $\gamma_{f} \in L^{q}\left(\mathbb{R}^{d}\right)$ such that

$$
\frac{1}{c_{f}^{p}}|m|^{p-1}-\gamma_{f}(x) \leq f(x, m) \leq c_{f}^{p}|m|^{p-1}+\gamma_{f}(x) \quad \text { for every } m \in[0, \infty) \text { and a.e. } x \in \mathbb{R}^{d} .
$$

(H3) The Hamiltonian $H: \mathbb{R}^{d} \times \mathbb{R}^{d} \rightarrow \mathbb{R}$ is a Caratheodory function and it is convex and differentiable with respect to its second variable, with differential which will be denoted by $H_{p}: \mathbb{R}^{d} \times \mathbb{R}^{d} \rightarrow \mathbb{R}^{d}$.

There exist constants $c_{H} \geq 1, c_{H}^{ \pm}>0$ with

$$
\gamma_{H}^{+}(x):=c_{H}^{+}(1+|x|), \quad \gamma_{H}^{-}(x): c_{H}^{-}\left(1+|x|^{2}\right) \quad x \in \mathbb{R}^{d},
$$

such that

$$
\frac{1}{2 c_{H}}|\boldsymbol{p}|^{2}-\gamma_{H}^{-}(x) \leq H(x, \boldsymbol{p}) \leq \frac{c_{H}}{2}|\boldsymbol{p}|^{2}+\gamma_{H}^{+}(x) \quad \text { for every } \boldsymbol{p} \in \mathbb{R}^{d} \text { and a.e. } x \in \mathbb{R}^{d} .
$$

If we define the function $F: \mathbb{R}^{d} \times[0,+\infty) \rightarrow \mathbb{R}$ and its extension $\tilde{F}: \mathbb{R}^{d} \times \mathbb{R} \rightarrow \mathbb{R} \cup\{+\infty\}$ by

$$
F(x, m):=\int_{0}^{m} f(x, \tau) \mathrm{d} \tau, \quad \tilde{F}(x, m):= \begin{cases}F(x, m) & \text { if } m \geq 0 \\ +\infty & \text { otherwise }\end{cases}
$$

then $F$ is (the restriction of a) Carathéodory function and for almost every $x \in \mathbb{R}^{d}$ the map $m \mapsto F(x, m)$ is convex in $\mathbb{R}$ and differentiable in $(0, \infty)$. $F$ is also strictly convex if $f$ is strictly increasing w.r.t. $m$.

Due to Assumption (H2), $F$ satisfies

$$
\frac{1}{p c_{f}^{p}}|m|^{p}-\gamma_{f}(x) m \leq F(x, m) \leq \frac{c_{f}^{p}}{p}|m|^{p}+\gamma_{f}(x) m \quad \text { for every } m \in[0, \infty) \text { and } x \in \mathbb{R}^{d} .
$$

We will denote by $F^{*}: \mathbb{R}^{d} \times \mathbb{R} \rightarrow \mathbb{R}$ the Fenchel conjugate of $F$ with respect to the second variable

$$
F^{*}(x, a):=\sup _{m \in \mathbb{R}}[a m-F(x, m)]=\sup _{m \geq 0}[a m-F(x, m)] .
$$

Starting from (2.15) it is not difficult to check that

$$
\begin{aligned}
& \frac{1}{q c_{f}^{q}}\left(a-\gamma_{f}(x)\right)_{+}^{q} \leq F^{*}(x, a) \leq \frac{c_{f}^{q}}{q}\left(a+\gamma_{f}(x)\right)_{+}^{q} \quad \text { for every } a \in \mathbb{R} \text { and for a.e. } x \in \mathbb{R}^{d}, \\
& a \mapsto F^{*}(x, a) \text { is positive and increasing in } \mathbb{R} \text { for a.e. } x \in \mathbb{R}^{d}, F^{*}(x, a)=0 \text { iff } a \leq f(x, 0) .
\end{aligned}
$$

We will also consider the Lagrangian $L: \mathbb{R}^{d} \times \mathbb{R}^{d} \rightarrow \mathbb{R}$ obtained by evaluating the Fenchel conjugate of $H(x, \boldsymbol{p})$ in the variable $-\boldsymbol{q}$ :

$$
L(x, \boldsymbol{q})=H^{*}(x,-\boldsymbol{q}):=\sup _{\boldsymbol{p} \in \mathbb{R}^{d}}[-\boldsymbol{q} \cdot \boldsymbol{p}-H(x, \boldsymbol{p})] \quad x, \boldsymbol{q} \in \mathbb{R}^{d} .
$$

In particular $L$ is a Carathéodory function, convex with respect to the second variable, and satisfies the growth conditions

$$
\frac{1}{2 c_{H}}|\boldsymbol{q}|^{2}-\gamma_{H}^{+}(x) \leq L(x, \boldsymbol{q}) \leq \frac{c_{H}}{2}|\boldsymbol{q}|^{2}+\gamma_{H}^{-}(x) \quad \text { for every } x, \boldsymbol{q} \in \mathbb{R}^{d} .
$$

Notice that (H2) and (2.15) allow for functions of the form

$$
f(x, m):=a(x) m^{p-1}+V_{f}(x), \quad F(x, m):=\frac{1}{p} a(x) m^{p}+V_{f}(x) m
$$


where $a \in L^{\infty}\left(\mathbb{R}^{d}\right)$ satisfy $\frac{1}{c_{f}^{p}} \leq a(x) \leq c_{f}^{p}$ a.e. in $\mathbb{R}^{d}$ and $V_{f} \in L^{q}\left(\mathbb{R}^{d}\right)$.

Similarly, (H3) and (2.20) allow for Hamiltonians and Lagrangians of the form

$$
\begin{aligned}
H(x, \boldsymbol{p}) & :=\frac{1}{2} \sum_{i, j=1}^{d} g^{i j}(x) p_{i} p_{j}+\sum_{i=1}^{d} z_{i}(x) p_{i}-V_{H}(x), \\
L(x, \boldsymbol{q}) & :=\frac{1}{2} \sum_{i, j=1}^{d} g_{i j}(x)\left(q_{i}-z_{i}(x)\right)\left(q_{j}-z_{j}(x)\right)+V_{H}(x)
\end{aligned}
$$

where $\left(g^{i j}(x)\right)_{i, j}$ are the coefficients of a symmetric elliptic matrix $G(x) \in \mathbb{M}^{d \times d}$ satisfying $\frac{1}{c_{H}} I \leq G(x) \leq c_{H} I$ (in the sense of quadratic forms), $\left(g_{i j}(x)\right)_{i, j}$ are the coefficients of the metric tensor $G^{-1}(x),\left(z_{i}(x)\right)_{i}$ are the components of a measurable and bounded vector field $\boldsymbol{z}: \mathbb{R}^{d} \rightarrow \mathbb{R}^{d}$ and $V_{H}: \mathbb{R}^{d} \rightarrow \mathbb{R}$ is a measurable potential satisfying

$$
-C(1+|x|) \leq V_{H}(x) \leq C\left(1+|x|^{2}\right) \quad \mathscr{L}^{d} \text {-a.e. in } \mathbb{R}^{d} .
$$

\section{Optimal transport distance, displacement interpolation, and continuity equation}

\subsection{Recaps on Optimal Transport and dynamic formulation}

The set $\mathcal{P}_{2}\left(\mathbb{R}^{d}\right)$ of probability measures with finite quadratic moment (2.5) can be naturally endowed with the so-called $L^{2}$-Kantorovich-Rubinstein-Wasserstein distance $W_{2}$. If $\mu_{0}, \mu_{1} \in \mathcal{P}_{2}\left(\mathbb{R}^{d}\right)$, we define

$$
W_{2}^{2}\left(\mu_{0}, \mu_{1}\right):=\min \left\{\int_{\mathbb{R}^{d} \times \mathbb{R}^{d}}\left|x_{0}-x_{1}\right|^{2} \mathrm{~d} \boldsymbol{\mu}\left(x_{0}, x_{1}\right): \boldsymbol{\mu} \in \mathcal{P}\left(\mathbb{R}^{d} \times \mathbb{R}^{d}\right), \pi_{\sharp}^{0} \boldsymbol{\mu}=\mu_{0}, \pi_{\sharp}^{1} \boldsymbol{\mu}=\mu_{1}\right\},
$$

where $\pi^{i}: \mathbb{R}^{d} \times \mathbb{R}^{d} \rightarrow \mathbb{R}^{d}, i=0,1$, stand for the coordinate projections $\pi^{i}\left(x_{0}, x_{1}\right):=x_{i}$.

Recall that for every Borel map $T: X \rightarrow Y$ between two Borel subsets $X, Y$ of some Euclidean space and every Borel measure $\mu$ on $X, T_{\sharp} \mu$ denotes the image measure on $Y$ defined by $T_{\sharp} \mu(A):=\mu\left(T^{-1}(A)\right)$, for every Borel set $A \in Y$.

It is well known that $\left(\mathcal{P}_{2}\left(\mathbb{R}^{d}\right), W_{2}\right)$ is a complete metric space (see e.g. [3, Prop. 7.1.5]) and that a sequence $\left(\mu_{n}\right)_{n \in \mathbb{N}}$ in $\mathcal{P}_{2}\left(\mathbb{R}^{d}\right)$ converges to $\mu$ w.r.t. $W_{2}$ as $n \rightarrow \infty$ if and only if

$$
\lim _{n \rightarrow \infty} \int_{\mathbb{R}^{d}} \varphi(x) \mathrm{d} \mu_{n}(x)=\int_{\mathbb{R}^{d}} \varphi(x) \mathrm{d} \mu(x) \quad \text { for every } \varphi \in C\left(\mathbb{R}^{d}\right) \cap L_{1 / \kappa}^{\infty}\left(\mathbb{R}^{d}\right),
$$

where $\kappa$ is the usual weight $\kappa(x):=1+|x|^{2}$.

A plan $\boldsymbol{\mu}$ attaining the minimum in (3.1) is called optimal. If $\boldsymbol{\mu}$ is an optimal plan, McCann's displacement interpolation [24] [3, Sect. 7.3]

$$
t \mapsto \mu_{t}:=\left((1-t) \pi^{0}+t \pi^{1}\right)_{\sharp} \boldsymbol{\mu}, \quad t \in[0,1],
$$

gives rise to a (mimimal, constant speed) geodesic curve $\left(\mu_{t}\right)_{t \in[0,1]}$ satisfying

$$
W_{2}\left(\mu_{s}, \mu_{t}\right)=|t-s| W_{2}\left(\mu_{0}, \mu_{1}\right) \quad \text { for every } s, t \in[0,1] .
$$

The quadratic momentum is convex along a Wasserstein geodesic:

$$
\int_{\mathbb{R}^{d}}|x|^{2} \mathrm{~d} \mu_{t} \leq(1-t) \int_{\mathbb{R}^{d}}|x|^{2} \mathrm{~d} \mu_{0}+t \int_{\mathbb{R}^{d}}|x|^{2} \mathrm{~d} \mu_{1} .
$$

If $\mu_{i}=m_{i} \mathscr{L}^{d} \in \mathcal{P}_{2, p}^{r}\left(\mathbb{R}^{d}\right)$ then all the measures $\mu_{t}$ given by (3.3) admit a Lebesgue density in $L^{p}\left(\mathbb{R}^{d}\right)$ satisfying the dispacement convexity inequality [24], [3, Thm. 9.3.9]

$$
\mu_{t}=m_{t} \mathscr{L}^{d}, \quad\left\|m_{t}\right\|_{L^{p}\left(\mathbb{R}^{d}\right)}^{p} \leq(1-t)\left\|m_{0}\right\|_{L^{p}\left(\mathbb{R}^{d}\right)}^{p}+t\left\|m_{1}\right\|_{L^{p}\left(\mathbb{R}^{d}\right)}^{p} \quad \text { for every } t \in[0,1] .
$$

\section{Absolutely continuous curves in $\mathcal{P}_{2}\left(\mathbb{R}^{d}\right)$ and the continuity equation}

It is clear from (3.4) that every geodesic is a Lipschitz curve from $[0,1]$ to $\mathcal{P}_{2}\left(\mathbb{R}^{d}\right)$. Given a metric space $\left(Y, d_{Y}\right)$ (our main examples will be $\mathbb{R}^{d}$ and $\mathcal{P}_{2}\left(\mathbb{R}^{d}\right)$ ), we will more generally consider the class $\operatorname{AC}^{2}([a, b] ; Y)$ of absolutely continuous curve with $L^{2}$ metric velocity: they are maps $y:[a, b] \rightarrow Y$ satisfying

$$
d_{Y}(y(s), y(t)) \leq \int_{s}^{t} \rho(r) \mathrm{d} r \quad a \leq s<t \leq b \quad \text { for some } \rho \in L^{2}(a, b) .
$$


Whenever $y \in \mathrm{AC}^{2}([a, b] ; Y)$, the minimal function $\rho$ providing the bound (3.7) is given by the metric velocity

$$
|\dot{y}|(t):=\limsup _{h \rightarrow 0} \frac{d_{Y}(y(t+h), y(t))}{|h|} .
$$

In order to clarify the connection between absolutely continuous curves $\mu:[a, b] \rightarrow \mathcal{P}_{2}\left(\mathbb{R}^{d}\right)$ and the continuity equation, let us first observe that if $\left(\mu_{t}\right)_{t \in[0,1]}$ is a Borel family of probability measures (in particular a continuous curve), the formula

$$
\tilde{\mu}(h):=\int_{0}^{1}\left(\int_{\mathbb{R}^{d}} h(t, x) \mathrm{d} \mu_{t}(x)\right) \mathrm{d} t, \quad h: Q \rightarrow \mathbb{R} \text { bounded and Borel, }
$$

defines a probability measure $\tilde{\mu} \in \mathcal{P}(Q)$ such that

$$
\pi_{\sharp}^{0} \tilde{\mu}=\left.\mathscr{L}^{1}\right|_{[0,1]}, \quad \text { where } \quad \pi^{0}: Q \rightarrow I, \pi^{0}(t, x):=t .
$$

Conversely, any measure $\tilde{\mu} \in \mathcal{P}(Q)$ satisfying (3.10) can be associated to a Borel family $\left(\mu_{t}\right)_{t \in[0,1]}$ satisfying (3.9) by disintegration [3, Sect. 5.3]. We will occasionally identify $\tilde{\mu}$ with $\mu$ when no risk of ambiguity is possible.

If $\mu \in \operatorname{AC}^{2}\left([0,1] ; \mathcal{P}_{2}\left(\mathbb{R}^{d}\right)\right)$ it is possible to find $\left(\left[3\right.\right.$, Thm. 8.3.1]) a Borel vector field $\boldsymbol{v} \in L^{2}\left(Q, \tilde{\mu} ; \mathbb{R}^{d}\right)$, i.e.

$$
\|\boldsymbol{v}\|_{L^{2}\left(Q, \tilde{\mu} ; \mathbb{R}^{d}\right)}^{2}=\int_{Q}|\boldsymbol{v}(t, x)|^{2} \mathrm{~d} \tilde{\mu}(t, x)<+\infty,
$$

such that the pair $(\tilde{\mu}, \boldsymbol{v} \tilde{\mu})$ is a distributional solution to the continuity equation

$$
\partial_{t} \tilde{\mu}+\nabla \cdot(\boldsymbol{v} \tilde{\mu})=0 \quad \text { in } \mathscr{D}^{\prime}(Q)
$$

even in duality with functions in $C_{c}^{1}\left(\mathbb{R}^{d+1}\right)$ :

$$
\int_{Q}\left(\partial_{t} \phi+D \phi \cdot \boldsymbol{v}\right) \mathrm{d} \tilde{\mu}=\int_{\mathbb{R}^{d}} \phi(1, x) \mathrm{d} \mu_{1}(x)-\int_{\mathbb{R}^{d}} \phi(0, x) \mathrm{d} \mu_{0}(x) \quad \text { for every } \phi \in C_{c}^{1}\left(\mathbb{R}^{d+1}\right) .
$$

It is interesting that any choice of vector field $\boldsymbol{v}$ satisfying (3.11) induces a vector measure

$$
\boldsymbol{\nu}=\boldsymbol{v} \tilde{\mu} \ll \tilde{\mu} \text { with finite total variation }|\boldsymbol{\nu}|(Q)=\int_{Q}|\boldsymbol{v}| \mathrm{d} \tilde{\mu} \leq\|\boldsymbol{v}\|_{L^{2}\left(Q, \tilde{\mu} ; \mathbb{R}^{d}\right)},
$$

and a function $\left(\mathscr{L}^{1}\right.$-a.e. defined) $\rho(r):=\left(\int_{\mathbb{R}^{d}}|\boldsymbol{v}(r, x)|^{2} \mathrm{~d} \mu_{r}(x)\right)^{1 / 2}$ in $L^{2}(I)$ satisfying (3.7) for the distance $d_{y}:=W_{2}$ in $\mathcal{P}_{2}\left(\mathbb{R}^{d}\right)$. We can (uniquely) select a minimal one (called the minimal velocity field) such that

$$
\int_{\mathbb{R}^{d}}|\boldsymbol{v}(r, x)|^{2} \mathrm{~d} \mu_{r}(x)=\left\|\dot{\mu}_{t}\right\|_{W_{2}}^{2} \quad \text { for } \mathscr{L}^{1} \text {-a.e. } t \in I .
$$

Conversely if $\tilde{\mu} \in \mathcal{P}_{2}(Q)$ satisfies (3.10) and together with $\boldsymbol{v} \in L^{2}\left(Q, \tilde{\mu} ; \mathbb{R}^{d}\right)$ gives rise to a solution to the continuity equation (3.12) then it is possible to prove [3, Lemma 8.1.2, Theorem 8.3.1] that $\tilde{\mu}$ admits a unique disintegration $\left(\mu_{t}\right)_{t \in[0,1]}$ associated to a curve $\mu \in \mathrm{AC}^{2}\left([0,1] ; \mathcal{P}_{2}\left(\mathbb{R}^{d}\right)\right)$, so that $\mu$ can be considered as the "precise representative" of $\tilde{\mu}$.

Thanks to the previous results, there is a natural identification between curves $\mu \in \mathrm{AC}^{2}\left([0,1] ; \mathcal{P}_{2}\left(\mathbb{R}^{d}\right)\right)$ and solutions $(\tilde{\mu}, \boldsymbol{v} \tilde{\mu})$ of the continuity equation (3.12) for some $\boldsymbol{v} \in L^{2}\left(Q, \tilde{\mu} ; \mathbb{R}^{d}\right)$.

\section{2 $L^{p}$ probability densities with finite action and the Kantorovich-Lebesgue interpolation cost}

In the setting of the mean field system (2.10), it will be more natural to start from a nonnegative density function $m \in L^{1}(Q)$ associated to the measure $m \lambda, \lambda$ being the restriction of $\mathscr{L}^{d+1}$ to $Q$. In order to express in an intrinsic way the regularity hidden in the continuity equation, we will introduce the following definition.

Definition 3.1 (Densities with finite $L^{2}$-action) We say that the probability density (see (2.2) $m \in$ $L_{\kappa}^{1}(Q)$ has finite $L^{2}$-action if there exists a constant $C>0$ such that

$$
-\int_{Q} \partial_{t} \zeta(t, x) m(t, x) \mathrm{d} \lambda \leq C\left(\int_{Q}|D \zeta(t, x)|^{2} m(t, x) \mathrm{d} \lambda\right)^{1 / 2} \quad \text { for every } \zeta \in C_{c}^{1}(Q) \text {. }
$$

We will denote by $\mathrm{A}_{2}(Q)$ the convex subset of $L^{1}(Q)$ of probability densities with finite $L^{2}$-action. 
Notice that if $m$ is a probability density in $L_{\kappa}^{1}(Q)$ and there exists a measurable vector field $\boldsymbol{v}: Q \rightarrow \mathbb{R}^{d}$ such that

$$
\int_{Q}|\boldsymbol{v}|^{2} m \mathrm{~d} \lambda<+\infty, \quad \partial_{t} m+\nabla \cdot(m \boldsymbol{v})=0 \quad \text { in } \mathscr{D}^{\prime}(Q),
$$

then $m \in \mathrm{A}_{2}(Q)$ and we can choose $C:=\left(\int_{Q}|\boldsymbol{v}|^{2} m \mathrm{~d} \lambda\right)^{1 / 2}$ in (3.16). In the next lemma we show that the converse is also true.

Lemma 3.2 (Precise representative and traces of densities with finite $L^{2}$-action) If $m \in \mathrm{A}_{2}(Q)$ then there exists a unique curve $\mu \in \mathrm{AC}^{2}\left([0,1] ; \mathcal{P}_{2}\left(\mathbb{R}^{d}\right)\right)$ such that $m \lambda=\tilde{\mu}$. In particular:

1. The traces of $m$ at $t=0$ and at $t=1$ are well defined probability measures $\mu_{0}, \mu_{1} \in \mathcal{P}_{2}\left(\mathbb{R}^{d}\right)$.

2. There exists a Borel velocity field $\boldsymbol{v}: Q \rightarrow \mathbb{R}^{d}$ such that (3.17) holds, and for every vector field satisfying (3.17) we have (3.13).

3. If moreover $m \in L^{p}(Q)$ then

$$
D_{p}[\mu]:=\left\{t \in[0,1]: \mu_{t} \ll \mathscr{L}^{d}, \quad \frac{\mathrm{d} \mu_{t}}{\mathrm{~d} \mathscr{L}^{d}} \in L^{p}\left(\mathbb{R}^{d}\right)\right\}
$$

is a dense $F_{\sigma}$ subset in $[0,1]$ (a countable union of closed sets) of full measure.

Proof. We consider the measure $\tilde{\mu}=m \lambda$ and we introduce the linear subspace $V:=\left\{D \zeta: \zeta \in C_{c}^{1}(Q)\right\}$ in $L^{2}\left(Q, \tilde{\mu} ; \mathbb{R}^{d}\right)$. Since the linear map $L: \zeta \mapsto-\int_{Q} \partial_{t} \zeta m \mathrm{~d} \lambda$ defines a bounded functional in $V$, it admits a continuous extension to $L^{2}\left(Q, \tilde{\mu} ; \mathbb{R}^{d}\right)$ which can be represented by a vector field $\boldsymbol{v} \in L^{2}\left(Q, \tilde{\mu} ; \mathbb{R}^{d}\right)$ by $\operatorname{Riesz}$ Theorem:

$$
L(\zeta)=-\int_{Q} \partial_{t} \zeta m \mathrm{~d} \lambda=\int_{Q} D \zeta \cdot \boldsymbol{v} m \mathrm{~d} \lambda \quad \text { for every } \zeta \in C_{c}^{1}(Q) .
$$

We thus find that $(\tilde{\mu}, \boldsymbol{v} \tilde{\mu})$ satisfies the continuity equation (3.12) so that $\tilde{\mu}$ admits a precise representative $\mu \in \mathrm{AC}^{2}\left([0,1] ; \mathcal{P}_{2}\left(\mathbb{R}^{d}\right)\right)$ according to the above considerations.

Finally, if $m \in L^{p}(Q)$ then Fubini's theorem yields

$$
\int_{Q} m^{p} \mathrm{~d} \lambda=\int_{0}^{1}\left(\int_{\mathbb{R}^{d}} m^{p}(t, x) \mathrm{d} x\right) \mathrm{d} t<+\infty, \quad \int_{\mathbb{R}^{d}} m^{p}(t, x) \mathrm{d} x<+\infty, \quad \text { for } \mathscr{L}^{1} \text {-a.e. } t \in(0,1),
$$

so that $D_{p}[\mu]$ is of full measure in $[0,1]$ (in particular, it is dense). $D_{p}[\mu]$ can also be considered as the finiteness domain of the $p$-entropy functional

$$
\mathscr{U}_{p}[\mu]:= \begin{cases}\int_{\mathbb{R}^{d}}(m(x))^{p} \mathrm{~d} x & \text { if } \mu=m \mathscr{L}^{d} \ll \mathscr{L}^{d} \\ +\infty & \text { otherwise }\end{cases}
$$

which is lower semicontinuous w.r.t. weak convergence of measures. $D_{p}[\mu]$ is therefore an $F_{\sigma}$ subset of $[0,1]$.

Notice that if $m \in \mathrm{A}_{2}(Q)$ then $m(t, \cdot)$ is a probability density for $\mathscr{L}^{1}$-a.e. $t \in(0,1)$. We conclude this discussion with two definitions which will play a crucial role in Sections 5.1 and 6 .

Definition 3.3 ( $L^{p}$ solutions to the continuity equation with $L^{2}$-velocity)

Let $p \in(1,+\infty]$. We say that a pair $(m, \boldsymbol{v})$ belongs to the set $\mathrm{CE}_{2, p}(Q)$ if

(1) $m \in L_{\kappa}^{1}(Q) \cap L^{p}(Q)$ is a probability density and $\boldsymbol{v} \in L^{2}\left(Q, \tilde{\mu} ; \mathbb{R}^{d}\right)$ where $\tilde{\mu}=m \lambda$.

(2) The pair $(m, \boldsymbol{v})$ is a solution to the continuity equation (3.17).

In particular $m \in \mathrm{A}_{2}(Q)$ and admits a continuous representative $\mu \in \mathrm{AC}^{2}\left([0,1] ; \mathcal{P}_{2}\left(\mathbb{R}^{d}\right)\right)$ according to Lemma 3.2. Whenever $\mu_{0}, \mu_{1} \in \mathcal{P}_{2}\left(\mathbb{R}^{d}\right)$ are given, we will also set

$$
\mathrm{CE}_{2, p}\left(Q ; \mu_{0}, \mu_{1}\right):=\left\{(m, \boldsymbol{v}) \in \mathrm{CE}_{2, p}(Q): \mu_{\left.\right|_{t=0}}=\mu_{0},\left.\mu\right|_{t=1}=\mu_{1}\right\}
$$

The class $\mathrm{CE}_{2, p}(Q)$ naturally induces a dynamic transport cost between probability measures, which results from the interaction of the Kantorovich action and the $L^{p}$-penalization of the densities. 
Definition 3.4 (The Kantorovich-Lebesgue $K L_{2, p}$ cost) Let $p \in(1,+\infty)$. For every $\mu_{0}, \mu_{1} \in \mathcal{P}_{2}\left(\mathbb{R}^{d}\right)$ and every parameter $a>0$ we set

$$
K L_{2, p}^{(a)}\left(\mu_{0}, \mu_{1}\right):=\inf \left\{\int_{Q}\left(\frac{a}{2}|\boldsymbol{v}|^{2} m+\frac{1}{2 a}\left(m+m^{p}\right)\right) \mathrm{d} \lambda:(m, \boldsymbol{v}) \in \mathrm{CE}_{2, p}\left(Q ; \mu_{0}, \mu_{1}\right)\right\},
$$

with the usual convention $K L_{2, p}^{(a)}\left(\mu_{0}, \mu_{1}\right)=+\infty$ if $\mathrm{CE}_{2, p}\left(Q ; \mu_{0}, \mu_{1}\right)$ is empty. When $a=1$ we will just write $K L_{2, p}\left(\mu_{0}, \mu_{1}\right)$. The induced Kantorovich-Lebesgue distance can be defined as

$$
d_{K L_{2, p}}\left(\mu_{0}, \mu_{1}\right):=\inf _{a>0} K L_{2, p}^{(a)}\left(\mu_{0}, \mu_{1}\right)
$$

It is obvious that

$$
K L_{2, p}^{(a)}\left(\mu_{0}, \mu_{1}\right) \leq \max \left(\frac{a}{b}, \frac{b}{a}\right) K L_{2, p}^{(b)}\left(\mu_{0}, \mu_{1}\right) \quad \text { for every } a, b>0 .
$$

The rescaled costs $K L_{2, p}^{(a)}$ just correspond to the cost $K L_{2, p}$ but for a continuity equation in the dilated cylinder $(0, a) \times \mathbb{R}^{d}$ :

$$
K L_{2, p}^{(a)}\left(\mu_{0}, \mu_{1}\right):=\inf \left\{\int_{0}^{a} \int_{\mathbb{R}^{d}}\left(\frac{1}{2}|\boldsymbol{v}|^{2} m+\frac{1}{2}\left(m+m^{p}\right)\right) \mathrm{d} \lambda:(m, \boldsymbol{v}) \in \mathrm{CE}_{2, p}\left((0, a) \times \mathbb{R}^{d} ; \mu_{0}, \mu_{1}\right)\right\} .
$$

By a standard rescaling argument (see e.g. [29, Lemma 2.2 and A.5]) we obtain

$$
d_{K L_{2, p}}\left(\mu_{0}, \mu_{1}\right)=\inf \left\{\int_{0}^{1}\left(1+\left\|m_{t}\right\|_{L^{p}\left(\mathbb{R}^{d}\right)}^{p}\right)^{1 / 2}\left(\int_{\mathbb{R}^{d}}\left|\boldsymbol{v}_{t}\right|^{2} m_{t} \mathrm{~d} x\right)^{1 / 2} \mathrm{~d} t:(m, \boldsymbol{v}) \in \mathrm{CE}_{2, p}\left(Q ; \mu_{0}, \mu_{1}\right)\right\},
$$

so that $d_{K L_{2, p}} \geq W_{2}$; it is not difficult to show that the cost $K L_{2, p}$ is finite if and only if $d_{K L_{2, p}}$ is finite.

Here we do not aim at characterizing the class of measures for which $K L_{2, p}$ is finite, we will just point out two important cases. The first one consists in probability measures with $L^{p}$ densities $\mu \in \mathcal{P}_{2, p}^{r}\left(\mathbb{R}^{d}\right)$, which can also be identified with the subset of probability densities in $L^{p} \cap L_{\kappa}^{1}\left(\mathbb{R}^{d}\right)$.

Lemma 3.5 If $\mu_{i}=m_{i} \mathscr{L}^{d} \in \mathcal{P}_{2, p}^{r}\left(\mathbb{R}^{d}\right), i=1,2$, then $\mathrm{CE}_{2, p}\left(Q ; \mu_{0}, \mu_{1}\right)$ is not empty and

$$
K L_{2, p}^{(a)}\left(\mu_{0}, \mu_{1}\right) \leq \frac{1}{2 a}+\int_{\mathbb{R}^{d}}\left(a|x|^{2}\left(m_{0}+m_{1}\right)+\frac{1}{4 a}\left(m_{0}^{p}+m_{1}^{p}\right)\right) \mathrm{d} x .
$$

Proof. It is sufficient to choose the McCann's displacement interpolation (3.3), yielding

$$
\int_{Q}|\boldsymbol{v}|^{2} m \mathrm{~d} \lambda=W_{2}^{2}\left(\mu_{0}, \mu_{1}\right) \leq 2 \int_{\mathbb{R}^{d}}|x|^{2} \mathrm{~d} \mu_{0}+2 \int_{\mathbb{R}^{d}}|x|^{2} \mathrm{~d} \mu_{1}
$$

and

$$
\int_{Q} m^{p} \mathrm{~d} \lambda \leq \frac{1}{2}\left(\left\|m_{0}\right\|_{L^{p}\left(\mathbb{R}^{d}\right)}^{p}+\left\|m_{1}\right\|_{L^{p}\left(\mathbb{R}^{d}\right)}^{p}\right) .
$$

The above Lemma shows that the $d_{K L_{2, p}}$ is a distance on the set $\mathcal{P}_{2, p}^{r}\left(\mathbb{R}^{d}\right)$. We can denote by $\mathcal{P}_{2, p}\left(\mathbb{R}^{d}\right)$ its completion w.r.t. $d_{K L_{2, p}}$. The next Lemma will show that this set can be considerably larger than $\mathcal{P}_{2, p}^{r}\left(\mathbb{R}^{d}\right)$.

Lemma 3.6 $\mathcal{P}_{2, p}\left(\mathbb{R}^{d}\right)$ can be identified with the subset of $\mathcal{P}_{2}\left(\mathbb{R}^{d}\right)$ of measures at finite distance from $\mathcal{P}_{2, p}^{r}\left(\mathbb{R}^{d}\right)$ :

$$
\mathcal{P}_{2, p}\left(\mathbb{R}^{d}\right)=\left\{\mu \in \mathcal{P}_{2}\left(\mathbb{R}^{d}\right): \mathrm{CE}_{2, p}\left(Q ; \mu, \mu^{\prime}\right) \text { is not empty for some } \mu^{\prime} \in \mathcal{P}_{2, p}^{r}\left(\mathbb{R}^{d}\right)\right\} .
$$

If $p<1+2 / d$ then $\mathcal{P}_{2, p}\left(\mathbb{R}^{d}\right)=\mathcal{P}_{2}\left(\mathbb{R}^{d}\right)$ so that $K L_{2, p}\left(\mu_{0}, \mu_{1}\right)<\infty$ for every pair $\mu_{0}, \mu_{1} \in \mathcal{P}_{2}\left(\mathbb{R}^{d}\right)$.

If $p \geq 1+2 / d$ then $\mathcal{P}_{2, p}\left(\mathbb{R}^{d}\right) \supset \mathcal{P}_{2, p_{\star}}^{r}\left(\mathbb{R}^{d}\right)$ with $p_{\star}>p /(1+2 / d)$, so that if $\mu_{i}=m_{i} \mathscr{L}^{d} \in \mathcal{P}_{2}^{r}\left(\mathbb{R}^{d}\right)$ for some $m_{i} \in L^{p_{\star}}\left(\mathbb{R}^{d}\right)$ then $K L_{2, p}\left(\mu_{0}, \mu_{1}\right)<\infty$.

Proof. $\quad$ Since $d_{K L_{2, p}} \geq W_{2}$ and $\mathcal{P}_{2}\left(\mathbb{R}^{d}\right)$ is complete, it is easy to check that $\mathcal{P}_{2, p}\left(\mathbb{R}^{d}\right) \subset \mathcal{P}_{2}\left(\mathbb{R}^{d}\right)$. It is obvious that a measure $\mu$ in the completion $\mathcal{P}_{2, p}\left(\mathbb{R}^{d}\right)$ can be connected to measures in $\mathcal{P}_{2, p}^{r}\left(\mathbb{R}^{d}\right)$ with finite cost; conversely, if there exists $\mu^{\prime}=m^{\prime} \mathscr{L}^{d} \in \mathcal{P}_{2, p}^{r}\left(\mathbb{R}^{d}\right)$ with $(m, \boldsymbol{v}) \in \mathrm{CE}_{2, p}\left(Q ; \mu, \mu^{\prime}\right)$ then $m_{t} \in \mathcal{P}_{2, p}^{r}\left(\mathbb{R}^{d}\right)$ for all $t \in D_{p}[\mu]$, in particular there exists a decreasing sequence $t_{n} \downarrow 0$ such that $m_{t_{n}} \in \mathcal{P}_{2, p}^{r}\left(\mathbb{R}^{d}\right)$ and we have

$$
d_{K L_{2, p}}\left(\mu, m_{t_{n}}\right) \leq K L_{2, p}^{t_{n}}\left(\mu, m_{t_{n}}\right) \leq \frac{1}{2} t_{n}+\int_{0}^{t_{n}} \int_{\mathbb{R}^{d}}\left(\frac{1}{2}|\boldsymbol{v}|^{2} m+m^{p}\right) \mathrm{d} x \mathrm{~d} t \rightarrow 0 \text { as } n \rightarrow \infty
$$


so that $\mu$ belongs to the closure of $\mathcal{P}_{2, p}^{r}\left(\mathbb{R}^{d}\right)$ with respect to $d_{K L_{2, p}}$.

Let us now check the last two statements, first considering the case $p<1+2 / d$. By the above argument, it is sufficient to show that any $\mu_{0} \in \mathcal{P}_{2}\left(\mathbb{R}^{d}\right)$ can be connected to a measure $\mu_{1} \in \mathcal{P}_{2, p}^{r}\left(\mathbb{R}^{d}\right)$ by a path $(\mu, \boldsymbol{v}) \in \mathrm{CE}_{2, p}\left(Q ; \mu_{0}, \mu_{1}\right)$.

In order to find $\mu$ we introduce the Heat semigroup $\left(S_{t}\right)_{t \geq 0}$ in $\mathbb{R}^{d}$

$$
S_{t} \mu=\mu * g_{t}, \quad g_{t}(x):=\frac{1}{(4 \pi t)^{d / 2}} \mathrm{e}^{-|x|^{2} / 4 t},
$$

and set $\mu_{t}=m_{t} \mathscr{L}^{d}:=S_{t} \mu_{0}$. It is well known that the $L^{p}$ norm of $m_{t}$ obeys the estimate

$$
\left\|m_{t}\right\|_{L^{p}\left(\mathbb{R}^{d}\right)} \leq C_{p} \frac{1}{t^{(1-1 / p) d / 2}} ;
$$

morever, since $m$ satisfies the Heat equation

$$
\partial_{t} m-\Delta m=0 \quad \text { in }(0, \infty) \times \mathbb{R}^{d}
$$

we see that

$$
\partial_{t} m+\nabla \cdot(m \boldsymbol{v})=0 \quad \text { with } \quad \boldsymbol{v}=-D m / m
$$

and the metric velocity of $m$ w.r.t. the Wasserstein distance at time $t>0$ coincides with the Fisher information

$$
\left|\dot{\mu}_{t}\right|_{W_{2}}^{2}=\int_{\mathbb{R}^{d}}|\boldsymbol{v}(t, x)|^{2} m(t, x) \mathrm{d} x=\int_{\mathbb{R}^{d}} \frac{|D m(t, x)|^{2}}{m(t, x)} \mathrm{d} x .
$$

The convexity of the integrand $(x, \boldsymbol{y}) \mapsto|\boldsymbol{y}|^{2} / x$ in $(0, \infty) \times \mathbb{R}^{d}$ and Jensen's inequality yield (see [3, Lemma 8.1.10])

$$
\int_{\mathbb{R}^{d}} \frac{|D m(t, x)|^{2}}{m(t, x)} \mathrm{d} x \leq \int_{\mathbb{R}^{d}} \frac{\left|D g_{t}(x)\right|^{2}}{g_{t}(x)} \mathrm{d} x=\frac{d}{8 \pi t} .
$$

We deduce the upper bound

$$
\left(1+\|\left. m_{t}\right|_{L^{p}\left(\mathbb{R}^{d}\right)} ^{p}\right)^{1 / 2}\left|\dot{\mu}_{t}\right|_{W_{2}} \leq C t^{-\gamma}, \quad t \in(0,1), \gamma:=\frac{1}{2}+\frac{d}{4}(p-1),
$$

which is integrable in $(0,1)$ if $p<1+d / 2$.

When $\mu_{0}=m_{0} \mathscr{L}^{d}$ with $m_{0} \in L^{p_{\star}}\left(\mathbb{R}^{d}\right)$, we use the same argument replacing (3.30) with

$$
\left\|m_{t}\right\|_{L^{p}\left(\mathbb{R}^{d}\right)} \leq C_{p_{\star}, p} \frac{1}{t^{\left(1 / p_{\star}-1 / p\right) d / 2}}
$$

and thus obtaining an estimate analogous to (3.34) with the exponent $\gamma=\frac{1}{2}+\frac{p d}{4}\left(1 / p_{\star}-1 / p\right)$. The integrability condition near 0 then yields the condition $p_{\star}>p /(1+2 / d)$.

\section{Convergence in measure, increasing functions, weighted $L^{p}$ spaces and anisotropic convolution}

\subsection{The space $L^{0}(\Omega ; \mathfrak{m})$ and the convergence in measure}

Let $\Omega$ be a Polish topological space with its Borel $\sigma$-algebra $\mathscr{B}$ and a $\sigma$-finite Borel measure $\mathfrak{m}$. Since $\mathfrak{m}$ is $\sigma$-finite, we can find a

$$
\text { l.s.c. density function } \rho: \Omega \rightarrow(0,1] \text { such that } \varrho:=\rho \mathfrak{m} \in \mathcal{P}(\Omega) \text {. }
$$

We denoted by $L^{0}(\Omega, \mathfrak{m})$ the space of (equivalent classes of) $\mathfrak{m}$-measurable functions $u: \Omega \rightarrow \mathbb{R} . L^{0}(\Omega, \mathfrak{m})$ is endowed with the topology of the convergence in measure (on every measurable set of finite measure): recall that a sequence $\left(u_{n}\right)_{n \in \mathbb{N}}$ in $L^{0}(\Omega, \mathfrak{m})$ converges to $u \in L^{0}(\Omega, \mathfrak{m})$ in measure if

$$
\text { for every } \varepsilon>0, F \subset \mathscr{B} \text { with } \mathfrak{m}(F)<\infty: \quad \lim _{n \uparrow+\infty} \mathfrak{m}\left(\left\{x \in F:\left|u_{n}(x)-u(x)\right| \geq \varepsilon\right\}\right)=0 .
$$

It is well known (see also Lemma A.2) that this convergence is equivalent to the convergence in measure w.r.t. $\varrho$ and it is metrizable, e.g. by the distance

$$
d(u, v):=\int_{\Omega}(|u(x)-v(x)| \wedge 1) \mathrm{d} \varrho(x) .
$$


If we want to include also functions in $L^{0}(\Omega, \mathfrak{m} ; \overline{\mathbb{R}})$ with values in the extended real line $\overline{\mathbb{R}}=[-\infty,+\infty]$, we can observe that every increasing homeomorphism $\zeta: \overline{\mathbb{R}} \rightarrow[-1 / 2,1 / 2]\left(\right.$ e.g. $\zeta(x):=\frac{x}{2 \sqrt{\left(1+x^{2}\right)}}$ with $\zeta( \pm \infty):= \pm 1 / 2)$ induces a bijection with the set

$$
L^{0}(\Omega, \mathfrak{m} ;[-1 / 2,1 / 2]):=\left\{f \in L^{0}(\Omega, \mathfrak{m}): f(x) \in[-1 / 2,1 / 2] \text { for } \mathfrak{m} \text {-a.e. } x \in \Omega\right\}
$$

via the composition map $f \mapsto \zeta \circ f$. This correspondence and the last statement of Lemma A.2 justifies the following definition.

Definition 4.1 We say that a sequence $f_{n} \in L^{0}(\Omega, \mathfrak{m} ; \overline{\mathbb{R}}), n \in \mathbb{N}$, converges in measure to $f \in L^{0}(\Omega, \mathfrak{m} ; \overline{\mathbb{R}})$ as $n \rightarrow \infty$ if $\zeta \circ f_{n} \rightarrow \zeta \circ f$ in measure in $L^{0}(\Omega, \mathfrak{m})$, or, equivalently, in any $L^{p}(\Omega ; \varrho), 1<p<\infty$.

Let us notice that the above definition is independent on the choice of the map $\zeta$, of $\varrho$ and of $p$. We also notice that when $\Omega$ is an open subset of $\mathbb{R}^{d}$, convergence in $L_{\text {loc }}^{1}(\Omega)$ implies convergence in measure, thanks to characterization (b) of Lemma A.2.

\subsection{Properties of increasing functions}

In this section we will deal with (class of) measurable functions $u \in L^{0}(I \times \Omega, \tilde{\mathfrak{m}}), \tilde{\mathfrak{m}}:=\mathscr{L}^{1} \otimes \mathfrak{m}$, which are increasing w.r.t. time. In principle, they could be characterized by three different properties:

(i) an integral inequality against $C^{1}$ function w.r.t. time (a distributional inequality $-\partial_{t} u \leq 0$ in $\mathscr{D}^{\prime}(Q)$ when $\Omega=\mathbb{R}^{d}$ ): this is the most natural way to write a condition invariant w.r.t. modifications of $u$ in a $\tilde{\mathfrak{m}}$-negligible set.

(ii) $u$ admits a Borel representative $\mathrm{u}: I \times \Omega \rightarrow \mathbb{R}$ such that $\mathrm{u}(s, \cdot) \leq \mathrm{u}(t, \cdot) \mathfrak{m}$-a.e. in $\Omega$ for every $0<s<$ $t<1$.

(iii) there exists a Borel representative $\mathrm{u}: I \times \Omega \rightarrow \mathbb{R}$ and a $\mathfrak{m}$-negligible subset $N \subset \Omega$ such that $\mathrm{u}(s, x) \leq$ $\mathrm{u}(t, x)$ for every $x \in \Omega \backslash N$ and $0<s<t<1$ (differently from (ii), the exceptional set $N$ does not depend on $s$ and $t$ ).

We will discuss the (well known) equivalence between (i), (ii), (iii), by supplementing the analysis with the enucleation of a (suitable) right continuous representative, the existence of the traces at $t=0$ and $t=1$ in $L^{0}\left(\mathbb{R}^{d} ; \overline{\mathbb{R}}\right)$, the approximation by time convolution, and the natural variant corresponding to the differential inequality $-\partial_{t} u \leq \beta$.

Let us first discuss the case (ii): notice that $L^{0}(\Omega, \mathfrak{m})$ can be endowed with a partial order by

$$
u \leq v \quad \Leftrightarrow \quad u(x) \leq v(x) \quad \text { for } \mathfrak{m} \text {-a.e. } x \in \Omega,
$$

so that we can consider increasing maps $t \mapsto u_{t} \in L^{0}(\Omega, \mathfrak{m})$ defined in some subset $J$ of the real line: they satisfy $u_{s} \leq u_{t}$ for every $s, t \in J$ with $s<t$. We will say that $u$ is right continuous if for every right accumulation point $t$ of $J \lim _{s \downarrow t} u_{s}=u_{t}$ in $L^{0}(\Omega, \mathfrak{m})$.

\section{Lemma 4.2}

(1) Let $\left(u_{n}\right)_{n \in \mathbb{N}}$ be an increasing sequence in $L^{0}(\Omega, \mathfrak{m})$. Then there exists a unique $u \in L^{0}(\Omega, \mathfrak{m} ; \overline{\mathbb{R}})$ such that $u_{n} \rightarrow u$ in measure as $n \rightarrow \infty$.

(2) Let $t \mapsto u_{t}$ be an increasing map from $D$ to $L^{0}(\Omega, \mathfrak{m})$, where $D \subset I$ is a dense set. Then there exist unique maps $u^{-}: I \rightarrow L^{0}(\Omega, \mathfrak{m})$ (resp. $\left.u^{+}\right)$and $u_{1}^{-} \in L^{0}(\Omega, \mathfrak{m} ; \mathbb{R} \cup\{+\infty\})\left(\right.$ resp. $\left.u_{0}^{+} \in L^{0}(\Omega, \mathfrak{m} ; \mathbb{R} \cup\{-\infty\})\right)$ such that $\lim _{s \in D, s \uparrow t} u_{s}=u_{t}^{-}$in measure for every $t \in(0,1]$ (resp. $\lim _{s \in D, \downarrow t} u_{s}=u_{t}^{+}$for every $t \in[0,1)$ ).

(3) If $D \ni t \mapsto u_{t}, D \subset I$ dense, is increasing then for every $t \in D$ it holds $u_{t}^{-} \leq u_{t} \leq u_{t}^{+}$and there exists an at most countable set $J_{u} \subset D$ such that $u_{t}^{-}=u_{t}=u_{t}^{+}$for every $t \in D \backslash J_{u}$.

(4) If $t \mapsto u_{t}$ is increasing then $t \mapsto u_{t}^{+}$is increasing and right continuous with values in $L^{0}(\Omega, \mathfrak{m}) . u_{t}=u_{t}^{+}$ for every $t \in I$ if and only if $u$ is right continuous.

Proof. We will use the notation of Section 4.1 in particular the probability measure $\varrho$ and the homemorphism $\zeta$.

(1) We can select a common $\mathfrak{m}$-negligible set $N \subset \Omega$ such that $u_{n}$ are defined in $\Omega \backslash N$ and

$$
u_{m}(x) \leq u_{n}(x) \quad \text { for every } x \in \Omega \backslash N \quad \text { if } m<n .
$$


Now define $u(x):=\lim _{n \uparrow \infty} u_{n}(x)=\sup _{n \in \mathbb{N}} u_{n}(x)$ for every $x \in \Omega \backslash N$. If we apply the Lebesgue dominated convergence theorem in $L^{1}(\Omega ; \varrho)$ to the sequence $\zeta \circ u_{n}$, which is pointwise a.e. converging towards $\zeta \circ u$ as $n \rightarrow+\infty$, we easily obtain that $u_{n} \rightarrow u$ in measure.

(2) It is not restrictive to consider the case $u_{t}^{-}$and fix $t=1$. We first choose a sequence $D \ni t_{n} \uparrow 1$ and define $u_{1}^{-}$applying claim (1). By monotonicity, if $t \geq t_{n}$ it holds

$$
\int_{\Omega}\left|\zeta\left(u_{1}^{-}(x)\right)-\zeta\left(u_{t}(x)\right)\right| \mathrm{d} \varrho(x)=\int_{\Omega}\left[\zeta\left(u_{1}^{-}(x)\right)-\zeta\left(u_{t}(x)\right)\right] \mathrm{d} \varrho(x) \leq \int_{\Omega}\left[\zeta\left(u_{1}^{-}(x)\right)-\zeta\left(u_{t_{n}}(x)\right)\right] \mathrm{d} \varrho(x),
$$

so that $\lim _{t \uparrow 1} \int_{\Omega}\left|\zeta\left(u_{1}^{-}(x)\right)-\zeta\left(u_{t}(x)\right)\right| d \varrho(x)=0$, yielding the convergence in measure.

(3) By monotonicity it is immediate to check that for every $r<s<t$ in $D$

$$
u_{r} \leq u_{s}^{-} \leq u_{s} \leq u_{s}^{+} \leq u_{t}
$$

The function $w(t):=\int_{\Omega} \zeta\left(u_{t}(x)\right) \mathrm{d} \varrho(x)$ is increasing with $w^{ \pm}(t)=\int_{\Omega} \zeta\left(u_{t}^{ \pm}(x)\right) \mathrm{d} \varrho(x)$. We know that the set $J_{w}=\left\{t: w^{+}(t)>w^{-}(t)\right\}$ is at most countable. On the other hand

$$
w^{+}(t)=\int_{\Omega} \zeta\left(u_{t}^{+}(x)\right) d \varrho(x)=\int_{\Omega} \zeta\left(u_{t}^{-}(x)\right) d \varrho(x)=w^{-}(t), \quad \forall t \notin J_{w} .
$$

Since $u_{t}^{-} \leq u_{t}^{+}$and $\zeta$ is strictly monotone, we deduce $u_{t}^{-}=u_{t}^{+}$for every $t \in I \backslash J_{w}$ and we conclude.

(4) It follows immediately by the previous claims.

Let us now consider the case of functions defined in $\Omega_{I}:=I \times \Omega$ endowed with the product measure $\tilde{\mathfrak{m}}:=\mathscr{L}^{1} \otimes \mathfrak{m}$. We will call $B_{f}(\Omega)$ the space of bounded Borel maps $\varphi: \Omega \rightarrow \mathbb{R}$ vanishing outside a set of finite measure, i.e. $\mathfrak{m}\{\varphi \neq 0\}<\infty$. If $\beta \in L^{0}(I \times \Omega, \tilde{\mathfrak{m}})$ satisfies the property

$$
\int_{(a, b) \times F}|\beta(t, x)| \operatorname{dm}(t, x)<\infty \quad \text { for every } 0<a<b<1, \quad F \in \mathscr{B}, \mathfrak{m}(F)<\infty,
$$

we may select a Borel representative $\tilde{\beta}$ of $\beta$ such that

$$
\tilde{\beta}(\cdot, x) \in L^{1}(a, b) \text { for every } x \in \Omega, 0<a<b<1 \text {, and } B(t, x):=\int_{1 / 2}^{t} \tilde{\beta}(s, x) \mathrm{d} s,
$$

is absolutely continuous w.r.t. $t \in I$ for every $x \in \Omega$.

We will make extensively use of the standard regularization technique by convolution. Thus we fix a family $h_{\tau}, \tau>0$, of convolution kernels

$$
h \in C_{c}^{\infty}(\mathbb{R}), \quad h \geq 0, \quad \operatorname{supp} h \subset(-1,0), \quad \int_{\mathbb{R}} h \mathrm{~d} t=1, \quad h_{\tau}(t):=\tau^{-1} h(t / \tau) .
$$

If $u$ is a Borel everywhere defined representative, the set $\Omega_{1}(u):=\left\{x \in \Omega: u(\cdot, x) \in L^{1}(a, b)\right.$ for every $0<$ $a<b<1\}$ is Borel and we can define the function

$$
u_{\tau}(t, x):= \begin{cases}\int_{\mathbb{R}} h_{\tau}(t-s) u(s, x) \mathrm{d} s & \text { if } x \in \Omega_{1}(u) \\ 0 & \text { otherwise }\end{cases}
$$

which is Borel, it is $C^{\infty}(0,1-\tau)$ w.r.t. $t$ for every $x$, and satisfies $u_{\tau}=u_{\tau}^{\prime} \tilde{\mathfrak{m}}$-a.e. whenever $u=u^{\prime} \tilde{\mathfrak{m}}$-a.e. Finally, we will consider pairs $(\zeta, Z)$ in the sets

$$
\mathscr{Z}:=\left\{(\zeta, Z): \zeta \in C_{b}^{0}(\mathbb{R}), \zeta \geq 0, Z(r)=\int_{0}^{r} \zeta(s) \mathrm{d} s\right\}, \quad \mathscr{Z}_{c}:=\left\{(\zeta, Z) \in \mathscr{Z}: \zeta \in C_{c}^{0}(\mathbb{R})\right\} .
$$

Lemma 4.3 Let $u, \beta \in L^{0}(I \times \Omega)$ with $\tilde{\beta}$ and $B$ as in (4.8) and (4.9). The following properties are equivalent:

(i) For every nonnegative $\eta \in C_{c}^{1}(I)$ and $\varphi \in B_{f}(\Omega)$, and every pair $(\zeta, Z) \in \mathscr{Z}_{c}$

$$
\int_{\Omega_{I}} \eta^{\prime}(t) \varphi(x) Z(u(t, x)) \mathrm{d} \tilde{\mathfrak{m}}(t, x) \leq \int_{\Omega_{I}} \eta(t) \varphi(x) \zeta(u(t, x)) \beta(t, x) \mathrm{d} \tilde{\mathfrak{m}}(t, x) .
$$

(ii) There exists a Borel representative $\tilde{u}$ and a Borel set $D$ of full $\mathscr{L}^{1}$-measure in $(0,1)$ such that

$$
\tilde{u}(s, \cdot)-B(s, \cdot) \leq \tilde{u}(t, \cdot)-B(t, \cdot) \quad \mathfrak{m} \text {-a.e. in } \Omega \text { for every } s, t \in D, s<t \text {. }
$$


(iii) There exists a Borel representative u such that $\mathrm{u}(s, x)-B(s, x) \leq \mathrm{u}(t, x)-B(t, x)$ for every $x \in \Omega$ and $s, t \in I, s<t$.

Moreover, if one of the above conditions holds, then

(I.1) $\mathrm{u}$ can be chosen so that $t \mapsto \mathrm{u}(t, x)$ is right continuous for every $x \in \Omega$ (this will be called a precise representative of $u)$; if $\mathrm{u}_{1}, \mathrm{u}_{2}$ are two precise representatives of $u$ then for $\mathfrak{m}-a . e . x \in \Omega$ they satisfy $\mathrm{u}_{1}(\cdot, x)=\mathrm{u}_{2}(\cdot, x)$ everywhere on I. In particular $\mathrm{u}(\cdot, x)$ do not depend on $\beta$, up to $\mathfrak{m}$-negligible sets.

(I.2) If $\mathrm{u}^{-}(t, x):=\lim _{s \uparrow t} \mathrm{u}(s, x), x \in \Omega$, we have

$$
\mathrm{u}^{-}(t, \cdot) \leq \tilde{u}(t, \cdot) \leq \mathrm{u}(t, \cdot) \quad \mathfrak{m} \text {-a.e. in } \Omega, \text { for every } t \in D
$$

and equality holds in (4.14) with at most countable exceptions.

(I.3) $\Omega_{1}(\mathrm{u})=\Omega$ and $\mathrm{u}(t, x)=\lim _{\tau \downarrow 0} \mathrm{u}_{\tau}(t, x)$ for every $t \in I, x \in \Omega$.

(I.4) If $u \in L^{1}((a, b) \times \Omega, \tilde{\mathfrak{m}})$ for every $0<a<b<1$, then (4.12) holds if and only if it is satisfied with the choice $Z(u) \equiv u, \zeta(u) \equiv 1$.

(I.5) If $u, b \in L^{r}((a, b) \times \Omega, \tilde{\mathfrak{m}})$ for every $0<a<b<1$ and some $r \in[1, \infty)$ then

$$
\mathrm{u}(t, \cdot) \in L^{r}(\Omega), \quad \lim _{\tau \downarrow 0}\left\|\mathrm{u}_{\tau}(t, \cdot)-\mathrm{u}(t, \cdot)\right\|_{L^{r}(\Omega, \mathfrak{m})}=0 \quad \text { for every } t \in(0,1) .
$$

Proof. Let us first consider the equivalence of properties $(i),(i i)$, and $(i i i)$. It is easy to check that $(i i i) \Rightarrow$ $(i i) \Rightarrow(i)$. Let us show the implication $(i) \Rightarrow($ iii $)$.

We consider a pair $(\zeta, Z) \in \mathscr{Z}$ so that $Z \circ u$ satisfies the integrability condition

$$
\int_{(a, b) \times F}|Z(u(t, x))| \mathrm{d} \mathfrak{m}(t, x)<\infty \quad \text { for every } 0<a<b<1, \quad F \in \mathscr{B}, \mathfrak{m}(F)<\infty,
$$

which is always satisfied if $(\zeta, Z) \in \mathscr{Z}_{c}$. We select a Borel representative $u$ such that $Z \circ u \in L_{\text {loc }}^{1}(I)$ for every $x \in \Omega$, we call $B_{\zeta}(t, x):=\int_{1 / 2}^{t} \zeta(u(s, x)) \tilde{\beta}(s, x) \mathrm{d} s$, and $w_{\zeta}(t, x):=Z \circ u-B_{\zeta}$. We fix $\zeta$ and we initially omit the dependence on $\zeta$ by writing $w:=w_{\zeta}$. It is easy to check that for every nonnegative $\eta \in C_{c}^{1}(I)$ and $\varphi \in B_{f}(\Omega)$

$$
\int_{\Omega_{I}} \eta^{\prime}(t) \varphi(x) w(t, x) \mathrm{d} \tilde{\mathfrak{m}}(t, x) \leq 0 .
$$

Since $w$ is Borel and $\Omega_{1}(w)=\Omega$, Fubini's theorem shows that for every nonnegative $\eta \in C_{c}^{1}(I)$ and $\varphi \in B_{f}(\Omega)$

$$
\begin{aligned}
& -\int_{\Omega_{I}} \eta^{\prime}(t) \varphi(x) w_{\tau}(t, x) \mathrm{d} \tilde{\mathfrak{m}}(t, x)=-\int_{\Omega}\left(\int_{0}^{1} \eta^{\prime}(t) w_{\tau}(t, x) \mathrm{d} t\right) \varphi(x) \mathrm{d} \mathfrak{m}(x) \\
& =\int_{\Omega}\left(\int_{0}^{1} \eta(t) \partial_{t} w_{\tau}(t, x) \mathrm{d} t\right) \varphi(x) \mathrm{d} \mathfrak{m}(x)=\int_{\Omega_{I}} \eta(t) \varphi(x) \partial_{t} w_{\tau}(t, x) \mathrm{d} \tilde{\mathfrak{m}}(t, x) \geq 0
\end{aligned}
$$

We deduce that $\partial_{t} w_{\tau} \geq 0 \tilde{\mathfrak{m}}$-a.e. in $\Omega_{I}$ and therefore there exists a m-negligible set $N(\tau) \subset \Omega$ such that $\partial_{t} w_{\tau}(\cdot, x) \geq 0 \mathscr{L}^{1}$-a.e. in $\mathbb{R}$ for every $x \in \Omega \backslash N(\tau)$.

Since $\partial_{t} w_{\tau}(\cdot, x)$ is a continuous function, we conclude that $\partial_{t} w_{\tau}(\cdot, x) \geq 0$ in $I$ for every $x \in \Omega \backslash N(\tau)$. Setting $z(r):=r h(r)$, a simple calculation shows that for $0<\tau^{\prime}<\tau^{\prime \prime}$

$$
h_{\tau^{\prime \prime}}(r)-h_{\tau^{\prime}}(r)=-\int_{\tau^{\prime}}^{\tau^{\prime \prime}} \frac{1}{\tau^{2}} z(r / \tau) \mathrm{d} \tau
$$

so that

$$
w_{\tau^{\prime \prime}}(t, x)-w_{\tau^{\prime}}(t, x)=-\int_{\tau^{\prime}}^{\tau^{\prime \prime}} \frac{1}{\tau^{2}} \int_{\mathbb{R}} z((t-s) / \tau) w(s, x) \mathrm{d} s \mathrm{~d} \tau
$$

and a further integration w.r.t. $\mathfrak{m}$ with weight $\varphi$ yields

$$
\int_{\Omega}\left(w_{\tau^{\prime \prime}}(t, x)-w_{\tau^{\prime}}(t, x)\right) \varphi(x) \mathrm{d} \mathfrak{m}(x)=-\int_{\tau^{\prime}}^{\tau^{\prime \prime}} \frac{1}{\tau^{2}}\left(\int_{\Omega_{I}} z((t-s) / \tau) \varphi(x) w(s, x) \mathrm{d} \tilde{\mathfrak{m}}\right) \mathrm{d} \tau \geq 0
$$

since $z \leq 0$. It follows that there exists a m-negligible set $N\left(\tau^{\prime}, \tau^{\prime \prime}\right) \supset N\left(\tau^{\prime}\right) \cup N\left(\tau^{\prime \prime}\right)$ such that $w_{\tau^{\prime}}(t, x) \leq$ $w_{\tau^{\prime \prime}}(t, x)$ for every rational $t \in I \cap \mathbb{Q}$ and $x \in \Omega \backslash N\left(\tau^{\prime}, \tau^{\prime \prime}\right)$. Since $w_{\tau^{\prime}}$ and $w_{\tau^{\prime \prime}}$ are also continuous w.r.t. $t$ if $x \in \Omega \backslash N\left(\tau^{\prime}, \tau^{\prime \prime}\right)$ we conclude that $w_{\tau^{\prime}}(t, x) \leq w_{\tau^{\prime \prime}}(t, x)$ for every $t \in(0,1)$ and $x \in \Omega \backslash N\left(\tau^{\prime}, \tau^{\prime \prime}\right)$. 
By an induction argument, we can therefore select a decreasing sequence $\tau_{n} \downarrow 0$ and $\mathfrak{m}$-negligible set $N$ such that $t \mapsto w_{\tau_{n}}(t, x)$ is continuous and increasing w.r.t. $t$ in $I$ and $n \mapsto w_{\tau_{n}}(t, x)$ is decreasing for every $x \in \Omega \backslash N$. We can thus define

$$
\mathrm{w}(t, x):=\lim _{n \rightarrow \infty} w_{\tau_{n}}(t, x) \quad \text { if } x \in \Omega \backslash N ; \quad \mathrm{w}(t, x)=0 \text { otherwise. }
$$

Clearly $\mathrm{w}$ is increasing for every $x$; moreover, Lebesgue theorem shows that $\mathrm{w}(t, x)=w(t, x) \mathscr{L}^{1}$-a.e. in $(0,1)$ for every $x \in \Omega \backslash N$, so that $\mathrm{w}$ is a Borel representative of $w$. Finally, $\mathrm{w} * h_{\tau}(\cdot, x)=w * h_{\tau}(\cdot, x)=w_{\tau}(\cdot, x)$ in $I$ for every $x \in \Omega \backslash N$ so that $\mathrm{w}$ is right continuous.

Let us now turn back to write $w=w_{\zeta}, \mathrm{w}=\mathrm{w}_{\zeta}$ and try to remove the dependence on $\zeta$. We consider an increasing sequence $\zeta_{n}(r)=\chi\left(\left|2^{-n} r\right|\right)$ where $\chi:[0, \infty) \rightarrow[0,1]$ is a smooth decreasing function such that $\chi_{\left.\right|_{[0,1]}} \equiv 1$ and $\chi_{\left.\right|_{[2, \infty)}} \equiv 0$. Clearly $Z_{n}(r) \equiv r$ if $r \in\left[-2^{n}, 2^{n}\right]$ and $Z_{n} \circ Z_{n+1}=Z_{n}$. We set $w_{n}:=w_{\zeta_{n}}, B_{n}:=$ $B_{\zeta_{n}}$, and we call $N(n)$ the exceptional set $N\left(\zeta_{n}\right)$ we have previously found with $\bar{N}:=\cup_{n \in \mathbb{N}} N(n)$. It is easy to check that $B_{n}(\cdot, x) \rightarrow B(\cdot, x)$ locally uniformly in $I$ for every $x \in \Omega$ so that $S_{n}(x, a, b):=\sup _{x \in(a, b)} \mid B_{n}(\cdot, x)-$ $B(\cdot, x) \mid \rightarrow 0$ as $n \rightarrow \infty$. Setting $\mathrm{u}_{n}:=\mathrm{w}_{n}+B_{n}$ we clearly have $\mathrm{u}_{n}(\cdot, x)=Z_{n}(u(\cdot, x))=Z_{n}\left(Z_{n+1}(u(\cdot, x))\right)$ $\mathscr{L}^{1}$-a.e. in $I$ for every $x \in \Omega \backslash \bar{N}$, so that the right continuity of $\mathrm{u}_{n}$ yields $\mathrm{u}_{n}(\cdot, x)=Z_{n}\left(\mathrm{u}_{n+1}(\cdot, x)\right)$ everywhere in $I$ for every $x \in \Omega \backslash \bar{N}$. For every $0<a<b<1$ and $x \in \Omega \backslash \bar{N}$ there exist $a^{\prime} \in(0, a), b^{\prime} \in(b, 1)$ and $\bar{n}$ sufficiently big so that $\mathrm{u}_{n}\left(a^{\prime}, x\right)=Z_{n}\left(u\left(a^{\prime}, x\right)\right), \mathrm{u}_{n}\left(b^{\prime}, x\right)=Z_{n}(u(b, x)), S_{n}(x, a, b) \leq 1$ for every $n \geq \bar{n}$. We obtain the estimate

$$
\left(u\left(a^{\prime}, x\right) \wedge 0\right)-B\left(a^{\prime}, x\right)-1 \leq \mathrm{u}_{n}(t, x)-B(t, x) \leq\left(u\left(b^{\prime}, x\right) \vee 0\right)-B\left(b^{\prime}, x\right)+1 \quad \text { if } t \in(a, b), n \geq \bar{n} .
$$

We deduce that the sequence $n \mapsto \mathrm{u}_{n}(\cdot, x)$ is definitely constant in every interval $(a, b)$ and therefore the limits $\mathrm{u}(t, x)=\lim _{n \rightarrow \infty} \mathrm{u}_{n}(t, x), \mathrm{w}(t, x)=\lim _{n \rightarrow \infty} \mathrm{w}_{n}(t, x)$ exist for every $t \in I$ and define right continuous functions for every $x \in \Omega \backslash \bar{N}$ which satisfy $\mathrm{u}(t, x)=\mathrm{w}(t, x)+B(t, x)$. Since $\mathrm{w}$ is increasing w.r.t. $t$ we conclude.

(I.1) and (I.3) follow by the above construction. If now $\tilde{u}$ satisfies (4.13), since $\mathbf{u}=\tilde{u} \tilde{\mathfrak{m}}$-a.e. in $I \times \Omega$, we may find a subset $D_{1} \subset D$ of full measure such that $\tilde{u}(t, \cdot)=\mathrm{u}(t, \cdot) \mathfrak{m}$-a.e. in $\Omega$ for every $t \in D_{1}$. By Lemma 4.2 we conclude that $\tilde{u}^{+}(t, \cdot)=\mathrm{u}(t, \cdot)$ for every $t \in I$, so that (4.14) holds.

Concerning (I.4) it is clear by a limit procedure as we did in the previous claim that if (4.12) holds for every $(\zeta, Z) \in \mathscr{Z}$ and $u \in L^{1}((a, b) \times \Omega)$ for every $0<a<b<1$, then (4.12) holds also choosing $\zeta \equiv 1$ and the identity map as $Z$. To prove the converse implication, we simply observe that in the above proof $(i) \Rightarrow(i i i)$ we are allowed to choose at the beginning $\zeta=1$ so that the argument directly shows $(i i i)$ and therefore $(i)$ for arbitrary $(\zeta, Z) \in \mathscr{Z}_{c}$.

If eventually $u \in L^{p}((a, b) \times \Omega, \tilde{\mathfrak{m}})$ for every $0<a<b<1$ we deduce by Fubini's theorem that the set $t \in I: \mathbf{u}(t, \cdot) \in L^{p}(\Omega, \mathfrak{m})$ is of full measure in $I$, in particular there are sequences $a_{n} \downarrow 0$ and $b_{n} \uparrow 1$ such that $\mathrm{u}\left(a_{n}, \cdot\right), \mathbf{u}\left(b_{n}, \cdot\right) \in L^{p}(\Omega ; \mathfrak{m})$. It is also easy to check that $B(t, \cdot) \in L^{p}\left(\mathbb{R}^{d}\right)$ for every $t \in I$, so that we can then use the obvious bounds

$$
\left(\mathrm{u}\left(a_{n}, \cdot\right)-B\left(a_{n}, \cdot\right)\right) \wedge 0 \leq \mathrm{u}(t, \cdot)-B(t, \cdot) \leq\left(\mathrm{u}\left(b_{n}, \cdot\right)-B\left(b_{n}, \cdot\right)\right) \vee 0 \quad \text { if } a_{n} \leq t \leq b_{n}
$$

to show that the negative and the positive part of $\mathrm{u}(t, \cdot)$ are uniformly bounded by fixed functions in $L^{p}(\Omega, \mathfrak{m})$ in every compact interval of $(0,1)$. (4.15) then follows by Lebesgue's Dominated Convergence Theorem.

Remark 4.4 It is clear that when $\Omega=\mathbb{R}^{d}$ and $\mathfrak{m}=\mathscr{L}^{d}$ 4.12 can be equivalently formulated as

$$
-\partial_{t} Z(u) \leq \zeta(u) \beta \quad \text { in } \mathscr{D}^{\prime}(Q) \quad \text { for every }(\zeta, Z) \in \mathscr{Z}_{c} \text {. }
$$

If moreover $u \in L_{\mathrm{loc}}^{1}(Q)$ then claim (I.4) shows that we can equivalently write

$$
-\partial_{t} u \leq \beta \quad \text { in } \mathscr{D}^{\prime}(Q)
$$

Notice eventually that if $\left(\zeta_{i}, Z_{i}\right) \in \mathscr{Z}_{c}$ with $\zeta_{1} \leq \zeta_{2} \leq 1$ then (4.20) yields

$$
0 \leq \partial_{t} Z_{1}(u)+\zeta_{1}(u) \beta \leq \partial_{t} Z_{2}(u)+\zeta_{2}^{\prime}(u) \beta \leq \partial_{t} u+\beta \quad \text { in } \mathscr{D}^{\prime}(Q) .
$$

Indeed, (4.21) follows immediately from (4.19) with the choices $\zeta(r):=\zeta_{1}(r), \zeta(r):=\zeta_{2}(r)-\zeta_{1}(r)$ and $\zeta(r):=r-\zeta_{2}(r)$.

We now study the traces of $u$ at $t=0$ and $t=1$, by assuming that $\beta$ satisfies

$$
\int_{I \times F}|\beta(t, x)| \mathrm{d} \tilde{\mathfrak{m}}(t, x)<\infty \quad \text { for every } F \in \mathscr{B}, \mathfrak{m}(F)<\infty,
$$


so that we may select a Borel representative $\tilde{\beta}$ of $\beta$ such that

$$
\tilde{\beta}(\cdot, x) \in L^{1}(I), \quad B(t, x):=\int_{1 / 2}^{t} \tilde{\beta}(s, x) \mathrm{d} s, B(\cdot, x) \in \mathrm{AC}([0,1]) \quad \text { for every } x \in \Omega .
$$

Lemma 4.5 (Traces at $t=0$ and $t=1)$ Let $u, \beta \in L^{0}(I \times \Omega, \tilde{\mathfrak{m}})$ be satisfying (4.22) and one of the equivalent conditions of Lemma 4.3 and let $w:=u-B$. Let $w_{0}^{+}, w_{1}^{-}$be defined as in Lemma 4.2 starting from a Borel representative $\tilde{w}(t, \cdot)=\tilde{u}(t, \cdot)-B(t, \cdot)$ satisfying (4.13) and let

$$
\begin{aligned}
u_{0}^{+}:=w_{0}^{+}+B(0, \cdot) \in L^{0}(\Omega ; \mathbb{R} \cup\{-\infty\}) \quad u_{1}^{-}:=w_{1}^{-}+B(1, \cdot) \in L^{0}(\Omega ; \mathbb{R} \cup\{+\infty\}), \\
\mathbf{u}^{+}(0, x):=\lim _{t \downarrow 0} \mathrm{u}(t, x) \in[-\infty,+\infty), \quad \mathbf{u}^{-}(1, x):=\lim _{t \uparrow 1} \mathrm{u}(t, x) \in(-\infty,+\infty] \quad \text { for every } x \in \Omega .
\end{aligned}
$$

(i) We have

$$
u_{0}^{+}=\mathrm{u}^{+}(0, \cdot), \quad u_{1}^{-}=\mathrm{u}^{-}(1, \cdot) \quad \mathfrak{m} \text {-a.e. in } \Omega,
$$

(ii) If there exists $u_{0}, u_{1} \in L^{0}(\Omega, \mathfrak{m} ; \overline{\mathbb{R}})$ such that for every nonnegative $\eta \in C_{c}^{1}(\mathbb{R})$ and $\varphi \in B_{f}(\Omega)$ and $(\zeta, Z) \in \mathscr{Z}_{c}$

$$
\begin{aligned}
\eta(0) \int_{\Omega} Z\left(u_{0}\right) \varphi \mathrm{d} \mathfrak{m} & -\eta(1) \int_{\Omega} Z\left(u_{1}\right) \varphi \mathrm{d} \mathfrak{m} \\
& \leq \int_{\Omega_{I}}\left(\eta(t) \varphi(x) \zeta(u(t, x)) \beta(t, x)-\eta^{\prime}(t) \varphi(x) Z(u(t, x))\right) \mathrm{d} \tilde{\mathfrak{m}}(t, x)
\end{aligned}
$$

then

$$
u_{0} \leq u_{0}^{+}=\mathrm{u}^{+}(0, \cdot), \quad u_{1} \geq u_{1}^{-}=\mathrm{u}^{-}(1, \cdot) \quad \mathfrak{m} \text {-a.e. in } \Omega .
$$

(iii) (4.27) always holds with $u_{0}, u_{1}$ replaced by $u_{0}^{+}, u_{1}^{-}$respectively.

(iv) If (4.27) holds with $u_{0} \wedge 0, u_{1} \vee 0 \in L^{1}(\Omega, \mathfrak{m})$ then $u \in L^{1}(I \times \Omega ; \tilde{\mathfrak{m}})$ and

$$
\eta(0) \int_{\Omega} u_{0} \varphi \mathrm{d} \mathfrak{m}-\eta(1) \int_{\Omega} u_{1} \varphi \mathrm{d} \mathfrak{m} \leq \int_{\Omega_{I}}\left(\eta(t) \varphi(x) \beta(t, x)-\eta^{\prime}(t) \varphi(x) u(t, x)\right) \mathrm{d} \tilde{\mathfrak{m}}(t, x) .
$$

Proof. (4.26) is an immediate consequence of (4.14) and of the definition of convergence in measure.

In order to check $(i i)$, we take a smooth function $\chi \in C_{c}^{\infty}(\mathbb{R})$ taking values in $[0,1]$, supported in $(-1,1)$, satisfying $\chi \equiv 1$ in $[-1 / 2,1 / 2]$, and decreasing in $(0,1)$. We set $\chi_{\tau}(t):=\chi(t / \tau)$ and observe that as $\tau \downarrow 0$

$$
\begin{aligned}
-\int_{0}^{1} \chi_{\tau}^{\prime}(t) Z(\mathrm{u}(t, x)) \mathrm{d} t & =-\int_{0}^{1} Z(\mathrm{u}(\tau s, x)) \chi^{\prime}(s) \mathrm{d} s \rightarrow Z\left(\mathrm{u}^{+}(0, x)\right) \int_{0}^{1} \chi^{\prime}(s) \mathrm{d} s=Z\left(\mathrm{u}^{+}(0, x)\right), \\
\int_{0}^{1} \chi_{\tau}(t) \zeta(\mathrm{u}(t, x)) \tilde{\beta}(t, x) \mathrm{d} t & \rightarrow 0
\end{aligned}
$$

a further integration w.r.t. $x$ after a multiplication by $\varphi$ and the Lebesgue's Dominated Convergence Theorem yield

$$
\int_{\Omega} Z\left(u_{0}\right) \varphi \mathrm{d} \mathfrak{m} \leq-\int_{\Omega}\left(\int_{0}^{1} \chi_{\tau}^{\prime}(t) Z(\mathrm{u}(t, x)) \mathrm{d} t-\int_{0}^{1} \chi_{\tau}(t) \zeta(\mathrm{u}(t, x)) \tilde{\beta}(t, x) \mathrm{d} t\right) \varphi \mathrm{d} \mathfrak{m}(x) \rightarrow \int_{\Omega} Z\left(\mathbf{u}^{+}(0, \cdot)\right) \varphi \mathrm{d} \mathfrak{m}
$$

Since $\varphi$ is arbitrary, we get (4.28).

In order to prove $(i i i)$ it is not restrictive to consider the case when $\eta(0)=1$ and $\operatorname{supp}(\eta) \subset(-\infty, 1)$. If $\chi$ is as in the previous step, we can define $\eta_{\tau}^{0}:=\eta \chi_{\tau}, \eta_{\tau}^{1}:=\eta\left(1-\chi_{\tau}\right)$, observing that $\eta_{\tau}^{1} \in C_{c}^{1}(I)$ and therefore

$$
\begin{aligned}
& \int_{0}^{1}\left(\eta(t) \zeta(\mathrm{u}(t, x)) \tilde{\beta}(t, x)-\eta^{\prime}(t) Z(\mathrm{u}(t, x))\right) \mathrm{d} t \geq \int_{0}^{1}\left(\eta_{\tau}^{0}(t) \zeta(\mathrm{u}(t, x)) \tilde{\beta}(t, x)-\left(\eta_{\tau}^{0}\right)^{\prime}(t) Z(\mathrm{u}(t, x))\right) \mathrm{d} t \\
& =\int_{0}^{\tau} \chi(t / \tau)\left(\eta(t) \zeta(\mathrm{u}(t, x)) \tilde{\beta}(u(t, x))-\eta^{\prime}(t) Z(\mathrm{u}(t, x))\right) \mathrm{d} t-\int_{0}^{1} \eta(\tau s) Z(\mathrm{u}(\tau s, x)) \chi^{\prime}(s) \mathrm{d} s \rightarrow \eta(0) Z\left(\mathrm{u}^{+}(0, x)\right)
\end{aligned}
$$

An integration w.r.t. $\varphi \mathfrak{m}$ and another application of Lebesgue's Dominated Convergence Theorem yield

$$
\int_{I \times \Omega}\left(\eta(t) \zeta(u(t, x)) \beta(t, x)-\eta^{\prime}(t) Z(\mathrm{u}(t, x))\right) \varphi(x) \mathrm{d} \tilde{\mathfrak{m}}(t, x) \geq \eta(0) \int_{\Omega} Z\left(\mathbf{u}^{+}(0, x)\right) \varphi(x) \mathrm{d} \mathfrak{m}(x) .
$$

Finally, for what concerns (iv), arguing as in the proof of the last statement of Lemma 4.3, if $u_{0} \wedge 0, u_{1} \vee 0$ are integrable w.r.t. $\mathfrak{m}$ we deduce the integrability of $u$ in $I \times \Omega$. We can then write (4.27) for a sequence $Z_{n}(t)=\int_{0}^{t} \zeta_{n}(r) \mathrm{d} r$ with $\zeta_{n} \uparrow 1$ as $n \rightarrow \infty$; passing to the limit as $n \rightarrow \infty$ we get $Z_{n}(u) \rightarrow u$ pointwise $\mathfrak{m}$-almost everywhere and (4.29). 


\subsection{Convolution by anisotropic kernels}

When we deal with functions defined in the cylinder $Q$ we will also use space convolutions, induced by

$$
k \in C_{c}^{\infty}\left(\mathbb{R}^{d}\right), \quad k \geq 0, \quad \operatorname{supp} k \subset B_{1}(0), \quad \int_{\mathbb{R}^{d}} k \mathrm{~d} x=1,
$$

setting for $\varepsilon, \tau>0($ recall 4.10$)$ )

$$
k_{\varepsilon}(x):=\varepsilon^{-d} k(x / \varepsilon), \quad \eta_{\tau, \varepsilon}(t, x):=h_{\tau}(t) k_{\varepsilon}(x)
$$

If $u \in L_{\text {loc }}^{1}(Q)$ then the space-time convolution in $\mathbb{R}^{d+1}$

$$
u_{\tau, \varepsilon}:=u * \eta_{\tau, \varepsilon} \text { is well defined and smooth in }(0,1-\tau) \times \mathbb{R}^{d} .
$$

We already defined the partial convolution $u_{\tau}:=u *_{t} \eta_{\tau}$ by using an everywhere defined Borel representative $\tilde{u}$ of $u$; the next Lemma shows that we can select an even better representative of $u_{\tau}$ which behaves nicely w.r.t. $u_{\tau, \varepsilon}$.

Lemma 4.6 Let $u \in L_{\mathrm{loc}}^{1}(Q)$. There exists a $\mathscr{L}^{d}$-negligible set $N \subset \mathbb{R}^{d}$ and, for every $\tau>0$, a measurable map $\mathrm{u}_{\tau}:(0,1-\tau) \times \mathbb{R}^{d} \rightarrow \mathbb{R}$ which coincides with $u_{\tau} \lambda$-a.e. in $Q$ and satisfies the following properties:

(1) The maps $t \mapsto \mathrm{u}_{\tau}(t, x)$ belong to $C^{\infty}(0,1-\tau)$ for every $x \in \mathbb{R}^{d} \backslash N$ with

$$
\int_{F}\left\|\mathrm{u}_{\tau}(\cdot, x)\right\|_{L^{\infty}(a, b)} \mathrm{d} x<\infty \quad \text { for every bounded } F \subset \mathbb{R}^{d} \text { and for every } 0<a<b<1-\tau,
$$

and the function

$$
t \mapsto \mathbf{u}_{\tau}(t, \cdot) \text { defined in }(0,1-\tau) \text { with values in } L_{\mathrm{loc}}^{1}\left(\mathbb{R}^{d}\right) \text { is continuous. }
$$

(2) For every bounded measurable $F \in \mathbb{R}^{d}$ and every $0<a<b<1-\tau$

$$
\lim _{\varepsilon \downarrow 0} \int_{F}\left(\sup _{s \in[a, b]}\left|u_{\tau, \varepsilon}(s, x)-\mathbf{u}_{\tau}(s, x)\right|\right) \mathrm{d} x=0 .
$$

In particular

$$
\lim _{\varepsilon \downarrow 0} \sup _{s \in[a, b]} \int_{F}\left(\left|u_{\tau, \varepsilon}(s, x)-\mathbf{u}_{\tau}(s, x)\right|\right) \mathrm{d} x=0, \quad \lim _{\varepsilon \downarrow 0} \int_{a}^{b} \int_{F}\left|u_{\tau, \varepsilon}(s, x)-\mathbf{u}_{\tau}(s, x)\right| \mathrm{d} s \mathrm{~d} x=0,
$$

and

$$
u_{\tau, \varepsilon}(t, \cdot) \rightarrow \mathrm{u}_{\tau}(t, \cdot) \text { in } L_{\mathrm{loc}}^{1}\left(\mathbb{R}^{d}\right) \text { as } \varepsilon \downarrow 0 \text { for every } t \in(0,1-\tau) \text {. }
$$

(3) $\lim _{\varepsilon \downarrow 0} u_{\tau, \varepsilon}(\cdot, x)=\mathbf{u}_{\tau}(\cdot, x)$ locally uniformly in $C(0,1-\tau)$ for every $x \in \mathbb{R}^{d} \backslash N$.

(4) $\lim _{\tau \downarrow 0} \mathrm{u}_{\tau}=u$ in $L_{\mathrm{loc}}^{1}(Q)$.

If moreover $u$ satisfies one of the equivalent properties of Lemma 4.3 then we may assume that $\mathbf{u}_{\tau}(\cdot, x)$ coincides with the precise representative of Lemma 4.3 for every $x \in \mathbb{R}^{d} \backslash N$.

Proof. Let us consider an increasing sequence of intervals $\left[a_{n}, b_{n}\right] \uparrow(0,1)$ as $n \rightarrow \infty$ and an increasing sequence of bounded open balls $B_{k}$ centered at 0 and of radious $k$, so that $B_{k} \uparrow \mathbb{R}^{d}$ as $k \rightarrow \infty$. By selecting a Borel representative $\tilde{w}: Q \rightarrow \mathbb{R}$ of $u$ and applying Fubini's theorem to the restrictions $u_{n, k}$ of $\tilde{u}$ to $\left(a_{n}, b_{n}\right) \times B_{k+1}$, we obtain a countable family of strongly measurable maps $u_{n, k}: B_{k+1} \rightarrow L^{1}\left(a_{n}, b_{n}\right)$ which belong to the Lebesgue-Bochner space $L^{1}\left(B_{k+1} ; L^{1}\left(a_{n}, b_{n}\right)\right)$. We can thus consider the set of Lebesgue points $L_{n, k}$ of $u_{n, k}$ in $B_{k}$ : we know that $\mathscr{L}^{d}\left(B_{k} \backslash L_{n, k}\right)=0$ and each $\bar{x} \in L_{n, k}$ satisfies

$$
u_{n}(\cdot, \bar{x}) \in L^{1}\left(a_{n}, b_{n}\right), \quad \lim _{r \downarrow 0}\left\|\int_{B_{r}(\bar{x})} u_{n}(\cdot, x) \mathrm{d} x-u_{n}(\cdot, \bar{x})\right\|_{L^{1}\left(a_{n}, b_{n}\right)}=0 .
$$

We thus set $N:=\bigcup_{k, n \in \mathbb{N}}\left(B_{k} \backslash L_{n, k}\right)$ and

$$
\mathrm{u}_{\tau}(t, x):=\int_{\mathbb{R}} \tilde{u}(s, x) h_{\tau}(t-s) \mathrm{d} s \quad t \in(0,1-\tau), \quad x \in \mathbb{R}^{d} \backslash N ; \quad \mathbf{u}_{\tau}(t, x)=0 \text { if } x \in N .
$$


Let us now fix $\tau>0$, an interval $(a, b)$ with $0<a<b<1-\tau$, a bounded measurable set $F \subset \mathbb{R}^{d}$, and integers $n, k$ sufficiently big so that $(a, b+\tau) \subset\left(a_{n}, b_{n}\right)$ and $F \subset B_{k}$ and therefore the restriction of $\tilde{u}$ to $(a, b) \times F$ coincides with the restriction of $\bar{u}:=u_{n, k} ;$ in particular

$$
\mathrm{u}_{\tau}(t, x)=\bar{u}_{\tau}(t, x)=\int_{a_{n}}^{b_{n}} \bar{u}(s, x) h_{\tau}(t-s) \mathrm{d} s \quad \text { if } t \in(a, b), \quad x \in F \backslash N .
$$

By Fubini's theorem we know that $\int_{F}\|\bar{u}\|_{L^{1}(a, b)} \mathrm{d} x<\infty$, so that the maps $t \mapsto \mathrm{u}_{\tau}(t, x)$ belong to $C^{\infty}(a, b)$ for every $x \in F \backslash N$ and satisfies (4.33). In order to check (4.34) we can use the Lebesgue Dominated Convergence theorem and the estimate (4.33), since for every $x \in F \backslash N$ and $t \in(a, b)$ we have $\lim _{s \rightarrow t} \mathbf{u}_{\tau}(s, x)=\mathbf{u}_{\tau}(t, x)$ and if $s \in(a, b)\left|\mathrm{u}_{\tau}(s, x)\right| \leq\|\mathrm{u}(\cdot, x)\|_{L^{\infty}(a, b)}$. Being $F$ and $(a, b)$ arbitrary we conclude the proof of the first claim.

Let us now consider the claim (2). Since the linear map $\zeta \mapsto \zeta * h_{\tau}$ is well defined (and continuous) from $L^{1}\left(a_{n}, b_{n}\right)$ to $C([a, b])$, the map $\bar{u}_{\tau}^{x}:=\bar{u}(\cdot, x) * h_{\tau}$ is strongly measurable from $B_{k+1}$ to $C([a, b])$ and the set of its Lebesgue points surely contains $F \backslash N$. Performing a convolution w.r.t. $x \in \mathbb{R}^{d}$ with the kernel $k_{\varepsilon}, \varepsilon<1$, and values in the Banach space $C([a, b])$ we thus obtain a continuous map $x \mapsto \bar{u}_{\tau, \varepsilon}^{x}$ from $B_{k}$ to $C([a, b])$; $\bar{u}_{\tau, \varepsilon}^{x}$ is defined by the Bochner integral $\bar{u}_{\tau, \varepsilon}^{x}(t):=\int_{\mathbb{R}^{d}} \bar{u}_{\tau}^{y}(t) k_{\varepsilon}(x-y) \mathrm{d} y$ and it is not difficult to check that $\bar{u}_{\tau, \varepsilon}^{x, \varepsilon}(t)=u_{\tau, \varepsilon}(t, x)$ for every $x \in B_{k}$ and $t \in[a, b]$.

(4.35) then follows by general results on convolutions for Banach-valued functions. (4.36) are obvious consequences of (4.35) and (4.37) follows by the first limit of (4.36).

In order to check the third claim, we apply Lebesgue theorem for the Bochner integral in the Banach space $C([a, b])$ : at every Lebesgue point $x \in B_{k} \backslash N$

$$
\lim _{\varepsilon \downarrow 0} \bar{u}_{\tau, \varepsilon}^{x}=\bar{u}_{\tau}^{x} \quad \text { uniformly in } C([a, b]) .
$$

Claim (4) can be proved by adapting the well known result for convolution with compactly supported kernels.

Finally, if $\mathrm{u}_{\tau}^{\prime}$ is the precise representative of Lemma 4.3, up to modifying $N$ by a further $\mathscr{L}^{d}$-negligible set, we know that $\mathrm{u}_{\tau}(\cdot, x)=\mathrm{u}_{\tau}^{\prime}(\cdot, x) \mathscr{L}^{1}$-a.e. in $(0,1)$ for every $x \in N$. Since both the functions are continuous w.r.t. $t \in(0,1)$ they should coincide.

\subsection{The space $L^{q}+L_{1 / \kappa}^{\infty}(\Omega)$}

Let us consider an open set $\Omega \subset \mathbb{R}^{h}$ and a measurable weight $\omega: \Omega \rightarrow[1, \infty)$. As we said in Section 2.1 our main examples will be $\Omega=\mathbb{R}^{d}$ or $\Omega=Q$ with the weight $\kappa(x):=1+|x|^{2}$ of (2.4).

The space $L^{p} \cap L_{\omega}^{1}(\Omega)$ can be endowed with the Banach norm

$$
\|g\|_{L^{p} \cap L_{\omega}^{1}(\Omega)}:=\max \left(\|g\|_{L_{\omega}^{1}(\Omega)},\|g\|_{L^{p}(\Omega)}\right) .
$$

Its dual admits a sum representation.

Definition 4.7 Let $\omega: \Omega \rightarrow[1, \infty)$ a measurable weight. We call $\mathcal{X}_{\omega}^{q}(\Omega):=L^{q}(\Omega)+L_{1 / \omega}^{\infty}(\Omega)$ the space of functions $v \in L^{0}(\Omega)$ admitting a decomposition

$$
v=w+z, \quad w \in L_{1 / \omega}^{\infty}(\Omega), z \in L^{q}(\Omega),
$$

with norm $\|v\|_{\mathcal{X}_{\omega}^{q}(\Omega)}:=\inf \left\{\|w\|_{L_{1 / \omega}^{\infty}(\Omega)}+\|z\|_{L^{q}(\Omega)}: v=w+z\right\}$.

We simply denote by $\mathcal{X}^{q}(\Omega)$ the space defined when $\omega$ is the standard weight $\kappa=1+|x|^{2}$.

We can equivalently characterize the norm of $\mathcal{X}_{\omega}^{q}(\Omega)$ as the inf-convolution

$$
\|v\|_{\mathcal{X}_{\omega}^{q}(\Omega)}=\inf _{z \in L^{q}(\Omega)}\|v-z\|_{L_{1 / \omega}^{\infty}(\Omega)}+\|z\|_{L^{q}(\Omega)}
$$

(4.42) is also equivalent to

$$
v=\omega \tilde{w}+z, \quad \tilde{w} \in L^{\infty}(\Omega), z \in L^{q}(\Omega) .
$$

We collect in the next Lemma a list of useful properties of $\mathcal{X}_{\omega}^{q}(\Omega)$.

Lemma $4.8 \mathcal{X}_{\omega}^{q}(\Omega)$ is a Banach space which can be (isometrically) identified with the dual of $L^{p} \cap L_{\omega}^{1}(\Omega)$; in particular it holds

$$
\|v\|_{\mathcal{X}_{\omega}^{q}(\Omega)}=\sup \left\{\int_{\Omega} v g \mathrm{~d} x: g \in L^{p} \cap L_{\omega}^{1}(\Omega),\|g\|_{L^{p} \cap L_{\omega}^{1}(\Omega)} \leq 1\right\} .
$$

Moreover 
(a) The infimum in the defnition of the norm of $\mathcal{X}_{\omega}^{q}(\Omega)$ given by Definition 4.7 is attained and the minimizer is unique.

(b) If $v \geq 0$ we can restrict the infimum to nonegative pairs $w, z$.

(c) Similarly, it is not restrictive to assume that $w$ and $z$ share the same sign of $v$, i.e. $w, z \geq 0$ in $\{x \in \Omega: v \geq 0\}$ and $w, z \leq 0$ in $\{x \in \Omega: v \leq 0\}$.

(d) For every $v \in \mathcal{X}_{\omega}^{q}(\Omega)$ the function $|v|$ belongs to $\mathcal{X}_{\omega}^{q}(\Omega)$ and $\|v\|_{\mathcal{X}_{\omega}^{q}}=\||v|\|_{\mathcal{X}_{\omega}^{q}}$.

(e) if $0 \leq v_{1} \leq v_{2}$ pointwise a.e. in $\Omega$ and $v_{2} \in \mathcal{X}_{\omega}^{q}(\Omega)$ then $v_{1} \in \mathcal{X}_{\omega}^{q}(\Omega)$ and $\left\|v_{1}\right\|_{\mathcal{X}_{\omega}^{q}} \leq\left\|v_{2}\right\|_{\mathcal{X}_{\omega}^{q}}$. In particular $\|v \wedge 0\|_{\mathcal{X}_{\omega}^{q}},\|v \vee 0\|_{\mathcal{X}_{\omega}^{q}} \leq\|v\|_{\mathcal{X}_{\omega}^{q}} \leq\|v \vee 0\|_{\mathcal{X}_{\omega}^{q}}+\|v \wedge 0\|_{\mathcal{X}_{\omega}^{q}}$.

(f) If $v \geq 0$ and there exists a constant $C \geq 0$ such that

$$
\int_{\Omega} v f \mathrm{~d} x \leq C \max \left(\int_{\Omega} \omega f \mathrm{~d} x,\|f\|_{L^{p}(\Omega)}\right),
$$

for every nonnegative $f \in L^{p} \cap L_{\omega}^{1}(\Omega)$ with bounded support, then $v \in \mathcal{X}_{\omega}^{q}(\Omega)$ and $\|v\|_{\mathcal{X}_{\omega}^{q}} \leq C$.

Proof. The duality with $L^{p} \cap L_{\omega}^{1}(\Omega)$ and (4.45) follows by a general result on the dual of the intersection of Banach spaces, see e.g. [5, Theorem 2.7.1]. It is also easy to check that the infimum is attained, since bounded sets in $L^{q}$ (resp. $L_{1 / \omega}^{\infty}$ ) are weakly (resp. weakly*) relatively compact and the norm is weakly (resp. weakly*) lower semicontinuous. Since the $L^{q}$ norm is strictly convex, the minimizer is unique.

In order to check (b) it is sufficient to notice that if $z, w$ satisfy (4.42) and $v \geq 0$, then $z_{1}:=0 \vee z \wedge v$ and $w_{1}:=v-z_{1}$ provide a pair of functions, still satisfying (4.42) with $0 \leq z_{1} \leq z \vee 0$ and $0 \leq w_{1} \leq w \vee 0$ so that $\left\|z_{1}\right\|_{L^{q}(\Omega)} \leq\|z\|_{L^{q}(\Omega)}$ and $\left\|w_{1}\right\|_{L_{1 / \omega}^{\infty}(\Omega)} \leq\|w\|_{L_{1 / \omega}^{\infty}(\Omega)}$. A similar argument, localized to the sets $\{x \in \Omega: v \geq 0\}$ and $\{x \in \Omega: v \leq 0\}$, yields (c).

Let us now consider statement (d); first of all, by the previous claim (c), if $v=w+z$ is the optimal decomposition of $v$ we have $|v|=|w|+|z|$ so that $\||v|\|_{\mathcal{X}_{\omega}^{q}} \leq\|v\|_{\mathcal{X}_{\omega}^{q}}$. On the other hand, if $|v|=w+z$ is the optimal decomposition of $|v|$, we have $v=w \operatorname{sign}(v)+z \operatorname{sign}(v)$ so that $\|v\|_{\mathcal{X}_{\omega}^{q}} \leq\||v|\|_{\mathcal{X}_{\omega}^{q}}$.

Claim (e) follows by the following remark: if $v_{2}=w_{2}+z_{2}$ is the optimal decomposition of $v_{2}$ (so that $z_{2}, w_{2}$ are nonnegative by (b)) we may set $z_{1}:=z_{2} \wedge v_{1}, w_{1}:=v_{1}-z_{1} \leq w_{2}$, obtaining an admissible decomposition for $v_{1}$ with $\left\|z_{1}\right\|_{L^{q}(\Omega)} \leq\left\|z_{2}\right\|_{L^{q}(\Omega)}$ and $\left\|w_{1}\right\|_{L_{1 / \omega}^{\infty}(\Omega)} \leq\left\|w_{2}\right\|_{L_{1 / \omega}^{\infty}(\Omega)}$ which shows $\left\|v_{1}\right\|_{\mathcal{X}_{\omega}^{q}} \leq\left\|v_{2}\right\|_{\mathcal{X}_{\omega}^{q}}$.

Finally, since functions with bounded support are dense in $L^{p} \cap L_{\omega}^{1}(\Omega)$ it is easy to see that (4.46) yields $v \in\left(L^{p} \cap L_{\omega}^{1}(\Omega)\right)^{\prime}=\mathcal{X}_{\omega}^{q}(\Omega)$; we can then apply (4.45).

\section{Weak subsolutions to Hamilton-Jacobi equations}

In this section we study some properties of weak subsolutions to Hamilton-Jacobi equations in $Q=(0,1) \times \mathbb{R}^{d}$.

\subsection{Weak subsolutions, precise representative and truncations}

We start with the following

Definition 5.1 Given an Hamiltonian $H$ satisfying assumptions 2.1 and a function $\alpha \in L_{\mathrm{loc}}^{1}(Q)$, a weak subsolution to the equation

$$
-\partial_{t} u+H(x, D u) \leq \alpha,
$$

is a function $u \in L_{\mathrm{loc}}^{1}(Q)$ with distributional gradient $D u \in L_{\mathrm{loc}}^{2}\left(Q ; \mathbb{R}^{d}\right)$ satisfying the inequality (5.1) in the sense of distributions, i.e.

$$
\int_{Q}\left(u \partial_{t} \xi+(H(x, D u)-\alpha) \xi\right) \mathrm{d} x \mathrm{~d} t \leq 0
$$

for every non-negative $\xi \in C_{c}^{\infty}(Q)$.

It is easy to check that any weak subsolution, according to the above definition, actually satisfies (5.2) for every $\xi \in C_{c}^{0}(Q)$ with $\partial_{t} \xi \in C_{c}^{0}(Q)$.

Remark 5.2 Thanks to the growth condition (H3) on the Hamiltonian (see Assumptions [2.1), many estimates can be derived by looking at the model case associated to the Hamiltonian $H(x, \boldsymbol{p}):=\frac{1}{2 c_{H}}|\boldsymbol{p}|^{2}$ and to the equation

$$
-\partial_{t} u+\frac{1}{2 c_{H}}|D u|^{2} \leq \beta .
$$

Notice indeed that if $u$ is a weak subsolution to (5.1) then $u$ is a subsolution to (5.3), with $\beta=\alpha+\gamma_{H}^{-}$. In particular, if $\alpha \in \mathcal{X}^{q}(Q)$ then also $\beta \in \mathcal{X}^{q}(Q)$. 
Weak subsolutions exhibit a nice behaviour with respect to truncations.

Lemma 5.3 (Truncation) Let $u_{i}, i=1,2$, be subsolutions of (5.1) with respect to $\alpha_{i} \in L_{\text {loc }}^{1}(Q)$, and let $\chi_{i}$ be the characteristic functions

$$
\chi_{1}:=\chi_{\left\{u_{1} \geq u_{2}\right\}}, \quad \chi_{2}:=\chi_{\left\{u_{2}>u_{1}\right\}}
$$

Then

$$
\begin{array}{llll}
u_{1} \vee u_{2} & \text { is a subsolution of (5.1) w.r.t. } & \chi_{1} \alpha_{1}+\chi_{2} \alpha_{2}, \\
u_{1} \wedge u_{2} & \text { is a subsolution of (5.1) w.r.t. } & \chi_{2} \alpha_{1}+\chi_{1} \alpha_{2} .
\end{array}
$$

Proof. We prove only (5.5), by adapting the classical Stampacchia's truncation argument to distributional inequalities. The proof of (5.6) is completely analogous.

Step 1: if $u_{i}, \beta_{i} \in L_{\text {loc }}^{1}(Q)$ satisfy $-\partial_{t} u_{i} \leq \beta_{i}$ in $\mathscr{D}^{\prime}(Q)$ then $-\partial_{t}\left(u_{1} \vee u_{2}\right) \leq \beta$ where $\beta:=\chi_{1} \beta_{1}+\chi_{2} \beta_{2}$ and $\chi_{i}$ are defined as in (5.4).

Recalling that for every $r_{1}, r_{2} \in \mathbb{R} r_{1} \vee r_{2}=r_{1}+\left(r_{2}-r_{1}\right) \vee 0$, we may consider the regularized truncations depending on $\varepsilon>0$

$$
S_{\varepsilon}\left(r_{1}, r_{2}\right):=r_{1}+T_{\varepsilon}\left(r_{2}-r_{1}\right), \quad T_{\varepsilon}(r):= \begin{cases}0 & \text { if } r \leq 0 \\ \sqrt{\varepsilon^{2}+r^{2}}-\varepsilon & \text { if } r>0\end{cases}
$$

whose derivatives $S_{\varepsilon, i}:=\frac{\partial}{\partial r_{i}} S_{\varepsilon}$ satisfy

$$
S_{\varepsilon, 1}\left(r_{1}, r_{2}\right)=\left\{\begin{array}{ll}
1 & \text { if } r_{1} \geq r_{2}, \\
1-\frac{r_{2}-r_{1}}{\sqrt{\varepsilon^{2}+\left(r_{2}-r_{1}\right)^{2}}} & \text { if } r_{2}>r_{1} ;
\end{array} \quad S_{\varepsilon, 2}\left(r_{1}, r_{2}\right)= \begin{cases}0 & \text { if } r_{1} \geq r_{2}, \\
\frac{r_{2}-r_{1}}{\sqrt{\varepsilon^{2}+\left(r_{2}-r_{1}\right)^{2}}} & \text { if } r_{2}>r_{1} .\end{cases}\right.
$$

Notice that $S_{\varepsilon}$ are of class $C^{1}$, Lipschitz, their derivatives satisfy $0 \leq S_{\varepsilon, i} \leq 1$, and

$$
S_{\varepsilon}\left(r_{1}, r_{2}\right) \uparrow r_{1} \vee r_{2}, \quad S_{\varepsilon, 1}\left(r_{1}, r_{2}\right) \downarrow \chi_{\left\{r_{1} \geq r_{2}\right\}}, \quad S_{\varepsilon, 1}\left(r_{1}, r_{2}\right) \uparrow \chi_{\left\{r_{1}<r_{2}\right\}} \quad \text { as } \varepsilon \downarrow 0 .
$$

Let $\eta^{\delta}, \delta>0$, be a usual family of nonnegative, smooth regularization kernels with compact support in $\mathbb{R}^{d+1}$.

Let us choose a nonnegative test function $\zeta \in C_{c}^{\infty}(Q)$ and an open subset $G$ with compact support in $Q$ such that $\operatorname{supp}(\zeta) \subset G$; choosing $\delta$ sufficiently small, $u_{i}^{\delta}:=u_{i} * \eta^{\delta}$ and $\beta_{i}^{\delta}:=\beta_{i} * \eta^{\delta}$ are well defined and smooth on $G$ and satisfy $-\partial_{t} u_{i}^{\delta} \leq \beta_{i}^{\delta}$ in the classical sense. Since $S_{\varepsilon, i} \geq 0$ we thus get

$$
-\partial_{t}\left(S_{\varepsilon}\left(u_{1}^{\delta}, u_{2}^{\delta}\right)\right)=-S_{\varepsilon, 1}\left(u_{1}^{\delta}, u_{2}^{\delta}\right) \partial_{t} u_{1}^{\delta}-S_{\varepsilon, 2}\left(u_{1}^{\delta}, u_{2}^{\delta}\right) \partial_{t} u_{2}^{\delta} \leq S_{\varepsilon, 1}\left(u_{1}^{\delta}, u_{2}^{\delta}\right) \beta_{1}^{\delta}+S_{\varepsilon, 2}\left(u_{1}^{\delta}, u_{2}^{\delta}\right) \beta_{2}^{\delta}
$$

pointwise in $G$, so that

$$
\int_{Q} S_{\varepsilon}\left(u_{1}^{\delta}, u_{2}^{\delta}\right) \partial_{t} \zeta \mathrm{d} t \mathrm{~d} x \leq \int_{Q}\left(S_{\varepsilon, 1}\left(u_{1}^{\delta}, u_{2}^{\delta}\right) \beta_{1}^{\delta}+S_{\varepsilon, 2}\left(u_{1}^{\delta}, u_{2}^{\delta}\right) \beta_{2}^{\delta}\right) \zeta \mathrm{d} t \mathrm{~d} x .
$$

We can first pass to the limit as $\delta \downarrow 0$, using the fact that $u_{i}^{\delta}$ (resp. $\beta_{i}^{\delta}$ ) converges to $u_{i}$ (resp. $\beta_{i}$ ) pointwise a.e. and strongly in $L^{1}(G)$, obtaining

$$
\int_{Q} S_{\varepsilon}\left(u_{1}, u_{2}\right) \partial_{t} \zeta \mathrm{d} t \mathrm{~d} x \leq \int_{Q}\left(S_{\varepsilon, 1}\left(u_{1}, u_{2}\right) \beta_{1}+S_{\varepsilon, 2}\left(u_{1}, u_{2}\right) \beta_{2}\right) \zeta \mathrm{d} t \mathrm{~d} x .
$$

We can eventually pass to the limit as $\varepsilon \downarrow 0$ using (15.7) and the uniform bounds $0 \leq S_{\varepsilon, i} \leq 1$.

Step 2: if $u_{i}, v_{i} \in L_{\mathrm{loc}}^{1}(Q)$ satisfy $\partial_{x_{k}} u_{i}=v_{i}$ in $\mathscr{D}^{\prime}(Q)$ then $\partial_{x_{k}}\left(u_{1} \vee u_{2}\right)=\chi_{1} v_{1}+\bar{\chi}_{2} v_{2}$ and $v_{1}=v_{2}$ a.e. on the set $\left\{u_{1}=u_{2}\right\}$.

This is a well-known property and, in any case, it follows from the previous claim (for $\partial_{x_{k}}$ instead of $\partial_{t}$ ), observing that a corresponding statement holds also for the inequalities $-\partial_{x_{k}} u_{i} \geq v_{i}$. The fact that $v_{1}=v_{2}$ a.e. on the set $\left\{u_{1}=u_{2}\right\}$ follows by interchanging the order of $u_{1}$ and $u_{2}$ in the formula.

Conclusion. We first apply claim 1 , choosing $\beta_{i}:=\alpha_{i}-H\left(x, D u_{i}\right)$ obtaining

$$
-\partial_{t}\left(u_{1} \vee u_{2}\right) \leq \chi_{1} \alpha_{1}+\chi_{2} \alpha_{2}-\left(\chi_{1} H\left(x, D u_{1}\right)+\chi_{2} H\left(x, D u_{2}\right)\right) ;
$$

we eventually observe that $\chi_{1}$ and $\chi_{2}$ are characteristic functions of a partition, so that $\chi_{1} H\left(x, D u_{1}\right)+$ $\chi_{2} H\left(x, D u_{2}\right)=H\left(x, D\left(u_{1} \vee u_{2}\right)\right)$ by the second claim. 
Corollary 5.4 Let $u$ be a subsolution of (5.1), $\alpha, u \in L_{\text {loc }}^{1}(Q)$. Then for every choice of $\sigma_{-}<\sigma_{+}$in $\mathbb{R}$ the function $u_{\sigma}:=\sigma_{-} \vee u \wedge \sigma_{+}$, is a weak subsolution to (5.1) with respect to the right hand side $\alpha_{\sigma}$ given by

$$
\alpha_{\sigma}:= \begin{cases}\alpha & \text { if } \sigma_{-} \leq u \leq \sigma_{+} \\ \gamma_{H}^{+} & \text {if } u<\sigma_{-} \text {or } u>\sigma_{+}\end{cases}
$$

Proof. It is sufficient to observe that every constant function is a subsolution to (5.1) with right hand side $\gamma_{H}^{+}$

The next lemma shows that partial time integration of functions $\beta \in \mathcal{X}^{q}(Q)$ naturally yields functions $B$ satisfying the assumptions used in Lemma 4.3 and 4.5

Lemma 5.5 If $\beta \in \mathcal{X}^{q}(Q)$ then $\beta$ satisfies (4.22) and (4.9) holds. Moreover, the map $t \mapsto B(t, \cdot)$ is $1 / p$ Hölder continuous with values in $\mathcal{X}^{q}\left(\mathbb{R}^{d}\right)$.

Proof. Let us decompose $\beta=\kappa \beta_{1}+\beta_{2}$ with $\beta_{1} \in L^{\infty}(Q)$ and $\beta_{2} \in L^{q}(Q)$. Since $\kappa$ does not depend on $t$, by Fubini's theorem we can find a Borel representative $\tilde{\beta}_{i}$ such that the maps $t \rightarrow \beta_{i}(t, x)$ belong to $L^{q}(I)$ for every $x \in \mathbb{R}^{d}$ and

$$
\sup _{(t, x) \in Q}\left|\tilde{\beta}_{1}(t, x)\right|<+\infty, \quad \int_{\mathbb{R}^{d}}\left(\int_{I}\left|\tilde{\beta}_{2}(t, x)\right|^{q} \mathrm{~d} t\right) \mathrm{d} x<+\infty .
$$

The map $B$ is then well defined, it belongs to $L^{q}(I \times F)$ for every $F$ with finite Lebesgue measure, and it is easy to check that $\partial_{t} B=\beta$.

Finally we have

$$
B(t, x)-B(s, x)=\kappa(x) B_{1}(s, t, x)+B_{2}(s, t, x), \quad B_{1}(s, t, x):=\int_{s}^{t} \tilde{\beta}_{1}(r, x) \mathrm{d} r, B_{2}(s, t, x):=\int_{s}^{t} \tilde{\beta}_{2}(r, x) \mathrm{d} r
$$

with $\left\|B_{1}(s, t, \cdot)\right\|_{L^{\infty}\left(\mathbb{R}^{d}\right)} \leq(t-s)\left\|\beta_{1}\right\|_{L^{\infty}(Q)}$ and $\left\|B_{2}(s, t, \cdot)\right\|_{L^{q}\left(\mathbb{R}^{d}\right)} \leq(t-s)^{1 / p}\left\|\beta_{2}\right\|_{L^{q}(Q)}$, which shows that

$$
\|B(t, \cdot)-B(s, \cdot)\|_{\mathcal{X}^{q}\left(\mathbb{R}^{d}\right)} \leq(t-s)^{1 / p}\|\beta\|_{\mathcal{X}^{q}(Q)} .
$$

Whenever $\alpha \in \mathcal{X}^{q}(Q)$, functions satisfying (5.1) enjoy the properties analyzed in the previous section.

Proposition 5.6 (Regularity and precise representatives of weak subsolutions) Let $u$ be a weak subsolution to (5.1) with $\alpha \in \mathcal{X}^{q}(Q)$ and let $\beta:=\alpha+\gamma_{H}^{-}, B$ as (4.23). Then $u, \beta$ satisfy the assumptions of Lemma 4.3 and Lemma 4.5 (with $\Omega:=\mathbb{R}^{d}$ ), in particular $u$ admits a precise representative $\mathrm{u}$ such that $t \mapsto \mathrm{u}(t, x)$ is right continuous for every $x \in \mathbb{R}^{d}, u=\mathrm{u} \lambda$-a.e. in $Q$ and (4.13) holds. Moreover, $u$ has traces $u_{0}^{+} \in L^{0}\left(\mathbb{R}^{d} ; \mathbb{R} \cup\{-\infty\}\right)$ and $u_{1}^{-} \in L^{0}\left(\mathbb{R}^{d} ; \mathbb{R} \cup\{+\infty\}\right)$ at $t=0$ and $t=1$ respectively, which are the pointwise limit of $\mathrm{u}(t, \cdot)$ as $t \downarrow 0$ and $t \uparrow 1$ in $L^{0}\left(\mathbb{R}^{d} ; \overline{\mathbb{R}}\right)$ and satisfy the properties of Lemma 4.5 .

Proof. Defining $\beta:=\alpha+\gamma_{H}^{-}$we immediately see that $-\partial_{t} u \leq \beta$ in $\mathscr{D}^{\prime}(Q)$ and we can apply Lemma 4.3 and 4.5, see also Remark 4.4

Motivated by the previous result we introduce the set $\operatorname{HJ}_{q}(Q, H)$ of pairs $(u, \alpha)$ solving (5.1), where the right-hand side belongs to $\mathcal{X}^{q}(Q)$.

Definition 5.7 (The convex set $\operatorname{HJ}_{q}(Q, H)$ ) We will denote by $\operatorname{HJ}_{q}(Q, H)$ the collection of pairs $(u, \alpha) \in$ $L_{\mathrm{loc}}^{1}(Q) \times \mathcal{X}^{q}(Q)$ solving (5.1). We will always use the symbol u to denote a precise representative of $u$ according to Proposition 5.6.

Since the Hamiltonian $H$ is convex with respect to its second variable, it is easy to check that the set $\mathrm{HJ}_{q}(Q, H)$ is a convex subset of $L_{\text {loc }}^{1}(Q) \times \mathcal{X}^{q}(Q)$.

\subsection{A priori estimates and stability for weak subsolutions}

We derive now some regularity properties and a priori estimates for weak subsolutions of Hamilton Jacobi equations, by studying their duality with suitable classes of solutions to the continuity equation, and in particular with Wasserstein geodesics. We will always denote by u a precise representative associated to a pair $(u, \alpha) \in \operatorname{HJ}_{q}(Q, H)$ and by $\mu$ the precise representative associated to a pair $(m, v) \in \mathrm{CE}_{2, p}(Q)$. We start with an estimate in the case that the measure $m$ is bounded and with compact support. 
Proposition 5.8 Let $(u, \alpha) \in \mathrm{HJ}_{q}(Q, H)$ and let $(m, \boldsymbol{v}) \in \mathrm{CE}_{2, \infty}(Q)$. We also suppose that there exists a compact set $K \subset \mathbb{R}^{d}$ such that $\operatorname{supp}\left(\mu_{t}\right) \subset K$ for every $t \in[0,1]$.

Setting $\beta:=\alpha+\gamma_{H}^{-}$, for every $0<s<t<1$ we have

$$
\int_{\mathbb{R}^{d}} \mathrm{u}_{t} \mathrm{~d} \mu_{t}-\int_{\mathbb{R}^{d}} \mathrm{u}_{s} \mathrm{~d} \mu_{s} \geq-\frac{c_{H}}{2} \int_{s}^{t} \int_{\mathbb{R}^{d}}|\boldsymbol{v}(r, x)|^{2} m(r, x) \mathrm{d} x \mathrm{~d} r-\int_{s}^{t} \int_{\mathbb{R}^{d}} \beta(r, x) m(r, x) \mathrm{d} x \mathrm{~d} r .
$$

Proof. We introduce a pair of convolution kernels $h, k$ as in (4.10), (4.30), (4.31), and we keep the notation of Lemma 4.6. setting $u_{\tau, \varepsilon}=u \star \eta_{\tau, \varepsilon}$ and choosing $\tau<1-t$.

Since by Remark $5.2 u$ is a weak subsolution to (5.3) with $\beta:=\alpha+\gamma_{H}^{-}$, then $u_{\tau, \varepsilon}$ is a classical subsolution to

$$
-\partial_{t} u_{\tau, \varepsilon}+\frac{1}{2 c_{H}}\left|D u_{\tau, \varepsilon}\right|^{2} \leq \beta_{\tau, \varepsilon}
$$

where $\beta_{\tau, \varepsilon}:=\beta \star \eta_{\tau, \varepsilon}$. Whence

$$
\begin{aligned}
\int_{\mathbb{R}^{d}} u_{\tau, \varepsilon}(t, x) \mathrm{d} \mu_{t}(x) & -\int_{\mathbb{R}^{d}} u_{\tau, \varepsilon}(s, x) \mathrm{d} \mu_{s}(x)=\int_{s}^{t} \int_{\mathbb{R}^{d}}\left(\partial_{t} u_{\tau, \varepsilon}+D u_{\tau, \varepsilon} \cdot \boldsymbol{v}\right) \mathrm{d} \mu_{r} \mathrm{~d} r \\
& \geq \int_{s}^{t}\left(\int_{\mathbb{R}^{d}}\left(\frac{1}{2 c_{H}}\left|D u_{\tau, \varepsilon}\right|^{2}+D u_{\tau, \varepsilon} \boldsymbol{v}-\beta_{\tau, \varepsilon}\right) \mathrm{d} \mu_{r}\right) \mathrm{d} r \\
& \geq-\frac{c_{H}}{2} \int_{s}^{t} \int_{\mathbb{R}^{d}}|\boldsymbol{v}|^{2} m \mathrm{~d} x \mathrm{~d} r-\int_{s}^{t} \int_{\mathbb{R}^{d}} \beta_{\tau, \varepsilon} m \mathrm{~d} x \mathrm{~d} r .
\end{aligned}
$$

We first pass to the limit as $\varepsilon \downarrow 0$ and then as $\tau \downarrow 0$. Standard results for convolutions (see also Lemma 4.6) imply that $\beta_{\tau, \varepsilon} \rightarrow \beta$ in $L_{\text {loc }}^{1}(Q)$, so that we can easily pass to the limit in (5.15).

Concerning the first term of (5.14), thanks to the compact support and the bounded density of $\mu_{t}$ and $\mu_{s}$, we can first use (4.37) and then (4.15) of Lemma 4.3 to pass to the limit, and deduce (5.12) thanks to the choice of the precise right continuous representative.

Corollary 5.9 Let us suppose that $(u, \alpha) \in \mathrm{HJ}_{q}(Q, H)$. For every pair of probability measures $\mu^{\prime}, \mu^{\prime \prime} \in$ $\mathcal{P}\left(\mathbb{R}^{d}\right)$ with compact support and $L^{\infty}$-densities $m^{\prime}, m^{\prime \prime}$, and for every $0<s<t<1$ we have

$$
\begin{aligned}
\int_{\mathbb{R}^{d}} \mathrm{u}_{t} \mathrm{~d} \mu^{\prime \prime}-\int_{\mathbb{R}^{d}} \mathrm{u}_{s} \mathrm{~d} \mu^{\prime} \geq & -\frac{c_{H}}{2(t-s)} W_{2}^{2}\left(\mu^{\prime}, \mu^{\prime \prime}\right) \\
& -(t-s)^{1 / p}\|\beta\|_{\mathcal{X}^{q}(Q)} \max \left(\left\|m^{\prime}\right\|_{L^{p} \cap L_{\kappa}^{1}\left(\mathbb{R}^{d}\right)},\left\|m^{\prime \prime}\right\|_{L^{p} \cap L_{\kappa}^{1}\left(\mathbb{R}^{d}\right)}\right),
\end{aligned}
$$

where $\beta:=\alpha+\gamma_{H}^{-}$.

Proof. Let $\left(\mu_{r}\right)_{r \in[s, t]}$ be the (rescaled) Wasserstein geodesic satisfying $\mu_{s}:=\mu^{\prime}$ and $\mu_{t}:=\mu^{\prime \prime}$. Then $\mu$ satisfies the continuity equation with minimal velocity field $\boldsymbol{v}$ satisfying

$$
\int_{s}^{t} \int_{\mathbb{R}^{d}}|\boldsymbol{v}|^{2} \mathrm{~d} \mu_{r} \mathrm{~d} r=\frac{1}{(t-s)} W_{2}^{2}\left(\mu^{\prime}, \mu^{\prime \prime}\right) .
$$

By writing the decomposition $\beta=\kappa \beta_{0}+\beta_{1}$ with $\beta_{0} \in L^{\infty}(Q)$ and $\beta_{1} \in L^{q}(Q)$ we get

$$
\begin{aligned}
\int_{(s, t) \times \mathbb{R}^{d}} \beta \mathrm{d} \tilde{\mu} & =\int_{(s, t) \times \mathbb{R}^{d}} \beta_{0} \kappa \mathrm{d} \tilde{\mu}+\int_{(s, t) \times \mathbb{R}^{d}} \beta_{1} \mathrm{~d} \tilde{\mu} \\
& \leq(t-s)\left\|\beta_{0}\right\|_{L^{\infty}(Q)} \max _{r \in[s, t]} \int_{\mathbb{R}^{d}} \kappa \mathrm{d} \mu_{r}+(t-s)^{1 / p}\left\|\beta_{1}\right\|_{L^{q}(Q)} \max _{r \in[s, t]}\left\|m_{r}\right\|_{L^{p}\left(\mathbb{R}^{d}\right)} \\
& \leq(t-s)^{1 / p}\left(\left\|\beta_{0}\right\|_{L^{\infty}(Q)}+\left\|\beta_{1}\right\|_{L^{q}(Q)}\right) \max \left(\left\|m^{\prime}\right\|_{L^{p} \cap L_{\kappa}^{1}\left(\mathbb{R}^{d}\right)},\left\|m^{\prime \prime}\right\|_{L^{p} \cap L_{\kappa}^{1}\left(\mathbb{R}^{d}\right)}\right) .
\end{aligned}
$$

Therefore, the conclusion follows from Proposition 5.8

Theorem 5.10 (Interior regularity and traces of precise representatives) If $(u, \alpha) \in \mathrm{HJ}_{q}(Q, H)$ then the precise representatives $\mathrm{u}_{t}=\mathrm{u}(t, \cdot)$ belong to $\mathcal{X}^{q}\left(\mathbb{R}^{d}\right)$ for every $t \in(0,1)$. For every $\mu=m \mathscr{L}^{d} \in \mathcal{P}_{2, p}^{r}\left(\mathbb{R}^{d}\right)$ the map $t \mapsto \int_{\mathbb{R}^{d}} \mathrm{u}(t, x) \mathrm{d} \mu$ (respectively $t \mapsto \int_{\mathbb{R}^{d}} \mathrm{u}^{-}(t, x) \mathrm{d} \mu$ ) is right (respectively left) continuous in $I$, $u^{-}(1, \cdot), u^{+}(0, \cdot)$ are semiintegrable with respect to $\mu$ and

$$
\begin{aligned}
& \exists \lim _{t \uparrow 1} \int_{\mathbb{R}^{d}} \mathrm{u}(t, x) \mathrm{d} \mu=\int_{\mathbb{R}^{d}} u^{-}(1, x) \mathrm{d} \mu \quad \in(-\infty,+\infty] ; \\
& \exists \lim _{t \downarrow 0} \int_{\mathbb{R}^{d}} \mathrm{u}(t, x) \mathrm{d} \mu=\int_{\mathbb{R}^{d}} u^{+}(0, x) \mathrm{d} \mu \quad \in[-\infty,+\infty) .
\end{aligned}
$$


Moreover, for every $0 \leq a<r<b \leq 1$ and every pair of nonnegative measures $\mu_{a, b}=m_{a, b} \mathscr{L}^{d} \in \mathcal{P}_{2, p}^{r}\left(\mathbb{R}^{d}\right)$ such that $\mathrm{u}_{a} \in L^{1}\left(\mu_{a}\right), \mathrm{u}_{b} \in L^{1}\left(\mu_{b}\right)$ with $I_{a}=\int_{\mathbb{R}^{d}} \mathbf{u}_{a} \mathrm{~d} \mu_{a}, I_{b}:=\int_{\mathbb{R}^{d}} \mathbf{u}_{b} \mathrm{~d} \mu_{b}$ we have

$$
\left\|\mathbf{u}_{r}\right\|_{\mathcal{X}^{q}\left(\mathbb{R}^{d}\right)} \leq\left(I_{a}\right)_{-}+\left(I_{b}\right)_{+}+\left(\frac{c_{H}(b-a)}{(b-r)(r-a)}+2\|\beta\|_{\mathcal{X}^{q}(Q)}\right) \max \left(\left\|m_{a}\right\|_{L^{p} \cap L_{\kappa}^{1}\left(\mathbb{R}^{d}\right)},\left\|m_{b}\right\|_{L^{p} \cap L_{\kappa}^{1}\left(\mathbb{R}^{d}\right)}\right) .
$$

Proof. Let us first choose $0<a<b<1, r \in(a, b), \mu_{a, b}=m_{a, b} \mathscr{L}^{d} \in \mathcal{P}_{2}\left(\mathbb{R}^{d}\right), m_{a, b} \in L^{p} \cap L_{\kappa}^{1}\left(\mathbb{R}^{d}\right)$ such that $\mathrm{u}_{a} \in L^{1}\left(\mu_{a}\right), \mathbf{u}_{b} \in L^{1}\left(\mu_{b}\right)$; since $\mathbf{u}_{a}, \mathbf{u}_{b}$ belong to $L_{\text {loc }}^{1}\left(\mathbb{R}^{d}\right)$ the collection of such measures is surely not empty.

For $R, K>0$ sufficiently big we consider the probability measures $\mu_{b, R, K}=m_{b, R, K} \mathscr{L}^{d} \in \mathcal{P}_{2}^{r}\left(\mathbb{R}^{d}\right)$

$$
m_{b, R, K}:=c_{R, K}\left(m_{b} \wedge K\right) \chi_{B_{R}(0)} \mathscr{L}^{d}, \quad c_{R, K}:=\left(\int_{B_{R}(0)}\left(m_{b} \wedge K\right) \mathrm{d} x\right)^{-1} \in[1,+\infty),
$$

where the truncation is needed in order to apply Corollary 5.9, We choose a bounded measurable $\varphi: \mathbb{R}^{d} \rightarrow$ $[0, \infty)$ with compact support such that $\|\varphi\|_{L^{1}\left(\mathbb{R}^{d}\right)}>0$; we can apply (5.16) with the choices $s:=r, t:=b$, $m^{\prime}=\frac{\varphi(x) \mathscr{L}^{d}}{\|\varphi\|_{L^{1}\left(\mathbb{R}^{d}\right)}}, m^{\prime \prime}=m_{b, R, K}$. The triangle inequality for $W_{2}$ and the fact that $W_{2}^{2}\left(\mu, \delta_{0}\right)=\int_{\mathbb{R}^{d}}|x|^{2} \mathrm{~d} \mu$ yield

$$
W_{2}^{2}\left(\mu^{\prime}, \mu^{\prime \prime}\right) \leq 2\left(\int_{\mathbb{R}^{d}}|x|^{2} \mathrm{~d} \mu^{\prime}(x)+c_{R, K} \int_{\mathbb{R}^{d}}|x|^{2} \mathrm{~d} \mu_{b}\right)
$$

on the other hand

$$
\left\|m^{\prime}\right\|_{L^{p} \cap L_{\kappa}^{1}\left(\mathbb{R}^{d}\right)} \leq \frac{1}{\|\varphi\|_{L^{1}\left(\mathbb{R}^{d}\right)}}\|\varphi\|_{L^{p} \cap L_{\kappa}^{1}\left(\mathbb{R}^{d}\right)}, \quad\left\|m^{\prime \prime}\right\|_{L^{p} \cap L_{\kappa}^{1}\left(\mathbb{R}^{d}\right)} \leq c_{R, K}\left\|m_{b}\right\|_{L^{p} \cap L_{\kappa}^{1}\left(\mathbb{R}^{d}\right)}
$$

so that 5.16 yields

$$
\begin{aligned}
\int_{\mathbb{R}^{d}} \mathrm{u}(r, x) \varphi(x) \mathrm{d} x \leq & \|\varphi\|_{L^{1}\left(\mathbb{R}^{d}\right)} \int_{\mathbb{R}^{d}} \mathrm{u}_{b} \mathrm{~d} \mu_{b, R, K}+\frac{c_{H}}{(b-r)} c_{R, K}\|\varphi\|_{L_{\kappa}^{1}\left(\mathbb{R}^{d}\right)}\left\|m_{b}\right\|_{L_{\kappa}^{1}\left(\mathbb{R}^{d}\right)} \\
& +c_{R, K}\|\beta\|_{\mathcal{X}^{q}(Q)}\|\varphi\|_{L^{p} \cap L_{\kappa}^{1}\left(\mathbb{R}^{d}\right)}\left\|m_{b}\right\|_{L^{p} \cap L_{\kappa}^{1}\left(\mathbb{R}^{d}\right)} .
\end{aligned}
$$

We can now pass to the limit as $R, K \uparrow \infty$ observing that $c_{R, K} \rightarrow 1$, obtaining

$$
\int_{\mathbb{R}^{d}} \mathrm{u}(r, x) \varphi(x) \mathrm{d} x \leq C_{b}\|\varphi\|_{L^{p} \cap L_{\kappa}^{1}\left(\mathbb{R}^{d}\right)}, \quad C_{b}:=\left(I_{b}\right)_{+}+\left(\frac{c_{H}}{(b-r)}+\|\beta\|_{\mathcal{X}^{q}(Q)}\right)\left\|m_{b}\right\|_{L^{p} \cap L_{\kappa}^{1}\left(\mathbb{R}^{d}\right)} .
$$

Replacing $\varphi$ with $\varphi \chi_{\{u(r, \cdot)>0\}}$ we see that the same estimate holds for the positive part of $u$; (4.46) then yields

$$
\|\mathrm{u}(r, \cdot) \vee 0\|_{\mathcal{X}^{q}\left(\mathbb{R}^{d}\right)} \leq C_{b} .
$$

A similar argument, replacing now $b$ with $a$ yields

$$
\int_{\mathbb{R}^{d}} \mathrm{u}(r, x) \varphi(x) \mathrm{d} x \geq-C_{a}\|\varphi\|_{L^{p} \cap L_{\kappa}^{1}\left(\mathbb{R}^{d}\right)}, \quad C_{a}:=\left(I_{a}\right)_{-}+\left(\frac{c_{H}}{(r-a)}+\|\beta\|_{\mathcal{X}^{q}(Q)}\right)\left\|m_{a}\right\|_{L^{p} \cap L_{\kappa}^{1}\left(\mathbb{R}^{d}\right)} .
$$

We deduce that $\|\mathrm{u}(r, \cdot) \wedge 0\|_{\mathcal{X}^{q}(Q)} \leq C_{a}$ and

$$
\|\mathrm{u}(r, \cdot)\|_{\mathcal{X}^{q}\left(\mathbb{R}^{d}\right)} \leq C_{a}+C_{b}
$$

which yields (5.18).

Since $\mathcal{X}^{q}\left(\mathbb{R}^{d}\right)$ is the dual space of $L^{p} \cap L_{\kappa}^{1}\left(\mathbb{R}^{d}\right)$ (see Lemma 4.8), the right continuity of the curves $t \mapsto \int_{\mathbb{R}^{d}} \mathrm{u}_{t} \mathrm{~d} \mu$ for every $\mu=m \mathscr{L}^{d} \in \mathcal{P}_{2}\left(\mathbb{R}^{d}\right)$ with $m \in L^{p}\left(\mathbb{R}^{d}\right)$ is equivalent to the right continuity of $t \mapsto \mathrm{u}_{t}$ with respect to the weak* topology of $\mathcal{X}^{q}\left(\mathbb{R}^{d}\right)$. Thanks to the uniform bound (5.18) and the strong density of bounded functions with compact support in $L^{p} \cap L_{\kappa}^{1}\left(\mathbb{R}^{d}\right)$, this property follows by the right continuity in $L^{0}\left(\mathbb{R}^{d}\right)$.

Finally, (5.17) follows by decomposing $u$ as $w-B$ as in Proposition [5.6 by Lemma 5.5] it is sufficient to study the behaviour of $w$. By monotonicity, since the right trace $w^{-}(1, \cdot) \geq \mathrm{w}(r, \cdot)$ for every $r<1$ and $\mathrm{w}(r, \cdot) \in \mathcal{X}^{q}\left(\mathbb{R}^{d}\right)$, we immediately get that the negative part of $w^{-}(1, \cdot)$ is $\mu$-integrable. The first property of (5.17) then follows by Beppo Levi's Monotone Convergence Theorem. A similar argument works for $u^{+}(0, \cdot)$.

Eventually passing to the limit in (5.18) as $b \uparrow 1$ and $a \downarrow 0$, we extend its validity to the case $a=0$ and $b=1$.

We conclude this section with a stability argument for the weak subsolutions of (5.1). 
Theorem 5.11 (Stability and semicontinuity) Suppose that the sequence $\left(u_{n}, \alpha_{n}\right) \in \mathrm{HJ}_{q}(Q, H), n \in \mathbb{N}$, satisfies the local uniform bound

$$
\sup _{n \in \mathbb{N}} \sup _{a \leq t \leq b}\left\|\mathrm{u}_{n}(t, \cdot)\right\|_{\mathcal{X}^{q}\left(\mathbb{R}^{d}\right)}<\infty \quad \text { for every } 0<a<b<1
$$

and that $\alpha_{n} \rightarrow^{*} \alpha$ weakly* in $\mathcal{X}^{q}(Q)$. Then there exist a subsequence $k \mapsto n(k)$ and a limit function $u \in L_{\mathrm{loc}}^{1}(Q)$ such that

(a) $(u, \alpha) \in \operatorname{HJ}_{q}(Q, H)$ (thus in particular $D u \in L_{\text {loc }}^{2}\left(Q ; \mathbb{R}^{d}\right)$ );

(b)

$$
\begin{array}{rlr}
\mathbf{u}_{n(k)}(t, \cdot) \rightarrow^{*} \tilde{u} & \text { in } \mathcal{X}^{q}\left(\mathbb{R}^{d}\right) & \text { with } \mathbf{u}^{-}(t, \cdot) \leq \tilde{\mathbf{u}}_{t} \leq \mathrm{u}(t, \cdot) \text { for every } t \in(0,1), \\
u_{n(k)} \rightarrow u & \text { in } L^{q}(K) & \text { for all compact sets } K \subset Q \\
D u_{n(k)} \rightarrow D u & \text { in } L^{2}\left(K ; \mathbb{R}^{d}\right) & \text { for all compact sets } K \subset Q .
\end{array}
$$

(c) For every $\mu_{i}=m_{i} \mathscr{L}^{d}$ with $m_{i} \in L^{p} \cap L_{\kappa}^{1}\left(\mathbb{R}^{d}\right)$ the quantities

$$
\mathcal{A}\left(u_{n}, \alpha_{n}\right):=\int_{\mathbb{R}^{d}} u_{n}^{+}(0, \cdot) \mathrm{d} \mu_{0}-\int_{\mathbb{R}^{d}} u_{n}^{-}(1, \cdot) \mathrm{d} \mu_{1}-\int_{Q} F^{*}\left(x, \alpha_{n}\right) \mathrm{d} x \mathrm{~d} t
$$

and the corresponding one $\mathcal{A}(u, \alpha)$ defined on the limit pair $(u, \alpha)$ are well defined in $[-\infty,+\infty)$ and satisfy

$$
\limsup _{n \uparrow+\infty} \mathcal{A}\left(u_{n}, \alpha_{n}\right) \leq \mathcal{A}(u, \alpha)
$$

Proof. (b) Let $\beta_{n}:=\alpha_{n}+\gamma_{H}^{-}$, let $B_{n}$ be correspondly defined as in Lemma 5.5 and let $\mathrm{w}_{n}:=\mathrm{u}_{n}+B_{n}$, so that $\mathrm{w}_{n}$ is increasing w.r.t. time and right continuous.

It is easy to see that $B_{n}$ satisfies (5.26), so that it is sufficient to prove this convergence property for $\mathrm{w}_{n}$, which still obeys to

$$
\sup _{a \leq t \leq b}\left\|\mathrm{w}_{n}(t, \cdot)\right\|_{\mathcal{X}^{q}\left(\mathbb{R}^{d}\right)} \leq C(a, b)<\infty \quad \text { for every } 0<a<b<1, n \in \mathbb{N} .
$$

We fix an interval $[a, b] \subset(0,1)$ and we consider the set $K:=\left\{z \in \mathcal{X}^{q}\left(\mathbb{R}^{d}\right):\|z\|_{\mathcal{X}^{q}\left(\mathbb{R}^{d}\right)} \leq C(a, b)\right\}$ endowed with the weak*-topology $\sigma$ of $\mathcal{X}^{q}\left(\mathbb{R}^{d}\right)$. $K$ is compact w.r.t. $\sigma$. We introduce the distance

$$
\delta\left(z_{1}, z_{2}\right):=\int_{\mathbb{R}^{d}}\left|z_{1}(x)-z_{2}(x)\right| \rho(x) \mathrm{d} x \quad \text { for every } z_{1}, z_{2} \in K,
$$

where $\rho(x)$ is the density of the measure $\varrho$ we used to define the distance $d$ in $L^{0}\left(\mathbb{R}^{d}\right)$, see (4.3). Clearly $\delta \geq d$ and it is also $\sigma$ lower semicontinuous, since the weak* topology of $\mathcal{X}^{q}\left(\mathbb{R}^{d}\right)$ is clearly stronger than the weak topology in $L^{1}\left(\mathbb{R}^{d}, \varrho\right)$.

For every $a \leq s \leq t \leq b$ we have

$$
\begin{aligned}
\delta\left(\mathrm{w}_{n}(s), \mathrm{w}_{n}(t)\right) & \leq \int_{\mathbb{R}^{d}}\left|\mathrm{w}_{n}(t, x)-\mathrm{w}_{n}(s, x)\right| \mathrm{d} \varrho(x)=\int_{\mathbb{R}^{d}}\left(\mathrm{w}_{n}(t, x)-\mathrm{w}_{n}(s, x)\right) \mathrm{d} \varrho(x) \\
& =\int_{\mathbb{R}^{d}} \mathrm{w}_{n}(t, x) \mathrm{d} \varrho(x)-\int_{\mathbb{R}^{d}} \mathrm{w}_{n}(s, x) d \varrho(x)
\end{aligned}
$$

so that the $\delta$-total variation of $\mathrm{w}_{n}$ in the interval $[a, b]$ can be estimated by

$$
\operatorname{Var}_{\delta}\left(\mathrm{w}_{n} ;[a, b]\right) \leq \int_{\mathbb{R}^{d}} \mathrm{w}_{n}(b, x) \mathrm{d} \varrho(x)-\int_{\mathbb{R}^{d}} \mathrm{w}_{n}(a, x) \mathrm{d} \varrho(x) \leq C(a, b)\|\rho\|_{L^{p} \cap L_{\kappa}^{1}\left(\mathbb{R}^{d}\right)}, \quad \varrho=\rho \mathscr{L}^{d} .
$$

Since the sequence of functions $\mathrm{w}_{n}$ takes value in the $\sigma$-compact set $K$ and their $\delta$-total variation is uniformly

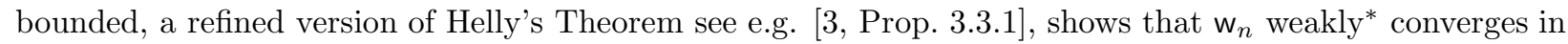
$\mathcal{X}^{q}\left(\mathbb{R}^{d}\right)$, for every $t \in[a, b]$, to a function $\tilde{\mathrm{w}}$. A standard diagonal argument yields the same convergence for every $t \in I$. Since $t \mapsto \tilde{\mathrm{w}}_{t}$ is increasing, we can set $\mathrm{w}_{t}:=\lim _{s \downarrow t} \tilde{\mathrm{w}}_{s}$ thus obtaining (5.26). Notice that if $J$ denotes the (at most) countable set $J:=\left\{t \in(0,1): \mathrm{w}_{t}^{-} \neq \mathrm{w}_{t}\right\}$, we have $\tilde{\mathrm{w}}_{t}=\mathrm{w}_{t}$ for every $t \in(0,1) \backslash J$. 
If $\zeta \in L^{p}(Q)$ has compact support contained in $[a, b] \times F$, where $F$ is a compact subset of $\mathbb{R}^{d}$, we get

$$
\begin{aligned}
\lim _{n \rightarrow \infty} \int_{Q} \mathrm{w}_{n} \zeta \mathrm{d} t \mathrm{~d} x & =\lim _{n \rightarrow \infty} \int_{a}^{b}\left(\int_{F} \mathrm{w}_{n}(t, x) \zeta(t, x) \mathrm{d} x\right) \mathrm{d} t=\int_{a}^{b}\left(\lim _{n \rightarrow \infty} \int_{F} \mathrm{w}_{n}(t, x) \zeta(t, x) \mathrm{d} x\right) \mathrm{d} t \\
& =\int_{a}^{b}\left(\int_{F} \mathrm{w}(t, x) \zeta(t, x) \mathrm{d} x\right) \mathrm{d} t
\end{aligned}
$$

where we have applied the above convergence result and the Lebesgue Dominated Convergence Theorem; notice that for a.e. $t \in[a, b]$

$$
\left|\int_{F} \mathrm{w}_{n}(t, x) \zeta(t, x) \mathrm{d} x\right| \leq C(a, b) g(t), \quad g(t):=\|\zeta(t, \cdot)\|_{L^{p} \cap L_{\kappa}^{1}\left(\mathbb{R}^{d}\right)} \leq\|\zeta(t, \cdot)\|_{L^{p}\left(\mathbb{R}^{d}\right)}+\|\zeta(t, \cdot)\|_{L_{\kappa}^{1}\left(\mathbb{R}^{d}\right)}
$$

so that $\int_{a}^{b} g(t) \mathrm{d} t \leq\|\zeta\|_{L^{p}(Q)}+\|\zeta\|_{L_{\kappa}^{1}(Q)}$.

Concerning (5.28), it is sufficient to show that for every compact set $K \subset Q$ there exists a constant $C(K)$ such that

$$
\int_{K}\left|D u_{n}\right|^{2} \mathrm{~d} x \mathrm{~d} t \leq C(K) \quad \text { for every } n \in \mathbb{N} .
$$

We select a nonnegative function $\xi \in C_{c}^{\infty}(Q)$ such that $\xi \equiv 1$ on $K$; in particular the support of $\xi$ will be contained in $[a, b] \times \mathbb{R}^{d}$ for some interval $[a, b] \subset(0,1)$. The distributional formulation (5.2) of (5.1) yields

$$
\begin{aligned}
\int_{K}\left|D u_{n}\right|^{2} \mathrm{~d} x \mathrm{~d} t & \leq \int_{Q}\left|D u_{n}\right|^{2} \xi \mathrm{d} x \mathrm{~d} t \leq \int_{Q}\left(\alpha_{n} \xi+u_{n} \partial_{t} \xi\right) \mathrm{d} x \mathrm{~d} t \\
& \leq\left(\sup _{n \in \mathbb{N}}\left\|\alpha_{n}\right\|_{\mathcal{X}^{q}}\right)\|\xi\|_{L^{p} \cap L_{\kappa}^{1}(Q)}+\left(\sup _{n \in \mathbb{N}} \sup _{t \in[a, b]}\left\|u_{n}(t, \cdot)\right\| \mathcal{X}^{q}\right)\left\|\partial_{t} \xi\right\|_{L^{p} \cap L_{\kappa}^{1}(Q)},
\end{aligned}
$$

which yields (5.34).

(a) For every nonnegative test function $\xi \in C_{c}^{\infty}(Q)$ we have to pass to the limit in the inequality (5.2) written for $u_{n}, \alpha_{n}$. By the previous claim, it is sufficient to prove that

$$
\liminf _{n \rightarrow \infty} \int_{Q} H\left(x, D u_{n}\right) \xi \mathrm{d} x \mathrm{~d} t \geq \int_{Q} H(x, D u) \xi \mathrm{d} x .
$$

This property follows by the weak $L^{2}$ lower semicontinuity of the integral functional associated to $H$ (and weighted by $\xi$ ), relying on the structural properties (convexity and lower bound) of $H$ stated in Assumptions 2.1(H3), see e.g. [17, Theorem 6.54].

(c) (5.29) is well defined (possibly taking the value $-\infty$ ) thanks to Theorem 5.10 and the fact that all the three terms belong to $[-\infty,+\infty)$.

Since $F^{*}$ is nonnegative, measurable, convex and lower semicontinuous w.r.t. its second argument, the last integral functional $\int_{Q} F^{*}(x, \alpha) \mathrm{d} x \mathrm{~d} t$ is lower semicontinuous with respect to weak $L^{1}$ convergence on compact subsets, so that

$$
\limsup _{n \rightarrow \infty}-\int_{Q} F^{*}\left(x, \alpha_{n}\right) \mathrm{d} x \mathrm{~d} t \leq-\int_{Q} F^{*}(x, \alpha) \mathrm{d} x \mathrm{~d} t .
$$

Let us now consider the behaviour of the first integral defining $\mathcal{A}\left(u_{n}, \alpha_{n}\right)$. In order to prove inequality (5.30) we observe that for $\varepsilon>0$

$$
u_{n}^{+}(0, x)=w_{n}^{+}(0, x) \leq \mathrm{w}_{n}(\varepsilon, x)=\mathrm{u}_{n}(\varepsilon, x)+B_{n}(\varepsilon, x)
$$

and, by (5.11),

so that

$$
\left|\int_{\mathbb{R}^{d}} B_{n}(\varepsilon, x) \mathrm{d} \mu_{0}(x)\right| \leq(t-s)\left\|\beta_{n}\right\|_{\mathcal{X}^{q}(Q)}\left\|m_{0}\right\|_{L^{p} \cap L_{\kappa}^{1}\left(\mathbb{R}^{d}\right)},
$$

$$
\int_{\mathbb{R}^{d}} u_{n}^{+}(0, x) \mathrm{d} \mu_{0} \leq \int_{\mathbb{R}^{d}} \mathrm{u}_{n}(\varepsilon, x) \mathrm{d} \mu_{0}+o(1), \quad \text { as } \varepsilon \downarrow 0 .
$$

where $o(1)$ is uniform with respect to $n$. Sending $n \rightarrow \infty$, thanks to the weak* convergence of $\mathbf{u}_{n}(\varepsilon, \cdot)$ in $\mathcal{X}^{q}\left(\mathbb{R}^{d}\right)$, we get

$$
\limsup _{n \rightarrow \infty} \int_{\mathbb{R}^{d}} u_{n}^{+}(0, x) \mathrm{d} \mu_{0} \leq \limsup _{n \rightarrow \infty} \int_{\mathbb{R}^{d}} \mathrm{u}_{n}(\varepsilon, x) \mathrm{d} \mu_{0}+o(1)=\int_{\mathbb{R}^{d}} \mathrm{u}(\varepsilon, x) \mathrm{d} \mu_{0}+o(1) .
$$

Thanks to Theorem 5.10, passing to the limit as $\varepsilon \downarrow 0$ we get the first part of (5.30). A similar argument yields

$$
\liminf _{n \rightarrow \infty} \int_{\mathbb{R}^{d}} u_{n}^{-}(1, x) \mathrm{d} \mu_{1} \geq \int_{\mathbb{R}^{d}} u^{-}(1, x) \mathrm{d} \mu_{1} .
$$




\subsection{A general duality-transport result}

The following result is fundamental for the development of a variational approach to MFPPs. We derive a transport and a duality relation between weak subsolutions to (5.1) with $\alpha \in \mathcal{X}^{q}(Q)$ and $L^{p}$-solutions to the continuity equation.

In the next Theorem we will use an arbitrary test function $\zeta$ and its primitive $Z$ in $\mathscr{Z}_{c}$, see (4.11).

Theorem 5.12 Let $(u, \alpha) \in \operatorname{HJ}_{q}(Q, H)$ be a weak subsolution to (5.1) according to Definition 5.7 and let $(m, \boldsymbol{v}) \in \mathrm{CE}_{2, p}(Q)$ be a distributional solution to the continuity equation according to Definition [3.3. Then we have:

(1) for every $0<s<t<1$

$$
\int_{s}^{t} \int_{\mathbb{R}^{d}}|D u(r, x)|^{2} m(r, x) \mathrm{d} x \mathrm{~d} r<+\infty
$$

(2) for every pair $(\zeta, Z) \in \mathscr{Z}_{c}$

$$
\partial_{t}(Z(u) m)+\nabla \cdot(Z(u) m \boldsymbol{v})+\zeta(u)(\alpha-H(x, D u)-D u \cdot \boldsymbol{v}) m \geq 0 \quad \text { in } \mathscr{D}^{\prime}(Q),
$$

also in duality with nonnegative functions $\varphi \in C_{c}^{1}(\bar{Q})$ if $\mu_{0}, \mu_{1} \ll \mathscr{L}^{d}$ :

$$
\begin{array}{r}
-\int_{Q} Z(u) m\left(\partial_{t} \varphi+D \varphi \cdot \boldsymbol{v}\right) \mathrm{d} x \mathrm{~d} t+\int_{Q} \zeta(u)(\alpha-H(x, D u)-D u \cdot \boldsymbol{v}) m \varphi \mathrm{d} x \mathrm{~d} t \\
\geq \int_{\mathbb{R}^{d}} Z\left(u^{+}(0, x)\right) \varphi(0, x) \mathrm{d} \mu_{0}(x)-\int_{\mathbb{R}^{d}} Z\left(u^{-}(1, x)\right) \varphi(1, x) \mathrm{d} \mu_{1}(x),
\end{array}
$$

(3) for every $s, t \in D_{p}[\mu]$ with $0<s<t<1$ it holds

$$
\int_{\mathbb{R}^{d}} \mathrm{u}_{s} \mathrm{~d} \mu_{s}-\int_{\mathbb{R}^{d}} \mathrm{u}_{t} \mathrm{~d} \mu_{t} \leq \int_{s}^{t} \int_{\mathbb{R}^{d}}(\alpha-H(x, D u)-D u \cdot \boldsymbol{v}) m \mathrm{~d} x \mathrm{~d} r .
$$

Moreover, (5.38) and (5.41) also hold for $s=0$ (respectively, $t=1$ ) provided $0 \in D_{p}[\mu]$ and $\int_{\mathbb{R}^{d}} u_{0}^{+} \mathrm{d} \mu_{0}>-\infty$ (respectively, $\left.1 \in D_{p}[\mu], \int_{\mathbb{R}^{d}} u_{1}^{-} \mathrm{d} \mu_{1}<+\infty\right)$.

Proof. Step 1. Let us first suppose that $u$ is bounded. By Theorem 5.10 the traces $u_{1}^{-}$and $u_{0}^{+}$are well defined and belong to $L^{\infty}\left(\mathbb{R}^{d}\right)$.

Operating a regularization by convolution according to the notation of Section 4.3, we know that $u_{\tau, \varepsilon}$ is a classical subsolution (with uniformly bounded derivatives) to

$$
-\partial_{t} u_{\tau, \varepsilon}+\tilde{H}_{\tau, \varepsilon} \leq \alpha_{\tau, \varepsilon} \text { in }[0,1-\tau] \times \mathbb{R}^{d}
$$

where $\tilde{H}_{\tau, \varepsilon}(x):=(H(x, D u)) * \eta_{\tau, \varepsilon}$. Similarly, by multiplying the previous inequality by $\zeta\left(u_{\tau, \varepsilon}\right)$, we also obtain

$$
\partial_{t} Z\left(u_{\tau, \varepsilon}\right)+\zeta\left(u_{\tau, \varepsilon}\right)\left(\alpha_{\tau, \varepsilon}-\tilde{H}_{\tau, \varepsilon}\right) \geq 0 \text { in }[0,1-\tau] \times \mathbb{R}^{d} .
$$

By selecting a nonnegative function $\varphi \in C_{c}^{\infty}\left([0,1-\tau) \times \mathbb{R}^{d}\right)$ and testing the continuity equation for $(m, \boldsymbol{v})$ with $\varphi \cdot Z\left(u_{\varepsilon, \tau}\right)$, we get for $\theta_{\tau, \varepsilon}:=\alpha_{\tau, \varepsilon}-\tilde{H}_{\tau, \varepsilon}-D u_{\tau, \varepsilon} \cdot \boldsymbol{v}$

$$
\begin{array}{rl}
\int_{\mathbb{R}^{d}} & Z\left(u_{\varepsilon, \tau}(0, x)\right) \varphi(0, x) m_{0}(x) \mathrm{d} x=-\int_{Q}\left(\partial_{t}\left(Z\left(u_{\varepsilon, \tau}\right) \varphi\right)+D\left(Z\left(u_{\varepsilon, \tau}\right) \varphi\right) \cdot \boldsymbol{v}\right) m \mathrm{~d} x \mathrm{~d} t \\
= & -\int_{Q} Z\left(u_{\varepsilon, \tau}\right) m\left(\partial_{t} \varphi+D \varphi \cdot \boldsymbol{v}\right) \mathrm{d} x \mathrm{~d} t-\int_{Q}\left(\partial_{t} Z\left(u_{\varepsilon, \tau}\right)+D Z\left(u_{\varepsilon, \tau}\right) \cdot \boldsymbol{v}\right) \varphi m \mathrm{~d} x \mathrm{~d} t \\
& \leq-\int_{Q} Z\left(u_{\varepsilon, \tau}\right) m\left(\partial_{t} \varphi+D \varphi \cdot \boldsymbol{v}\right) \mathrm{d} x \mathrm{~d} t+\int_{Q} \zeta\left(u_{\varepsilon, \tau}\right) \theta_{\tau, \varepsilon} m \varphi \mathrm{d} x \mathrm{~d} t
\end{array}
$$

Similarly, using the distributional formulation of the continuity equation we get for every $0 \leq s<t \leq 1-\tau$

$$
\begin{aligned}
0 & =\int_{\mathbb{R}^{d}} u_{\tau, \varepsilon}(s, \cdot) \mathrm{d} \mu_{s}-\int_{\mathbb{R}^{d}} u_{\tau, \varepsilon}(t, \cdot) \mathrm{d} \mu_{t}+\int_{s}^{t} \int_{\mathbb{R}^{d}}\left(\partial_{t} u_{\tau, \varepsilon}+D u_{\tau, \varepsilon} \cdot \boldsymbol{v}\right) \mathrm{d} \mu_{r} \mathrm{~d} r \\
& \geq \int_{\mathbb{R}^{d}} u_{\tau, \varepsilon}(s, \cdot) \mathrm{d} \mu_{s}-\int_{\mathbb{R}^{d}} u_{\tau, \varepsilon}(t, \cdot) \mathrm{d} \mu_{t}+\int_{s}^{t} \int_{\mathbb{R}^{d}}\left(\tilde{H}_{\tau, \varepsilon}+D u_{\tau, \varepsilon} \cdot \boldsymbol{v}-\alpha_{\tau, \varepsilon}\right) m d x \mathrm{~d} r
\end{aligned}
$$


Due to the growth condition from below which is assumed for $H$ (see (2.13)), we have

$$
\tilde{H}_{\tau, \varepsilon} \geq\left(\frac{1}{2 c_{H}}|D u|^{2}-\gamma_{H}^{-}\right) * \eta_{\tau, \varepsilon} \geq \frac{1}{2 c_{H}}\left|D u_{\tau, \varepsilon}\right|^{2}-\gamma_{H, \varepsilon}^{-}, \quad \gamma_{H, \varepsilon}^{-}(x):=\left(\gamma_{H}^{-} * \eta_{\tau, \varepsilon}\right)(x)=\left(\gamma_{H}^{-} * k_{\varepsilon}\right)(x) .
$$

Hence we estimate

$$
\tilde{H}_{\tau, \varepsilon}+D u_{\tau, \varepsilon} \cdot \boldsymbol{v} \geq \frac{1}{4 c_{H}}\left|D u_{\tau, \varepsilon}\right|^{2}-\gamma_{H, \varepsilon}^{-}-c_{H}|\boldsymbol{v}|^{2} \geq-\gamma_{H, \varepsilon}^{-}-c_{H}|\boldsymbol{v}|^{2}
$$

If we choose $s, t \in D_{p}[\mu]$ we can pass to the limit first as $\varepsilon \downarrow 0$ and then as $\tau \downarrow 0$ in (5.45), by invoking Fatou's Lemma and the lower bound (5.46) for the integral involving $\tilde{H}_{\tau, \varepsilon}+D u_{\tau, \varepsilon} \cdot \boldsymbol{v}$, standard convolution estimates for $\alpha_{\tau, \varepsilon}$ in $\mathcal{X}^{q}(Q)$, and Lemma 4.6 for the first two integrals; we thus obtain

$$
\int_{\mathbb{R}^{d}} \mathrm{u}(s, x) \mathrm{d} \mu_{s}(x)-\int_{\mathbb{R}^{d}} \mathrm{u}(t, x) \mathrm{d} \mu_{t}(x) \leq \int_{s}^{t} \int_{\mathbb{R}^{d}}(\alpha-H(x, D u)-D u \cdot \boldsymbol{v}) m \mathrm{~d} x \mathrm{~d} t
$$

for every $s, t \in[0,1) \cap D_{p}[\mu], s<t$. The very same argument, using the convolution kernel $\hat{h}(t):=h(-t)$ and choosing a point $s_{1}>s$ where $u_{s_{1}}^{-}=u_{s_{1}}$, yields the inequality (5.47) for $t=1$ with $s=s_{1}$. Adding the same inequality between $s$ and $s_{1}$ we conclude the proof of (5.41).

(5.47) and the lower bound $H(x, D u)-D u \cdot \boldsymbol{v} \geq \frac{1}{4 c_{H}}|D u|^{2}-\gamma_{H}^{-}-c_{H}|\boldsymbol{v}|^{2}$ yields

$$
\frac{1}{4 c_{H}} \int_{s}^{t} \int_{\mathbb{R}^{d}}|D u(r, x)|^{2} \mathrm{~d} \mu_{r} \mathrm{~d} r \leq \int_{\mathbb{R}^{d}} \mathrm{u}(t, \cdot) \mathrm{d} \mu_{t}-\int_{\mathbb{R}^{d}} \mathrm{u}(s, \cdot) \mathrm{d} \mu_{s}+C
$$

for the constant $C=\left(\|\alpha\|_{\mathcal{X}^{q}(Q)}+\left\|\gamma_{H}^{-}\right\|_{\mathcal{X}^{q}\left(\mathbb{R}^{d}\right)}\right)\|m\|_{L^{p} \cap L_{\kappa}^{1}(Q)}+c_{H} \int_{Q}|\boldsymbol{v}|^{2} \mathrm{~d} \tilde{\mu}$.

Finaly, using the fact that $Z, \zeta$ are bounded and continuous, we can pass to the limit in (5.44) obtaining the weak formulation of (5.39) supplemented with boundary terms:

$$
\begin{aligned}
-\int_{Q} Z(u) m\left(\partial_{t} \varphi+D \varphi \cdot \boldsymbol{v}\right) \mathrm{d} x \mathrm{~d} t & +\int_{Q} \zeta(u)(\alpha-H(x, D u)-D u \cdot \boldsymbol{v}) m \varphi \mathrm{d} x \mathrm{~d} t \\
& \geq \int_{\mathbb{R}^{d}} Z\left(u^{+}(0, x)\right) \varphi(0, x) \mathrm{d} \mu_{0}(x)-\int_{\mathbb{R}^{d}} Z\left(u^{-}(1, x)\right) \varphi(1, x) \mathrm{d} \mu_{1}(x),
\end{aligned}
$$

for every nonnegative $\varphi \in C_{c}^{1}(\bar{Q})$.

Step 2. Let us now deal with the case of a general subsolution $u$. If we define $u_{k}:=-k \vee u \wedge k$, from Corollary 5.4 we know that $u_{k}$ is a bounded weak subsolution to

$$
-\partial_{t} u_{k}+H\left(x, D u_{k}\right) \leq \alpha_{k}
$$

where $\alpha_{k} \rightarrow \alpha$ weakly* in $\mathcal{X}^{q}(Q)$ and pointwise a.e., with the uniform domination $\left|\alpha_{k}\right| \leq|\alpha|+\gamma_{H}^{-}$. In particular (5.47), (5.48) and (5.49) hold with $u_{k}, \alpha_{k}$ in place of $u, \alpha$ respectively.

(5.39) and (5.40) can be directly obtained by choosing $k$ sufficiently big, so that $\operatorname{supp}(\zeta) \subset(-k, k)$, since in this case

$$
Z(u)=Z\left(u_{k}\right), \quad \zeta(u)=\zeta\left(u_{k}\right), \quad \zeta(u) \alpha=\zeta\left(u_{k}\right) \alpha_{k}
$$

In order to prove (5.38) and (5.41), we recall that $\mathrm{u}(t, \cdot)$ belongs to $\mathcal{X}^{q}\left(\mathbb{R}^{d}\right)$ for every $t \in(0,1)$ (with a uniform estimate in any compact subset $[a, b])$. If we choose $s, t \in D_{p}[\mu]$ we thus have $\mathrm{u}(t, \cdot) \in L_{\mu_{t}}^{1}\left(\mathbb{R}^{d}\right)$, $\mathrm{u}(s, \cdot) \in L_{\mu_{s}}^{1}\left(\mathbb{R}^{d}\right)$, so that the right hand side of (5.48) is uniformly bounded and (5.38) follows from Fatou's lemma.

(5.41) is a consequence of a similar limit starting from

$$
\int_{\mathbb{R}^{d}} \mathbf{u}_{k}(s, x) \mathrm{d} \mu_{s}(x)-\int_{\mathbb{R}^{d}} \mathbf{u}_{k}(t, x) \mathrm{d} \mu_{t}(x) \leq \int_{s}^{t} \int_{\mathbb{R}^{d}}\left(\alpha_{k}-H\left(x, D u_{k}\right)-D u_{k} \cdot \boldsymbol{v}\right) m \mathrm{~d} x \mathrm{~d} r
$$

and applying Lebesgue Dominated Convergence Theorem (and Fatou's lemma in the right-hand side if $t=1$ or $s=0)$.

For our next purposes, we deduce the following consequence of inequality (5.41), which is a natural extension of (5.16). The proof follows the same argument of Corollary 5.9, starting from (5.41).

Corollary 5.13 Let $(u, \alpha) \in \mathrm{HJ}_{q}(Q, H)$ and $H$ satisfying (2.13). Let $\mu_{i}=m_{i} \mathscr{L}^{d} \in \mathcal{P}_{2, p}^{r}\left(\mathbb{R}^{d}\right)$. Then we have

$$
\int_{\mathbb{R}^{d}} u_{0}^{+} \mathrm{d} \mu_{0}-\int_{\mathbb{R}^{d}} u_{1}^{-} \mathrm{d} \mu_{1} \leq \frac{c_{H}}{2} W_{2}^{2}\left(\mu_{0}, \mu_{1}\right)+\|\beta\|_{\mathcal{X}^{q}(Q)} \max \left(\left\|m_{0}\right\|_{L^{p} \cap L_{\kappa}^{1}\left(\mathbb{R}^{d}\right)},\left\|m_{1}\right\|_{L^{p} \cap L_{\kappa}^{1}\left(\mathbb{R}^{d}\right)}\right),
$$

where $\beta:=\alpha+\gamma_{H}^{-}$. 


\subsection{Contact-defect measures associated to weak subsolutions}

In this last section we rewrite (5.41), (5.40), and (5.39) in a more expressive way.

Let us fix a pair $(u, \alpha) \in \operatorname{HJ}_{q}(Q, H)$ and a solution $(m, \boldsymbol{v}) \in \mathrm{CE}_{2, p}\left(Q ; \mu_{0}, \mu_{1}\right)$ with $\mu_{i} \ll \mathscr{L}^{d}, i=0,1$. First of all, for every $\zeta \in C_{c}^{0}(\mathbb{R})$ with $Z(r):=\int_{0}^{r} \zeta(s) \mathrm{d} s$ we consider the linear functional $T_{\zeta} \in \mathscr{D}^{\prime}\left(\mathbb{R}^{d+1}\right)$

$$
\begin{aligned}
T_{\zeta}(\varphi):=-\int_{Q} Z(u) m & \left(\partial_{t} \varphi+D \varphi \cdot \boldsymbol{v}\right) \mathrm{d} x \mathrm{~d} t+\int_{Q} \zeta(u)(\alpha-H(x, D u)-D u \cdot \boldsymbol{v}) m \varphi \mathrm{d} x \mathrm{~d} t \\
& -\left(\int_{\mathbb{R}^{d}} Z\left(u^{+}(0, x)\right) \varphi(0, x) \mathrm{d} \mu_{0}(x)-\int_{\mathbb{R}^{d}} Z\left(u^{-}(1, x)\right) \varphi(1, x) \mathrm{d} \mu_{1}(x)\right)
\end{aligned}
$$

for every $\varphi \in C_{c}^{\infty}\left(\mathbb{R}^{d+1}\right)$. Since $\varphi, \zeta \geq 0$ yield $T_{\zeta}(\varphi) \geq 0$ and $\operatorname{supp}(\varphi) \subset \mathbb{R}^{d+1} \backslash Q \Rightarrow T_{\zeta}(\varphi)=0$, we know that there exists a unique nonnegative Radon measure $\vartheta_{\zeta}$ such that

$$
T_{\zeta}(\varphi)=\int_{\mathbb{R} \times \mathbb{R}^{d}} \varphi(t, x) \mathrm{d} \vartheta_{\zeta}(t, x) \quad \text { for every } \varphi \in C_{c}^{\infty}\left(\mathbb{R}^{d+1}\right), \quad \operatorname{supp}\left(\vartheta_{\zeta}\right)=\bar{Q}
$$

In this way, $T_{\zeta}$ can be extended to a linear positive functional on $C_{c}^{0}\left(\mathbb{R}^{d+1}\right)$. In particular, choosing $\varphi$ with compact support in $Q$

$$
\partial_{t}(Z(u) m)+\nabla \cdot(Z(u) m \boldsymbol{v})+\zeta(u)(\alpha-H(x, D u)-D u \cdot \boldsymbol{v}) m=\vartheta_{\zeta} \quad \text { in } \mathscr{D}^{\prime}(Q) .
$$

We want now to associate a nonnegative Radon measure $\vartheta$ on $\mathbb{R} \times \mathbb{R}^{d+1}$ to $T$ so that

$$
T_{\zeta}(\varphi)=\int_{\mathbb{R} \times \bar{Q}} \zeta(r) \varphi(t, x) \mathrm{d} \vartheta(r, t, x) \quad \text { for every } \zeta \in C_{c}^{0}(\mathbb{R}), \varphi \in C_{c}^{0}\left(\mathbb{R}^{d+1}\right) .
$$

Proposition 5.14 For every $(u, \alpha) \in \operatorname{HJ}_{q}(Q, H)$ and $(m, v) \in \mathrm{CE}_{2, p}\left(Q ; \mu_{0}, \mu_{1}\right)$ with $\mu_{i} \ll \mathscr{L}^{d}$, there exists a unique positive contact-defect Radon measure $\vartheta$ on $\mathbb{R} \times \mathbb{R}^{d+1}$ satisfying (5.55). Moreover, $\vartheta$ is supported on $\mathbb{R} \times \bar{Q}$.

Proof. The functional $(\zeta, \varphi) \mapsto T_{\zeta}(\varphi)$ is bilinear on $C_{c}^{0}(\mathbb{R}) \times C_{c}^{0}\left(\mathbb{R}^{d+1}\right)$ and it is positive on positive functions, so the representation formula (5.55) follows by the extension of Riesz representation Theorem to positive bilinear functionals, see Theorem A.3 in the Appendix.

Let us now write the distributional definition of $T_{\zeta}$ in a slightly different way. We introduce the nonnegative functions $Y_{H}, Y_{F}$ related to Fenchel duality between the pairs $H, L$ and $F, F^{*}$ :

$$
\begin{aligned}
Y_{H}(x, \boldsymbol{p}, \boldsymbol{v}) & :=H(x, \boldsymbol{p})+\boldsymbol{p} \cdot \boldsymbol{v}+L(x, \boldsymbol{v}), \quad x, \boldsymbol{p}, \boldsymbol{v} \in \mathbb{R}^{d} \\
Y_{F}(x, m, \alpha) & :=F(x, m)-\alpha m+F^{*}(x, \alpha), \quad x \in \mathbb{R}^{d}, \alpha \in \mathbb{R}, m \geq 0 .
\end{aligned}
$$

Corollary 5.15 Let $(u, \alpha) \in \mathrm{HJ}_{q}(Q, H)$ and let $(m, \boldsymbol{v}) \in \mathrm{CE}_{2, p}\left(Q ; \mu_{0}, \mu_{1}\right)$, and let us consider the measurable functions

$$
\mathrm{Y}_{H}:=Y_{H}(\cdot, D u, \boldsymbol{v}), \quad \mathrm{Y}_{F}:=Y_{F}(\cdot, m, \alpha), \quad \mathrm{L}:=L(\cdot, \boldsymbol{v}), \quad \mathrm{F}:=F(\cdot, m), \quad \mathrm{F}^{*}:=F^{*}(\cdot, \alpha) .
$$

For every $\zeta \in C_{c}^{0}(\mathbb{R})$ and $Z \in C_{b}^{1}(\mathbb{R})$ with $Z^{\prime}=\zeta$ we have

$$
\zeta(u)\left(\mathrm{Y}_{H} m+\mathrm{Y}_{F}\right)+\vartheta_{\zeta}=\zeta(u)\left(\mathrm{L} m+\mathrm{F}+\mathrm{F}^{*}\right)+\partial_{t}(Z(u) m)+\nabla \cdot(Z(u) m \boldsymbol{v})
$$

in the sense of distributions of $\mathscr{D}^{\prime}(Q)$, and also in duality with functions $\varphi \in C_{c}^{1}(\bar{Q})$ if $\mu_{0}, \mu_{1} \ll \mathscr{L}^{d}$ :

$$
\begin{aligned}
& \int_{Q} \zeta(u)\left(\mathrm{Y}_{H} m+\mathrm{Y}_{F}\right) \varphi \mathrm{d} x \mathrm{~d} t+\int_{\mathbb{R} \times \mathbb{R}^{d+1}} \zeta(u) \varphi(t, x) \mathrm{d} \vartheta(u, t, x) \\
& =\int_{Q} \zeta(u)(\mathrm{L} m+\mathrm{F}) \varphi \mathrm{d} x \mathrm{~d} t-\int_{Q} Z(u) m\left(\partial_{t} \varphi+D \varphi \cdot \boldsymbol{v}\right) \mathrm{d} x \mathrm{~d} t \\
& \quad-\left(\int_{\mathbb{R}^{d}} Z\left(u^{+}(0, x)\right) \varphi(0, x) \mathrm{d} \mu_{0}(x)-\int_{\mathbb{R}^{d}} Z\left(u^{-}(1, x)\right) \varphi(1, x) \mathrm{d} \mu_{1}(x)-\int_{Q} \zeta(u) \mathrm{F}^{*} \varphi \mathrm{d} x \mathrm{~d} t\right) .
\end{aligned}
$$


Proof. The proof is a simple manipulation of (5.54) and (5.52). Let us check e.g. (5.59), starting directly from (5.54):

$$
\begin{aligned}
\zeta(u) & \left(\mathrm{L} m+\mathrm{F}+\mathrm{F}^{*}\right)+\partial_{t}(Z(u) m)+\nabla \cdot(Z(u) m \boldsymbol{v}) \\
& =\zeta(u)\left(\mathrm{L} m+\mathrm{F}+\mathrm{F}^{*}\right)-\zeta(u)(\alpha-\mathrm{H}-D u \cdot \boldsymbol{v}) m+\vartheta_{\zeta} \\
& =\zeta(u)(\mathrm{L} m+\mathrm{H}+D u \cdot \boldsymbol{v})+\zeta(u)\left(\mathrm{F}-\alpha m+\mathrm{F}^{*}\right)+\vartheta_{\zeta}=\zeta(u)\left(\mathrm{Y}_{H} m+\mathrm{Y}_{F}\right)+\vartheta_{\zeta} .
\end{aligned}
$$

We conclude this section by deriving an important formula concerning the total mass of the measures $\vartheta_{\zeta}$ and $\vartheta$.

Theorem 5.16 Let $(u, \alpha) \in \operatorname{HJ}_{q}(Q, H)$ and let $(m, v) \in \mathrm{CE}_{2, p}\left(Q ; \mu_{0}, \mu_{1}\right)$ be a distributional solution to the continuity equation according to Definition [3.3, and let us keep the notation of (5.56), (5.57), (5.58). If $\mu_{0}, \mu_{1} \ll \mathscr{L}^{d}$, for every nonnegative $\zeta \in C_{c}^{0}(\mathbb{R}) \vartheta_{\zeta}$ has finite total mass and for every $\eta \in C_{c}^{1}(\mathbb{R}), \zeta \in C_{c}^{0}(\mathbb{R})$ we have

$$
\begin{aligned}
& \int_{Q} \zeta(u)\left(\mathrm{Y}_{H} m+\mathrm{Y}_{F}\right) \eta(t) \mathrm{d} x \mathrm{~d} t+\int_{\bar{Q}} \eta \mathrm{d} \vartheta_{\zeta}+\int_{Q} Z(u) m \eta^{\prime}(t) \mathrm{d} x \mathrm{~d} t \\
& \quad=\int_{Q} \zeta(u)(\mathrm{L} m+\mathrm{F}) \eta(t) \mathrm{d} x \mathrm{~d} t-\left(\int_{\mathbb{R}^{d}} Z\left(u_{0}^{+}\right) \eta_{0} \mathrm{~d} \mu_{0}-\int_{\mathbb{R}^{d}} Z\left(u_{1}^{-}\right) \eta_{1} \mathrm{~d} \mu_{1}-\int_{Q} \eta(t) \zeta(u) \mathrm{F}^{*} \mathrm{~d} x \mathrm{~d} t\right) \\
& \int_{Q} \zeta(u)\left(\mathrm{Y}_{H} m+\mathrm{Y}_{F}\right) \mathrm{d} x \mathrm{~d} t+\vartheta_{\zeta}(\bar{Q}) \\
& \quad=\int_{Q} \zeta(u)(\mathrm{L} m+\mathrm{F}) \mathrm{d} x \mathrm{~d} t-\left(\int_{\mathbb{R}^{d}} Z\left(u_{0}^{+}\right) \mathrm{d} \mu_{0}-\int_{\mathbb{R}^{d}} Z\left(u_{1}^{-}\right) \mathrm{d} \mu_{1}-\int_{Q} \zeta(u) \mathrm{F}^{*} \mathrm{~d} x \mathrm{~d} t\right) .
\end{aligned}
$$

Moreover, for every $0<s<t<1$ we have $\vartheta\left(\mathbb{R} \times \overline{Q_{s, t}}\right)<\infty$ and there exists an at most countable set $J \subset(0,1)$ such that for every $s, t \in D_{p}[\mu] \backslash J$ we have $\vartheta\left(\mathbb{R} \times\{s\} \times \mathbb{R}^{d}\right)=\vartheta\left(\mathbb{R} \times\{t\} \times \mathbb{R}^{d}\right)=0$ and

$$
\int_{Q_{s, t}}\left(\mathrm{Y}_{H} m+\mathrm{Y}_{F}\right) \mathrm{d} \lambda+\vartheta\left(\mathbb{R} \times \overline{Q_{s, t}}\right)=\int_{Q_{s, t}}(\mathrm{~L} m+\mathrm{F}) \mathrm{d} x \mathrm{~d} t-\left(\int_{\mathbb{R}^{d}} \mathrm{u}_{s} \mathrm{~d} \mu_{s}-\int_{\mathbb{R}^{d}} \mathrm{u}_{t} \mathrm{~d} \mu_{t}-\int_{Q_{s, t}} \mathrm{~F}^{*} \mathrm{~d} x \mathrm{~d} t\right) .
$$

(5.63) also holds at $s=0$ (resp. $t=1)$ if $\int_{\mathbb{R}}\left(u_{0}^{+} \wedge 0\right) \mathrm{d} \mu_{0}>-\infty$ (resp. $\left.\int_{\mathbb{R}}\left(u_{1}^{-} \vee 0\right) \mathrm{d} \mu_{1}<+\infty\right)$. If both these integrals are finite then $\vartheta$ has finite total mass and we have

$$
\int_{Q}\left(\mathrm{Y}_{H} m+\mathrm{Y}_{F}\right) \mathrm{d} x \mathrm{~d} t+\vartheta(\mathbb{R} \times \bar{Q})=\int_{Q}(\mathrm{~L} m+\mathrm{F}) \mathrm{d} x \mathrm{~d} t-\left(\int_{\mathbb{R}^{d}} u_{0}^{+} \mathrm{d} \mu_{0}-\int_{\mathbb{R}^{d}} u_{1}^{-} \mathrm{d} \mu_{1}-\int_{Q} \mathrm{~F}^{*} \mathrm{~d} x \mathrm{~d} t\right) .
$$

Proof. Let us fix a nonnegative radial test function $\xi: \mathbb{R}^{d} \rightarrow[0,1]$ of class $C^{1}$ such that

$$
\xi(x) \equiv 1 \text { if }|x| \leq 1, \quad \xi(x) \equiv 0 \text { if }|x| \geq 2 ; \quad \xi_{k}(x):=\xi\left(2^{-k} x\right) .
$$

Notice that $\xi_{k}$ is increasing w.r.t. $k$; we also set $\varphi_{k}(t, x):=\eta(t) \xi_{k}(x)$ and we write (5.60) for a fixed nonnegative $\zeta$ and $\varphi:=\varphi_{k}$. We notice that

$$
\begin{gathered}
\partial_{t} \varphi_{k} \equiv \eta^{\prime} \xi_{k} \text { on } Q, \\
\int_{Q} Z(u) m \eta\left|D \xi_{k} \cdot \boldsymbol{v}\right| \mathrm{d} t \mathrm{~d} x \leq 2^{-k}\left(\sup _{\mathbb{R}^{d}}|D \xi|\right)\left(\sup _{\mathbb{R}} Z\right)\left(\sup _{\mathbb{R}} \eta\right) \int_{Q}|\boldsymbol{v}|^{2} m \mathrm{~d} x \mathrm{~d} t \rightarrow 0 \quad \text { as } k \rightarrow \infty ;
\end{gathered}
$$

since $\varphi_{k} \uparrow 1$, applying Lebesgue Dominated Convergence Theorem we easily get

$$
\begin{aligned}
\lim _{k \rightarrow \infty} \int_{Q} \zeta(u)\left(\mathrm{Y}_{H} m+\mathrm{Y}_{F}\right) \varphi_{k} \mathrm{~d} x \mathrm{~d} t & =\int_{Q} \zeta(u)\left(\mathrm{Y}_{H} m+\mathrm{Y}_{F}\right) \eta \mathrm{d} x \mathrm{~d} t \\
\lim _{k \rightarrow \infty} \int_{Q} Z(u) m \eta^{\prime}(t) \varphi_{k} \mathrm{~d} x \mathrm{~d} t & =\int_{Q} Z(u) m \eta^{\prime}(t) \mathrm{d} x \mathrm{~d} t
\end{aligned}
$$

$\lim _{k \rightarrow \infty} \int_{Q} \zeta(u)(\mathrm{L} m+\mathrm{F}) \varphi_{k} \mathrm{~d} x \mathrm{~d} t=\int_{Q} \zeta(u)(\mathrm{L} m+\mathrm{F}) \eta \mathrm{d} x \mathrm{~d} t, \quad \lim _{k \rightarrow \infty} \int_{Q} \zeta(u) \mathrm{F}^{*} \varphi_{k} \mathrm{~d} x \mathrm{~d} t=\int_{Q} \zeta(u) \eta \mathrm{F}^{*} \mathrm{~d} x \mathrm{~d} t$

$$
\lim _{k \rightarrow \infty} \int_{\mathbb{R}^{d}} Z\left(u_{0}^{+}\right) \varphi_{k}(0, \cdot) \mathrm{d} \mu_{0}=\int_{\mathbb{R}^{d}} Z\left(u_{0}^{+}\right) \eta_{0} \mathrm{~d} \mu_{0}, \quad \lim _{k \rightarrow \infty} \int_{\mathbb{R}^{d}} Z\left(u_{1}^{-}\right) \varphi_{k}(1, \cdot) \mathrm{d} \mu_{1}=\int_{\mathbb{R}^{d}} Z\left(u_{1}^{-}\right) \eta_{1} \mathrm{~d} \mu_{1} .
$$


Eventually, choosing $\zeta \geq 0$ and $\eta \geq 0$, Beppo Levi monotone convergence theorem yields

$$
\lim _{k \rightarrow \infty} \int_{\mathbb{R} \times \mathbb{R}^{d+1}} \zeta(u) \varphi_{k}(t, x) \mathrm{d} \vartheta(u, t, x)=\int_{\mathbb{R} \times \mathbb{R}^{d+1}} \eta(t) \zeta(u) \mathrm{d} \vartheta(u, t, x)
$$

so that

$$
\begin{aligned}
\int_{Q} \zeta(u)\left(\mathrm{Y}_{H} m+\mathrm{Y}_{F}\right) \eta(t) \mathrm{d} x \mathrm{~d} t+\int_{\mathbb{R} \times \mathbb{R}^{d+1}} \eta(t) \zeta(u) \mathrm{d} \vartheta(u, t, x)+\int_{Q} Z(u) \eta^{\prime}(t) m \mathrm{~d} x \mathrm{~d} t \\
\quad=\int_{Q} \zeta(u)(\mathrm{L} m+\mathrm{F}) \eta(t) \mathrm{d} x \mathrm{~d} t-\left(\int_{\mathbb{R}^{d}} Z\left(u_{0}^{+}\right) \mathrm{d} \mu_{0}-\int_{\mathbb{R}^{d}} Z\left(u_{1}^{-}\right) \mathrm{d} \mu_{1}-\int_{Q} \zeta(u) \eta(t) \mathrm{F}^{*} \mathrm{~d} x \mathrm{~d} t\right) .
\end{aligned}
$$

Writing the above identity for the positive and the negative part of $\zeta$, we obtain that it holds for every $\zeta \in C_{c}^{0}(\mathbb{R})$, together with its $\eta$-localized version (5.61).

We can now write (5.65) by choosing $\eta \geq 0$ with compact support in $(0,1)$ and $\eta \equiv 1$ on the interval $[s, t]$ and choosing an increasing sequence of nonnegative functions $\zeta_{k} \in C_{c}^{0}(\mathbb{R})$ converging to 1 , so that $\left(Z_{k}(u) \vee 0\right):=\int_{0}^{u \vee 0} \zeta_{k}(v) \mathrm{d} v$ is monotonically increasing to $u \vee 0$ and $\left(Z_{k}(u) \wedge 0\right):=-\int_{u \wedge 0}^{0} \zeta_{k}(v) \mathrm{d} v$ is monotonically decreasing to $u \wedge 0$. The application of Beppo Levi's and Lebesgue dominated convergence theorem yields

$$
\begin{gathered}
\int_{Q}\left(\mathrm{Y}_{H} m+\mathrm{Y}_{F}\right) \eta(t) \mathrm{d} x \mathrm{~d} t+\int_{\mathbb{R}_{\mathbb{R}^{d+1}}} \eta(t) \mathrm{d} \vartheta(u, t, x)+\int_{Q} u \eta^{\prime}(t) m \mathrm{~d} x \mathrm{~d} t \\
=\int_{Q}(\mathrm{~L} m+\mathrm{F}) \eta(t) \mathrm{d} x \mathrm{~d} t+\int_{Q} \zeta(u) \eta(t) \mathrm{F}^{*} \mathrm{~d} x \mathrm{~d} t
\end{gathered}
$$

which in particular shows that $\vartheta\left(\mathbb{R} \times Q_{s, t}\right)<\infty$ for every $0<s<t<1$. The set $J_{1}:=\{s \in(0,1)$ : $\left.\vartheta\left(\mathbb{R} \times\{s\} \times \mathbb{R}^{d}\right)>0\right\}$ is therefore at most countable and $D_{p}[\mu] \backslash J_{1}$ has full measure in $(0,1)$. On the other hand, (5.41) shows that the map

$$
t \mapsto \int_{\mathbb{R}^{d}} \mathrm{u}_{t} \mathrm{~d} \mu_{t}+\int_{1 / 2}^{t} \int_{\mathbb{R}^{d}}(\alpha-H(x, D u)-D u \cdot \boldsymbol{v}) m \mathrm{~d} x \mathrm{~d} r, \quad t \in D_{p}[\mu]
$$

is finite and increasing in $D_{p}[\mu]$, so that it has an at most countable jump set $J_{2}$. Setting $J:=J_{1} \cup J_{2}$ and choosing a decreasing sequence $\eta_{h}$ such that $\eta_{h} \equiv 1$ on $[s, t], \eta_{h} \in[0,1]$, and $\eta_{h} \downarrow 0$ in $\mathbb{R} \backslash[s, t]$, (5.66) yields (5.63) at every $s, t \in D_{p}[\mu] \backslash J$.

A similar argument holds for $s=0$ (resp. $t=1)$ by assuming $\int_{\mathbb{R}}\left(u_{0}^{+} \wedge 0\right) \mathrm{d} \mu_{0}>-\infty\left(\right.$ resp. $\int_{\mathbb{R}}\left(u_{1}^{-} \vee 0\right) \mathrm{d} \mu_{1}<$ $+\infty)$ and choosing a function $\eta$ identically 1 in the interval $[-1, t](\operatorname{resp} .[s, 2])$.

We deduce from the previous Theorem a precise characterization of the optimality conditions. In order to express in a convenient way the trace conditions, we will use approximate limits: recall that for a measurable map $g: A \rightarrow \mathbb{R}$ (a.e.) defined in a measurable subset $A \subset \mathbb{R}$ and a point $t_{0} \in \mathbb{R}$ of positive density for $A$ we have

$$
\begin{aligned}
& \underset{t \rightarrow t_{0}}{\operatorname{ap}-\liminf } g(t):=\sup \left\{\ell \in \mathbb{R}: \lim _{r \downarrow 0} r^{-1} \mathscr{L}^{1}\left(\left\{t \in A:\left|t-t_{0}\right|<r, g(t)<\ell\right\}\right)=0\right\}, \\
& \underset{t \rightarrow t_{0}}{\operatorname{ap}-\operatorname{limup}} g(t):=\inf \left\{\ell \in \mathbb{R}: \lim _{r \downarrow 0} r^{-1} \mathscr{L}^{1}\left(\left\{t \in A:\left|t-t_{0}\right|<r, g(t)>\ell\right\}\right)=0\right\} \text {, }
\end{aligned}
$$

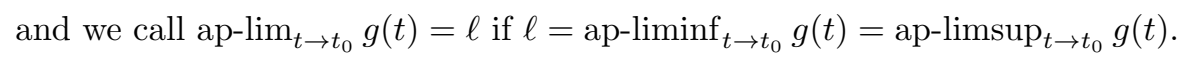

Corollary 5.17 Let $(u, \alpha) \in \operatorname{HJ}_{q}(Q, H)$ and let $(m, \boldsymbol{v}) \in \mathrm{CE}_{2, p}\left(Q ; \mu_{0}, \mu_{1}\right)$ with $\mu_{0}, \mu_{1} \ll \mathscr{L}^{d}$ and $\int_{\mathbb{R}^{d}}\left(u_{0}^{+} \vee\right.$ 0) $\mathrm{d} \mu_{0}<+\infty, \int_{\mathbb{R}^{d}}\left(u_{1}^{-} \wedge 0\right) \mathrm{d} \mu_{1}>-\infty$. Let us set

$$
\begin{aligned}
\mathcal{A}(u, \alpha) & :=\int_{\mathbb{R}^{d}} u_{0}^{+} \mathrm{d} \mu_{0}-\int_{\mathbb{R}^{d}} u_{1}^{-} \mathrm{d} \mu_{1}-\int_{Q} F^{*}(x, \alpha(t, x)) \mathrm{d} x \mathrm{~d} t \in[-\infty,+\infty) \\
\mathcal{B}(m, \boldsymbol{v}) & :=\int_{Q}(L(x, \boldsymbol{v}(t, x)) m(t, x)+F(x, m(t, x))) \mathrm{d} x \mathrm{~d} t,
\end{aligned}
$$

Then

$$
\mathcal{A}(u, \alpha) \leq \mathcal{B}(m, \boldsymbol{v})
$$

and the equality holds in (5.70) if and only if $u_{i} \in L^{1}\left(\mathbb{R}^{d}, \mu_{i}\right)$ and

$$
\begin{aligned}
Y_{H}(x, D u, \boldsymbol{v})=0 & \text { for } \tilde{\mu} \text {-a.e. }(t, x) \in Q, \\
Y_{F}(x, \alpha, m)=0 & \text { for } \mathscr{L}^{d+1} \text {-a.e. }(t, x) \in Q, \\
\vartheta(\mathbb{R} \times \bar{Q})=0 . &
\end{aligned}
$$


Moreover, $\vartheta(\mathbb{R} \times \bar{Q})=0$ if and only if for every nonnegative $\zeta \in C_{c}^{0}(\mathbb{R})$

$$
\partial_{t}(Z(u) m)+\nabla \cdot(Z(u) m \boldsymbol{v})+\zeta(u)(\alpha-H(x, D u)-D u \cdot \boldsymbol{v}) m=0 \quad \text { in } \mathscr{D}^{\prime}(Q),
$$

and the traces $u_{0}^{+}$and $u_{1}^{-}$are taken in the following weak sense

$$
\underset{t \downarrow 0}{\operatorname{ap}-\lim } \int_{\mathbb{R}^{d}} u(t, x) \mathrm{d} \mu_{t}=\int_{\mathbb{R}^{d}} u_{0}^{+} \mathrm{d} \mu_{0}, \quad \underset{t \uparrow 1}{\operatorname{ap}-\lim } \int_{\mathbb{R}^{d}} u(t, x) \mathrm{d} \mu_{t}=\int_{\mathbb{R}^{d}} u_{1}^{-} \mathrm{d} \mu_{1} .
$$

Proof. (5.70) clearly follows from (5.63) since $\vartheta$ is a positive measure (notice that if the integral of the negative part of $u_{0}^{+}$w.r.t. $\mu_{0}$ or the integral of the positive part of $u_{1}^{-}$w.r.t. $\mu_{1}$ are not finite, $\mathcal{A}(u, \alpha)=-\infty$ and (5.70) is trivially satisfied). The optimality conditions (5.71) are also an immediate consequence of (5.63), since $Y_{H}$ and $Y_{F}$ are nonnegative.

Let us check the last statement. If $\vartheta(\mathbb{R} \times \bar{Q})=0$ then (5.72) is an immediate consequence of (5.54). (5.63) shows that the map $t \mapsto \int_{\mathbb{R}^{d}} \mathrm{u}(t, x) \mathrm{d} \mu_{t}(x), t \in D_{p}[\mu]$, is the restriction to $D_{p}[\mu]$ of an absolutely continuous map with limits $\int_{\mathbb{R}^{d}} u_{0}^{+} \mathrm{d} \mu_{0}$ (resp. $\left.\int_{\mathbb{R}^{d}} u_{1}^{-} \mathrm{d} \mu_{1}\right)$ as $t \downarrow 0$ (resp. as $t \uparrow 1$ ), so that (5.73) holds.

Conversely, (5.72) shows that $\vartheta(\mathbb{R} \times Q)=0$. (5.63) then shows that there exists an at most countable set $J$ such that

$$
\vartheta\left(\mathbb{R} \times\{0\} \times \mathbb{R}^{d}\right)=I(t)+\int_{\mathbb{R}^{d}} \mathrm{u}_{t} \mathrm{~d} \mu_{t}-\int_{\mathbb{R}^{d}} u_{0}^{+} \mathrm{d} \mu_{0} \quad \text { for every } t \in D_{p}[\mu] \backslash J,
$$

where

$$
I(t):=\int_{Q_{0, t}}\left(\left(\mathrm{~L}-\mathrm{Y}_{H}\right) m+\left(\mathrm{F}-\mathrm{Y}_{F}-\mathrm{F}^{*}\right)\right) \mathrm{d} \lambda
$$

Since $\lim _{t \downarrow 0} I(t)=0$ (5.73) yields $\vartheta\left(\mathbb{R} \times\{0\} \times \mathbb{R}^{d}\right)=0$. A similar argument can be used to show $\vartheta(\mathbb{R} \times\{1\} \times$ $\left.\mathbb{R}^{d}\right)=0$

Notice that it always holds

$$
\underset{t \downarrow 0}{\operatorname{ap}-\liminf } \int_{\mathbb{R}^{d}} u(t, x) \mathrm{d} \mu_{t} \geq \int_{\mathbb{R}^{d}} u_{0}^{+} \mathrm{d} \mu_{0}, \quad \operatorname{ap-limsup} \int_{t \uparrow 1} u(t, x) \mathrm{d} \mu_{t} \leq \int_{\mathbb{R}^{d}} u_{1}^{-} \mathrm{d} \mu_{1},
$$

so that (5.73) is in fact equivalent to the property

$$
\underset{t \downarrow 0}{\operatorname{ap}-\limsup } \int_{\mathbb{R}^{d}} u(t, x) \mathrm{d} \mu_{t} \leq \int_{\mathbb{R}^{d}} u_{0}^{+} \mathrm{d} \mu_{0}, \quad \underset{t \uparrow 1}{\operatorname{ap}-\operatorname{limininf}} \int_{\mathbb{R}^{d}} u(t, x) \mathrm{d} \mu_{t} \geq \int_{\mathbb{R}^{d}} u_{1}^{-} \mathrm{d} \mu_{1} .
$$

\section{A Variational approach to MFPP}

It is known (see [20]) that solutions to MFG systems can be, at least formally, characterized as minimizers of two optimal control problems. Here we adopt the same strategy in the context of MFPPs. Following the standard approach of the dynamical formulation of Optimal Transport problems, we first study a primal formulation, consisting in the minimization of a convex action-entropy functional among all the solutions $(m, \boldsymbol{v}) \in \mathrm{CE}_{2, p}\left(Q ; \mu_{0}, \mu_{1}\right)$ to the continuity equation connecting two given measures $\mu_{i} \in \mathcal{P}_{2}\left(\mathbb{R}^{d}\right)$.

The dual problem naturally arises as a supremum of a concave cost functional, defined on subsolutions to the Hamilton-Jacobi equation.

As usual, in all this section we will refer to the main structural conditions stated in 2.1

\subsection{The variational structure of the primal problem}

We state the primal problem for an arbitrary pair of measures $\mu_{i} \in \mathcal{P}_{2}\left(\mathbb{R}^{d}\right)$; at this stage, the minimal assumption requires that $\mathrm{CE}_{2, p}\left(Q ; \mu_{0}, \mu_{1}\right)$ is not empty (or, equivalently, that $\mu_{i} \in \mathcal{P}_{2, p}\left(\mathbb{R}^{d}\right)$ ) and we do not need to add any other a priori regularity on $\mu_{0}, \mu_{1}$.

Problem 6.1 Let $\mu_{i} \in \mathcal{P}_{2}\left(\mathbb{R}^{d}\right)$ be given. We look for the minimizer of the functional

$$
\mathcal{B}(m, \boldsymbol{v}):=\int_{0}^{1} \int_{\mathbb{R}^{d}}(L(x, \boldsymbol{v}(t, x)) m(t, x)+F(x, m(t, x))) \mathrm{d} x \mathrm{~d} t,
$$

among all solutions $(m, \boldsymbol{v}) \in \mathrm{CE}_{2, p}\left(Q ; \mu_{0}, \mu_{1}\right)$ of the continuity equation connecting $\mu_{0}$ to $\mu_{1}$. 
In order to prove the existence of a solution to Problem 6.1. we first show that a control of $m$ in $L_{1+|x|}^{1}(Q)$ yields uniform bounds of the sublevels of $\mathcal{B}$. Notice that at this first stage we are not assuming that $(m, \boldsymbol{v})$ solves the continuity equation nor that $m$ has integral 1.

Lemma 6.2 (A priori estimates I) Let $m \in L_{\kappa}^{1}(Q)$ be nonnegative and let $\boldsymbol{v}: Q \rightarrow \mathbb{R}^{d}$ be a Borel vector field satisfying

$$
\begin{array}{r}
\int_{Q}(1+|x|) m(t, x) \mathrm{d} x \mathrm{~d} t \leq M \\
\mathcal{B}(m, \boldsymbol{v}) \leq B
\end{array}
$$

for suitable constants $M, B>0$. Then $\boldsymbol{w}:=m \boldsymbol{v} \in L^{2 p /(p+1)}(Q)$ and there exists a constant $E>0$ only depending on $B, M$, and on the structural constants

$$
c_{H}, c_{H}^{ \pm}, c_{f}, C_{f}:=\left\|\gamma_{f}\right\|_{L^{q}\left(\mathbb{R}^{d}\right)}
$$

such that

$$
\int_{Q}\left(|\boldsymbol{v}|^{2} m+m^{p}\right) \mathrm{d} x \mathrm{~d} t \leq E, \quad \int_{Q}|\boldsymbol{w}|^{\frac{2 p}{p+1}} \mathrm{~d} x \mathrm{~d} t \leq E .
$$

Proof. Let us first observe that (2.15) and the elementary inequalities

$$
\gamma_{f} m \leq \frac{1}{p\left(2 c_{f}\right)^{p}} m^{p}+\frac{\left(2 c_{f}\right)^{q}}{q} \gamma_{f}^{q}
$$

yield

$$
\int_{Q} F(x, m) \mathrm{d} x \mathrm{~d} t \geq \frac{1}{2 p c_{f}^{p}} \int_{Q} m^{p} \mathrm{~d} x \mathrm{~d} t-C_{F}, \quad C_{F}:=\frac{\left(2 c_{f} C_{f}\right)^{q}}{q}
$$

and

$$
\mathcal{B}(m, \boldsymbol{v})=\int_{Q} L(x, \boldsymbol{v}) m+F(x, m) \mathrm{d} x \mathrm{~d} t \geq \int_{Q}\left(\frac{1}{2 c_{H}} m|\boldsymbol{v}|^{2}+\frac{1}{2 p c_{f}^{p}} m^{p}\right) \mathrm{d} x \mathrm{~d} t-\left(C_{F}+c_{H}^{+} M\right)
$$

Setting $c:=2 \max \left(c_{H}, p c_{f}^{p}\right)$, we conclude that

$$
\int_{Q}\left(m|\boldsymbol{v}|^{2}+m^{p}\right) \mathrm{d} x \mathrm{~d} r \leq E:=c\left(B+C_{F}+c_{H}^{+} M\right) .
$$

We eventually obtain a bound of $\boldsymbol{w}=m \boldsymbol{v}$ in $L^{2 p /(p+1)}(Q)$ by

$$
\|m \boldsymbol{v}\|_{L^{2 p /(p+1)}(Q)} \leq\left\|m^{1 / 2}\right\|_{L^{2 p}(Q)}\left\|m^{1 / 2} \boldsymbol{v}\right\|_{L^{2}\left(Q ; \mathbb{R}^{d}\right)}=\|m\|_{L^{p}(Q)}^{1 / 2}\left(\int_{Q}|\boldsymbol{v}|^{2} m \mathrm{~d} x \mathrm{~d} t\right)^{1 / 2} \leq E .
$$

(6.5) shows that for $(m, \boldsymbol{v}) \in \mathrm{CE}_{2, p}\left(Q ; \mu_{0}, \mu_{1}\right)$ an upper bound of $\mathcal{B}(m, \boldsymbol{v})$ provides an upper bound for $K L_{2, p}\left(\mu_{0}, \mu_{1}\right)$. The converse property also holds.

Lemma 6.3 (A priori estimates II) Let us consider

$$
\mu_{i} \in \mathcal{P}_{2}\left(\mathbb{R}^{d}\right) \quad \text { with } \quad \int_{\mathbb{R}^{d}}|x|^{2} \mathrm{~d} \mu_{i}=M_{i}, \quad K L_{2, p}\left(\mu_{0}, \mu_{1}\right)=K<\infty .
$$

Then the infimum of $\mathcal{B}$ on $\mathrm{CE}_{2, p}\left(Q ; \mu_{0}, \mu_{1}\right)$ is finite and there are constants $B$ and $M$ only depending on $K, M_{i}$ and on the structural constants of (6.4) such that the infimum can be restricted to all the pairs $(m, \boldsymbol{v}) \in \mathrm{CE}_{2, p}\left(Q ; \mu_{0}, \mu_{1}\right)$ satisfying the apriori bounds (6.2) and (6.3); in particular, (m, v) also satisfy (6.5).

Proof. Let us pick a curve $(\bar{m}, \overline{\boldsymbol{v}}) \in \mathrm{CE}_{2, p}\left(Q ; \mu_{0}, \mu_{1}\right)$ with $\int_{Q}\left(|\overline{\boldsymbol{v}}|^{2} \bar{m}+\bar{m}^{p}\right) \mathrm{d} \lambda \leq 4 K$. We get the bound

$$
\int_{Q} F(x, \bar{m}) \mathrm{d} x \mathrm{~d} t \leq C_{1}:=4 \frac{c_{f}^{p}}{p} K+C_{f}(4 K)^{1 / p},
$$

thanks to the growth conditions (2.15). Since

$$
\left(\int_{\mathbb{R}^{d}}|x|^{2} \mathrm{~d} \bar{\mu}_{t}\right)^{1 / 2} \leq M_{0}^{1 / 2}+W_{2}\left(\mu_{t}, \mu_{0}\right) \leq M_{0}^{1 / 2}+(2 K)^{1 / 2},
$$


we also get

$$
\int_{0}^{1} \int_{\mathbb{R}^{d}} L(x, \overline{\boldsymbol{v}}) \bar{m} \mathrm{~d} x \mathrm{~d} t \leq C_{2}:=c_{H} K+3 c_{H}^{-}\left(1+M_{0}+2 K\right)
$$

thanks to (2.12), (2.20). In particular $\mathcal{B}(\bar{m}, \overline{\boldsymbol{v}}) \leq B:=C_{1}+C_{2}$.

We now fix an element $(m, \boldsymbol{v}) \in \mathrm{CE}_{2, p}\left(Q ; \mu_{0}, \mu_{1}\right)$ with $\boldsymbol{w}=m \boldsymbol{v}$ satisfying $\mathcal{B}(m, \boldsymbol{v}) \leq B$, and we introduce the absolutely continuous function

$$
t \mapsto M(t):=\left(\int_{\mathbb{R}^{d}}|x|^{2} \mathrm{~d} \mu_{t}\right)^{1 / 2} \geq \int_{\mathbb{R}^{d}}|x| \mathrm{d} \mu_{t}, \quad t \in[0,1],
$$

whose derivative satisfies

$$
\left|\frac{\mathrm{d}}{\mathrm{d} t} M(t)\right| \leq\left(\int_{\mathbb{R}^{d}}\left|\boldsymbol{v}_{t}\right|^{2} \mathrm{~d} \mu_{t}\right)^{1 / 2}, \quad\left|\frac{\mathrm{d}}{\mathrm{d} t} M^{2}(t)\right| \leq M^{2}(t)+\int_{\mathbb{R}^{d}}\left|\boldsymbol{v}_{t}\right|^{2} \mathrm{~d} \mu_{t} \quad \text { a.e. in }(0,1) .
$$

Arguing as for (6.7) we obtain for a.e. $t \in(0,1)$

$$
B(t):=\int_{\mathbb{R}^{d}} L\left(x, \boldsymbol{v}_{t}\right) m_{t}+F\left(x, m_{t}\right) \mathrm{d} x \geq \int_{\mathbb{R}^{d}}\left(\frac{1}{2 c_{H}} m_{t}\left|\boldsymbol{v}_{t}\right|^{2}+\frac{1}{2 p c_{f}^{p}} m_{t}^{p}\right) \mathrm{d} x-C_{F}-c_{H}^{+} M(t) .
$$

By 6.15 we deduce that

$$
\left|\frac{\mathrm{d}}{\mathrm{d} t} M^{2}(t)\right| \leq 2 c_{H}\left(B(t)+C_{3}\right)+2 M^{2}(t), \quad C_{3}:=C_{F}+\frac{1}{2}\left(c_{H}^{+}\right)^{2}
$$

so that a simple comparison argument yields the two estimates

$$
M^{2}(t) \leq\left(M_{0}+2 c_{H} C_{3}+2 c_{H} \int_{0}^{t} B(r) \mathrm{d} r\right) \mathrm{e}^{2}, \quad M^{2}(t) \leq\left(M_{1}+2 c_{H} C_{3}+2 c_{H} \int_{t}^{1} B(r) \mathrm{d} r\right) \mathrm{e}^{2} .
$$

Summing up the previous inequalities we end up with

$$
1+M(t) \leq M:=1+\mathrm{e}\left(M_{0}+M_{1}+2 c_{H}\left(C_{3}+B\right)\right)^{1 / 2} \quad \text { for every } t \in[0,1]
$$

Remark 6.4 Notice that Lemma 3.5 yields a uniform estimate of the constants $B$ and $M$ of the previous Lemma in terms of

$$
M_{01}:=\max \left(\left\|m_{0}\right\|_{L^{p} \cap L_{\kappa}^{1}\left(\mathbb{R}^{d}\right)},\left\|m_{1}\right\|_{L^{p} \cap L_{\kappa}^{1}\left(\mathbb{R}^{d}\right)}\right) .
$$

We will now write Problem 6.1 in terms of a minimization of a convex functional on a convex set. To this aim, for every $(m, \boldsymbol{v}) \in \mathrm{CE}_{2, p}(Q)$ we introduce the functions

$$
\boldsymbol{w}:=m \boldsymbol{v} \in L^{\frac{2 p}{p+1}}\left(Q ; \mathbb{R}^{d}\right), \quad \tilde{L}(x, m, \boldsymbol{w}):= \begin{cases}L(x, \boldsymbol{w} / m) m & \text { if } m \neq 0, \\ +\infty & \text { if } m=0, \boldsymbol{w} \neq 0 \\ 0 & \text { if } m=0, \boldsymbol{w}=0\end{cases}
$$

and write $\mathcal{B}$ as a functional of the pair $(m, \boldsymbol{w})$ :

$$
\tilde{\mathcal{B}}(m, \boldsymbol{w}):=\int_{0}^{1} \int_{\mathbb{R}^{d}}(\tilde{L}(x, m(t, x), \boldsymbol{w}(t, x))+F(x, m(t, x))) \mathrm{d} x \mathrm{~d} t .
$$

Thanks to the coercivity conditions of $L$, if $m \in L^{p} \cap L_{\kappa}^{1}(Q), \boldsymbol{w} \in L^{1}\left(Q ; \mathbb{R}^{d}\right)$ and $\tilde{\mathcal{B}}(m, \boldsymbol{w})<\infty$, then we can write $\boldsymbol{w}=m \boldsymbol{v}$ for a vector field $\boldsymbol{v} \in L^{2}\left(Q, \tilde{\mu} ; \mathbb{R}^{d}\right)$. If moreover $(m, \boldsymbol{w})$ satisfy the conservation law

$$
\partial_{t} m+\nabla \cdot \boldsymbol{w}=0 \quad \text { in } \mathscr{D}^{\prime}(Q)
$$

then $(m, \boldsymbol{v}) \in \mathrm{CE}_{2, p}(Q)$ so that $m$ admits a continuous representative $\mu \in \mathrm{AC}^{2}\left([0,1] ; \mathcal{P}_{2}\left(\mathbb{R}^{d}\right)\right)$ and the initial and final conditions $\mu_{t=i}=\mu_{i}, i=0,1$, make sense.

Therefore Problem 6.1 is in fact equivalent to the minimization of the convex functional $\tilde{\mathcal{B}}$ on the convex set $\mathbb{K}$ depending on $\mu_{0}, \mu_{1}$ :

$$
\begin{gathered}
\mathbb{K}:=\left\{(m, \boldsymbol{w}) \in\left(L^{p} \cap L_{\kappa}^{1}(Q)\right) \times L^{2 p /(p+1)}\left(Q ; \mathbb{R}^{d}\right): \partial_{t} m+\nabla \cdot \boldsymbol{w}=0 \quad \text { in } \mathscr{D}^{\prime}(Q),\right. \\
\left.m \lambda=\tilde{\mu} \quad \text { for a curve } \mu \in \mathrm{AC}^{2}\left([0,1] ; \mathcal{P}_{2}\left(\mathbb{R}^{d}\right)\right), \quad \mu_{t=0}=\mu_{0}, \mu_{t=1}=\mu_{1}\right\} .
\end{gathered}
$$


Lemma 6.5 (Lower semicontinuity of $\tilde{\mathcal{B}})$ If $\left(m_{n}, \boldsymbol{w}_{n}\right)$ is a sequence weakly converging to $(m, \boldsymbol{w})$ in $L^{p}(Q) \times L^{2 p /(p+1)}\left(Q ; \mathbb{R}^{d}\right)$ and satisfyng $m_{n} \geq 0, \int_{Q}\left(1+|x|^{2}\right) m_{n} \mathrm{~d} x \mathrm{~d} t \leq M$, then for every choice of $g, \boldsymbol{g}, h$ measurable functions $\boldsymbol{g}: Q \rightarrow \mathbb{R}^{d}, g: Q \rightarrow \mathbb{R}, h: Q \rightarrow \mathbb{R}$ with

$$
|g(t, x)| \leq C_{g}(1+|x|), \quad \boldsymbol{g} \in L^{\infty}\left(Q ; \mathbb{R}^{d}\right), \quad h \in L^{q}(Q),
$$

we have

$$
\liminf _{n \rightarrow \infty} \tilde{\mathcal{B}}\left(m_{n}, \boldsymbol{w}_{n}\right)+\int_{Q}\left((g+h) m_{n}+\boldsymbol{g} \cdot \boldsymbol{w}_{n}\right) \mathrm{d} \lambda \geq \tilde{\mathcal{B}}(m, \boldsymbol{w})+\int_{Q}((g+h) m+\boldsymbol{g} \cdot \boldsymbol{w}) \mathrm{d} \lambda .
$$

In particular

$$
\liminf _{n \rightarrow \infty} \tilde{\mathcal{B}}\left(m_{n}, \boldsymbol{w}_{n}\right) \geq \tilde{\mathcal{B}}(m, \boldsymbol{w})
$$

Proof. We can decompose $\tilde{\mathcal{B}}$ in the sum

$$
\tilde{\mathcal{B}}(m, \boldsymbol{w})=\tilde{\mathcal{B}}_{1}(m, \boldsymbol{w})-\int_{Q}\left(\gamma_{H}^{+}(x)+\gamma_{f}(x)\right) m \mathrm{~d} x \mathrm{~d} t,
$$

where $\tilde{\mathcal{B}}_{1}$ is defined as $\tilde{\mathcal{B}}$ starting from the integrands $F_{1}(x, m):=F(x, m)+\gamma_{f}(x) m$ and $\tilde{L}_{1}(x, m, \boldsymbol{w}):=$ $\tilde{L}(x, m, \boldsymbol{w})+\left(\gamma_{H}^{+}(x)\right) m$. Since $F_{1}$ and $\tilde{L}_{1}$ are nonnegative, measurable, convex and lower semicontinuous with respect to the variables $m$ and $(m, \boldsymbol{w})$ respectively, $\tilde{\mathcal{B}}_{1}$ is weakly lower semicontinuous in $L^{p}(Q) \times$ $L^{2 p /(p+1)}\left(Q ; \mathbb{R}^{d}\right)$ by the general result $[17$, Theorem 6.54$]$.

Since we can incorporate the contribution of $\gamma_{H}^{+}$in $g$ and the contribution of $\gamma_{f}$ in $h$, it remains to check that

$$
\liminf _{n \rightarrow \infty} \int_{Q}\left((g+h) m_{n}+\boldsymbol{g} \cdot \boldsymbol{w}_{n}\right) \mathrm{d} \lambda \geq \int_{Q}((g+h) m+\boldsymbol{g} \cdot \boldsymbol{w}) \mathrm{d} \lambda
$$

where $g \leq 0$. Weak convergence in $L^{p}(Q)$ yields $\lim _{n \rightarrow \infty} \int_{Q} h m_{n} \mathrm{~d} \lambda=\int_{Q} h m \mathrm{~d} \lambda$. The contribution of $\boldsymbol{g}$ can also be controlled since $\boldsymbol{w}_{n}$ is weakly converging to $\boldsymbol{w}$ in $L^{1}\left(Q ; \mathbb{R}^{d}\right)$.

Concerning the third term arising from the integral against $g$, we use the the uniform estimate $\int_{Q}(1+$ $\left.|x|^{2}\right) m_{n} \mathrm{~d} x \mathrm{~d} t \leq M$ and for every $\delta \in(0,1 / 2)$

$$
-g(t, x) \leq C\left(\delta^{-1}+\delta|x|^{2}\right), \quad \mathscr{L}^{d} \text {-a.e. in } \mathbb{R}^{d} .
$$

For every $\delta>0$ choosing and $K_{\delta}:=\left\{x \in \mathbb{R}^{d}:|x| \leq \delta^{-1}\right\}$ we get

$$
\begin{aligned}
\liminf _{n \rightarrow \infty} \int_{Q} g m_{n} \mathrm{~d} \lambda & \geq \liminf _{n \rightarrow \infty} \int_{0}^{1} \int_{\mathbb{R}^{d} \backslash K_{\delta}} g m_{n} \mathrm{~d} x \mathrm{~d} t+\liminf _{n \rightarrow \infty} \int_{0}^{1} \int_{K_{\delta}} g m_{n} \mathrm{~d} x \mathrm{~d} t \\
& \geq-C \liminf _{n \rightarrow \infty} \int_{0}^{1} \int_{\mathbb{R}^{d} \backslash K_{\delta}}\left(\delta^{-1}+\delta|x|^{2}\right) m_{n} \mathrm{~d} x \mathrm{~d} t+\int_{0}^{1} \int_{K_{\delta}} g m \mathrm{~d} x \mathrm{~d} t \\
& \geq-2 C \delta M+\int_{0}^{1} \int_{K_{\delta}} g m \mathrm{~d} x \mathrm{~d} t .
\end{aligned}
$$

Since $\delta>0$ is arbitrary, we conclude.

Theorem 6.6 For every choice of $\mu_{i}$ as in (6.10) Problem 6.1 admits a solution $\left(m_{\star}, \boldsymbol{v}_{\star}\right)$, and $\left(m_{\star}, m_{\star} \boldsymbol{v}_{\star}\right)$ is a minimizer of $\tilde{\mathcal{B}}$ on $\mathbb{K}$.

Moreover, if the map $m \mapsto f(x, m)$ is strictly increasing in $\mathbb{R}$ for a.e. $x \in \mathbb{R}^{d}$, then the minimizer $m$ is unique and the optimal vector field $\boldsymbol{v}$ is unique $m \lambda$-a.e. in $Q$.

Proof. By the previous Lemmas 6.3 and 6.2, we can minimize $\tilde{\mathcal{B}}$ among all curves $(m, \boldsymbol{w}) \in \mathbb{K}, \boldsymbol{w}=m \boldsymbol{v}$, satisfying the apriori bounds (6.2), (6.3) and therefore (6.5). The existence of a minimizer then follows by the direct method of the Calculus of Variations. If $\left(m_{n}, \boldsymbol{v}_{n}\right) \in \mathrm{CE}_{2, p}\left(Q ; \mu_{0}, \mu_{1}\right)$ is a minimizing sequence for $\mathcal{B}$, we easily see from (6.5) that $m_{n}$ is uniformly bounded in $L^{p}(Q)$ and $\boldsymbol{w}_{n}=m_{n} \boldsymbol{v}_{n}$ is uniformly bounded in $L^{2 p /(p+1)}\left(Q ; \mathbb{R}^{d}\right)$. The curves $t \mapsto \mu_{n, t}$ are equi uniformly continuous, since

$$
W_{2}\left(\mu_{n, t}, \mu_{n, s}\right) \leq|t-s|^{1 / 2}\left(\int_{0}^{T}\left|\boldsymbol{v}_{n}\right|^{2} m_{n} \mathrm{~d} x \mathrm{~d} t\right)^{1 / 2}
$$

up to extracting a suitable subsequence, we can thus assume that $m_{n} \rightarrow m$ in $L^{p}(Q), \boldsymbol{w}_{n} \rightarrow \boldsymbol{w}=m \boldsymbol{v}$ in $L^{2 p /(p+1)}\left(Q ; \mathbb{R}^{d}\right)$ and $\mu_{n, t} \rightarrow \mu_{t}$ weakly in $\mathcal{P}\left(\mathbb{R}^{d}\right)$ for every $t \in[0,1]$, with $(m, \boldsymbol{v}) \in \mathrm{CE}_{2, p}\left(Q ; \mu_{0}, \mu_{1}\right) ;$ the pair $(m, \boldsymbol{w})$ thus belong to $\mathbb{K}$ (in particular it satisfies the initial and final conditions).

We can then apply the lower semicontinuity of $\tilde{\mathcal{B}}$ given by Lemma 6.5. The uniqueness of minimizer easily follows by the strict convexity of the map $m \mapsto F(x, m)$. Once $m$ is uniquely determined, the strict convexity of $L(x, \cdot)$ (guaranteed by the $\boldsymbol{p}$-differentiability of $H$ ) ensures the uniqueness of $\boldsymbol{v}$. 


\subsection{The variational structure of the dual problem and the minimax principle}

In this section we will compute and study the dual formulation of Problem 6.1.

Problem 6.7 Let $\mu_{i}=m_{i} \mathscr{L}^{d} \in \mathcal{P}_{2, p}^{r}\left(\mathbb{R}^{d}\right)$ be given. We look for the maximizer of the functional

$$
\mathcal{A}(u, \alpha):=\int_{\mathbb{R}^{d}} u^{+}(0, x) \mathrm{d} \mu_{0}(x)-\int_{\mathbb{R}^{d}} u^{-}(1, x) \mathrm{d} \mu_{1}(x)-\int_{0}^{1} \int_{\mathbb{R}^{d}} F^{*}(x, \alpha(t, x)) \mathrm{d} x \mathrm{~d} t
$$

among all pairs $(u, \alpha) \in \operatorname{HJ}_{q}(Q, H)$ according to Definition 5.7 .

Notice that by Theorem 5.10 the functional $\mathcal{A}$ is well defined and takes values in $[-\infty,+\infty)$. Corollary 5.15 also shows that

$$
\mathcal{A}(u, \alpha) \leq \mathcal{B}(m, \boldsymbol{v}) \quad \text { for every }(u, \alpha) \in \mathrm{HJ}_{q}(Q, H), \quad(m, \boldsymbol{v}) \in \mathrm{CE}_{2, p}\left(Q ; \mu_{0}, \mu_{1}\right),
$$

so that

$$
\sup \left\{\mathcal{A}(u, \alpha):(u, \alpha) \in \operatorname{HJ}_{q}(Q, H)\right\} \leq \min \left\{\mathcal{B}(m, \boldsymbol{v}):(m, \boldsymbol{v}) \in \mathrm{CE}_{2, p}\left(Q ; \mu_{0}, \mu_{1}\right)\right\} .
$$

We will present two main results; first of all, we will justify the duality between Problem 6.7 and Problem 6.1 by a classical min-max argument starting from a suitable saddle point formulation: in this way we will show that there is no duality gap in (6.32), even if we restrict the set of competitors for the dual problem to smoother functions. This weak duality result holds for arbitrary pair of measures $\mu_{0}, \mu_{1} \in \mathcal{P}_{2}\left(\mathbb{R}^{d}\right)$.

Our second result will show that if $\mu_{i}$ have densities in $L^{p}$ then also Problem 6.7 admits a solution and the supremum in (6.32) is attained.

We first compute the form of the dual problem by a classical min-max argument.

\section{Saddle point formulation of primal and dual problems}

Let us consider the convex set

$$
\mathbb{B}:=\left\{(m, \boldsymbol{w}) \in\left(L^{p} \cap L_{\kappa}^{1}(Q)\right) \times L^{2 p /(p+1)}\left(Q ; \mathbb{R}^{d}\right): m \geq 0, \tilde{\mathcal{B}}(m, \boldsymbol{w})<\infty\right\} .
$$

A pair $(m, \boldsymbol{w}) \in \mathbb{B}$ is a weak solution of the continuity equation

$$
\partial_{t} m+\nabla \cdot \boldsymbol{w}=0 \quad \text { in } \mathscr{D}^{\prime}(Q)
$$

with initial and final condition $\mu_{0}, \mu_{1} \in \mathcal{P}_{2}\left(\mathbb{R}^{d}\right)$ if and only if

$$
\sup _{u \in \mathbb{A}} \int_{\mathbb{R}^{d}} u_{0} \mathrm{~d} \mu_{0}-\int_{\mathbb{R}^{d}} u_{1} \mathrm{~d} \mu_{1}+\int_{Q}\left(\partial_{t} u m+D u \cdot \boldsymbol{w}\right) \mathrm{d} x \mathrm{~d} t=0,
$$

where

$$
\mathbb{A}:=\left\{u \in C^{1}(\bar{Q}): \sup _{Q} \frac{|u|+\left|\partial_{t} u\right|}{1+|x|}+|D u|<+\infty\right\} .
$$

Notice that when $u \in \mathbb{A}$ and $\alpha:=-\partial_{t} u+H(x, D u)$ (6.30) still makes sense for arbitrary measures $\mu_{0}, \mu_{1} \in$ $\mathcal{P}_{2}\left(\mathbb{R}^{d}\right)$. By introducing the saddle function

$$
\begin{aligned}
\mathcal{L}((m, \boldsymbol{w}), u):= & \int_{Q}(\tilde{L}(x, m, \boldsymbol{w})+F(x, m)) \mathrm{d} x \mathrm{~d} t \\
& +\int_{\mathbb{R}^{d}} u_{0} \mathrm{~d} \mu_{0}-\int_{\mathbb{R}^{d}} u_{1} \mathrm{~d} \mu_{1}+\int_{Q}\left(\partial_{t} u m+D u \cdot \boldsymbol{w}\right) \mathrm{d} x \mathrm{~d} t
\end{aligned}
$$

the primal problem can be equivalently obtained as

$$
\min _{(m, \boldsymbol{w}) \in \mathbb{K}} \tilde{\mathcal{B}}(m, \boldsymbol{w})=\min _{(m, \boldsymbol{w}) \in \mathbb{B}} \sup _{u \in \mathbb{A}} \mathcal{L}((m, \boldsymbol{w}), u) .
$$

Notice that $\mathcal{L}$ is convex w.r.t. $(m, \boldsymbol{w})$ and concave w.r.t. $(u, \alpha)$. A nice application of Von Neumann Theorem yields the following result:

Theorem 6.8 For every $\mu_{0}, \mu_{1} \in \mathcal{P}_{2}\left(\mathbb{R}^{d}\right)$ we have

$$
\begin{aligned}
\min _{(m, \boldsymbol{w}) \in \mathbb{K}} \tilde{\mathcal{B}}(m, \boldsymbol{w}) & =\min _{(m, \boldsymbol{w}) \in \mathbb{B}} \sup _{u \in \mathbb{A}} \mathcal{L}((m, \boldsymbol{w}), u)=\sup _{u \in \mathbb{A}} \inf _{(m, \boldsymbol{w}) \in \mathbb{B}} \mathcal{L}((m, \boldsymbol{w}), u) \\
& =\sup \left\{\mathcal{A}(u, \alpha): u \in \mathbb{A},-\partial_{t} u+H(x, D u) \leq \alpha, \alpha \in \mathcal{X}^{q}(Q)\right\} .
\end{aligned}
$$


Proof. Let us first check that we can apply Von Neumann minimax Theorem A.1. Clearly $\mathcal{L}$ satisfies A.1 and (A.2) and it is not restrictive to assume that $C_{\star}:=1+\sup _{u \in \mathbb{A}} \inf _{(m, \boldsymbol{w}) \in \mathbb{B}} \mathcal{L}((m, \boldsymbol{w}), u)$ is finite. We endow $\mathbb{B}$ with the product weak topology of $L^{p}(Q) \times L^{2 p /(p+1)}(Q)$.

We choose constants $A_{1} \geq 1+c_{H}^{+}$and $A_{0} \geq 1+4 c_{H} A_{1}+c_{H}^{+}$and the function $u_{\star}:=2 t\left(A_{0}^{2}+A_{1}^{2}|x|^{2}\right)^{1 / 2} \in \mathbb{A}$; we can check that

$$
\begin{aligned}
\partial_{t} u_{\star}+D u_{\star} \cdot \boldsymbol{v} & \geq 2\left(A_{0}^{2}+A_{1}^{2}|x|^{2}\right)^{1 / 2}-2 A_{1}|\boldsymbol{v}| \geq 2\left(A_{0}^{2}+A_{1}^{2}|x|^{2}\right)^{1 / 2}-4 c_{H} A_{1}-\frac{1}{4 c_{H}}|\boldsymbol{v}|^{2}, \\
L(x, \boldsymbol{v})+\partial_{t} u_{\star}+D u_{\star} \cdot \boldsymbol{v} & \geq \frac{1}{4 c_{H}}|\boldsymbol{v}|^{2}+2\left(A_{0}^{2}+A_{1}^{2}|x|^{2}\right)^{1 / 2}-4 c_{H} A_{1}-c_{H}^{+}(1+|x|) \\
& \geq \frac{1}{4 c_{H}}|\boldsymbol{v}|^{2}+\left(A_{0}-4 c_{H} A_{1}-c_{H}^{+}\right)+\left(A_{1}-c_{H}^{+}\right)|x| \geq \frac{1}{4 c_{H}}|\boldsymbol{v}|^{2}+(1+|x|),
\end{aligned}
$$

so that

$$
\mathcal{L}\left((m, \boldsymbol{w}), u_{\star}\right) \geq \int_{Q}\left(\frac{1}{4 c_{H}} m|\boldsymbol{v}|^{2}+\frac{1}{2 p c_{f}^{p}} m^{p}+(1+|x|) m\right) \mathrm{d} x \mathrm{~d} t-C_{\mathcal{L}},
$$

with $C_{\mathcal{L}}:=2 A_{0}+2 A_{1} \int_{\mathbb{R}^{d}}|x| \mathrm{d} \mu_{1}+C_{F}$.

Combining (6.40) with the estimate (6.9), we can see that the convex set

$$
\mathbb{B}_{\star}:=\left\{(m, \boldsymbol{w}) \in \mathbb{B}: \mathcal{L}\left((m, \boldsymbol{w}), u_{\star}\right) \leq C_{\star}\right\}
$$

is compact in $\mathbb{B}$. Lemma 6.5 also shows that for every $u \in \mathbb{A}(m, \boldsymbol{w}) \mapsto \mathcal{L}((m, \boldsymbol{w}), u)$ is lower semicontinuous in $\mathbb{B}_{\star}$ so that we can interchange the order inf and sup in (6.39).

Let us now compute

$$
\begin{aligned}
\inf _{(m, \boldsymbol{w}) \in \mathbb{B}} \mathcal{L}((m, \boldsymbol{w}), u)=\int_{\mathbb{R}^{d}} u_{0} \mathrm{~d} \mu_{0}-\int_{\mathbb{R}^{d}} u_{1} \mathrm{~d} \mu_{1}-\mathcal{C}(u) \\
\mathcal{C}(u):=\sup _{(m, \boldsymbol{w}) \in \mathbb{B}} \int_{Q}\left(-\partial_{t} u m-D u \cdot \boldsymbol{w}\right) \mathrm{d} x \mathrm{~d} t-\int_{Q}(\tilde{L}(x, m, \boldsymbol{w})+F(x, m)) \mathrm{d} x \mathrm{~d} t \\
=\sup _{m \in L^{p} \cap L_{\kappa}^{1}(Q), m \geq 0}\left(\int_{Q}\left(-\partial_{t} u m-F(x, m)\right) \mathrm{d} x \mathrm{~d} t\right. \\
\left.\quad+\sup _{\boldsymbol{v} \in L^{2}\left(Q, m \lambda ; \mathbb{R}^{d}\right)} \int_{Q}(-D u \cdot \boldsymbol{v}-L(x, \boldsymbol{v})) m \mathrm{~d} x \mathrm{~d} t\right)
\end{aligned}
$$

By applying the general duality theorem [16, Chap. IX, Prop. 2.1] (see also Remark 2.1) to the space $L^{2}\left(Q, m \lambda ; \mathbb{R}^{d}\right)$ (notice that $m$ is a finite measure and $D u \in L^{2}\left(Q, m \lambda ; \mathbb{R}^{d}\right)$ ) we obtain

$$
\sup _{\boldsymbol{v} \in L^{2}\left(Q, m \lambda ; \mathbb{R}^{d}\right)} \int_{Q}(D u \cdot \boldsymbol{v}-L(x, \boldsymbol{v})) m \mathrm{~d} x \mathrm{~d} t=\int_{Q} H(x, D u) m \mathrm{~d} x \mathrm{~d} t .
$$

Let us now set $\alpha:=-\partial_{t} u+H(x, D u) \in L_{1 / \kappa}^{\infty}(Q)$ we obtain

$$
\mathcal{C}(u)=\sup _{m \in L^{p} \cap L_{\kappa}^{1}(Q), m \geq 0} \int_{Q}(\alpha m-F(x, m)) \mathrm{d} x \mathrm{~d} t .
$$

Since $\alpha m-F(x, m) \leq F^{*}(x, \alpha)$ it is immediate to see that

$$
\mathcal{C}(u) \leq \int_{Q} F^{*}(x, \alpha) \mathrm{d} x \mathrm{~d} t
$$

On the other hand, restricting the supremum in (6.42) to functions $m$ vanishing outside a rectangle $R_{k}=$ $(0,1) \times B_{k}$, where $B_{k}$ is the ball centered at 0 of radious $k$ in $\mathbb{R}^{d}$, and applying the [16, Chap. IX, Prop. $2.1]$ in $L^{p}\left(R_{k}\right)$ we obtain

$$
\mathcal{C}(u) \geq \int_{R_{k}} F^{*}(x, \alpha) \mathrm{d} x \mathrm{~d} t
$$

Since $F^{*}$ is nonnegative, a limit as $k \rightarrow \infty$ by Beppo Levi monotone convergence theorem yields

$$
\mathcal{C}(u)=\int_{Q} F^{*}(x, \alpha) \mathrm{d} x \mathrm{~d} t
$$

Eventually, since $F^{*}$ is increasing w.r.t. the second variable, we obtain the last identity of (6.39). 


\subsection{Existence of a solution to the dual problem}

Let us now show that in Problem 6.7 the maximum is reached and therefore it coincides with the supremum of Problem 6.7 when the measures $\mu_{i}$ have $L^{p}$ densities. Let us first make a preliminary remark concerning a natural lower bound for $\alpha$.

Remark 6.9 (Lower bound on $\alpha$ ) Since $F^{*}(x, a) \geq 0$ and $F^{*}(x, a)=0$ if $a \leq f(x, 0)$, it is not restrictive to assume that any competing pair $(u, \alpha) \in \operatorname{HJ}_{q}(Q, H)$ for the maximization of $\mathcal{A}$ satisfies the lower bound

$$
\alpha(t, x) \geq f(x, 0) \quad \text { a.e. in } Q \text {. }
$$

In fact, if $(u, \alpha) \in \operatorname{HJ}_{q}(Q, H)$ we can always replace $\alpha$ by $\tilde{\alpha}:=\alpha \vee f(x, 0)$ still obtaining a pair $(u, \tilde{\alpha}) \in$ $\mathrm{HJ}_{q}(Q, H)$ with $\mathcal{A}(u, \alpha)=\mathcal{A}(u, \tilde{\alpha})$.

Theorem 6.10 (Solution to the dual problem) If $\mu_{i}=m_{i} \mathscr{L}^{d} \in \mathcal{P}_{2, p}^{r}\left(\mathbb{R}^{d}\right)$ then there exists an optimal pair $\left(u^{*}, \alpha^{*}\right) \in \operatorname{HJ}_{q}(Q, H)$ satisfying (6.46) such that

$$
\mathcal{A}\left(u^{*}, \alpha^{*}\right)=\max _{(u, \alpha) \mathrm{HJ}_{q}(Q, H)} \mathcal{A}(u, \alpha)
$$

Proof. Let $\left(u_{n}, \alpha_{n}\right) \in \mathrm{HJ}_{q}(Q, H)$ be a maximizing sequence such that

$$
\lim _{n \rightarrow \infty} \mathcal{A}\left(u_{n}, \alpha_{n}\right)=\sup _{(u, \alpha) \in \mathrm{HJ}_{q}(Q, H)} \mathcal{A}(u, \alpha)=A=\min _{(m, \boldsymbol{w}) \in \mathbb{K}} \tilde{\mathcal{B}}(m, \boldsymbol{w}) .
$$

We can assume that $\mathcal{A}\left(u_{n}, \alpha_{n}\right)$ is bounded below, say by $A-1$.

By remark 6.9 we can suppose that $\alpha_{n} \geq f(x, 0) \geq-\gamma_{f}$. (2.17) also yields

$$
\frac{1}{q c_{f}^{q}} \int_{Q}\left(\alpha_{n}-\gamma_{f}\right)_{+}^{q} \mathrm{~d} x \mathrm{~d} t \leq \int_{Q} F^{*}\left(x, \alpha_{n}\right) \mathrm{d} x \mathrm{~d} t \leq \int_{\mathbb{R}^{d}} u_{n}^{+}(0, x) m_{0} \mathrm{~d} x-\int_{\mathbb{R}^{d}} u_{n}^{-}(1, x) m_{1} \mathrm{~d} x+1-A .
$$

Using Corollary 5.13, we get that there exists a constant $C_{1}>0$ such that

$$
\int_{\mathbb{R}^{d}} u_{n}^{+}(0, x) m_{0} \mathrm{~d} x-\int_{\mathbb{R}^{d}} u_{n}^{-}(1, x) m_{1} \mathrm{~d} x \leq C_{1}\left(1+\left\|\alpha_{n}\right\|_{\mathcal{X}^{q}\left(\mathbb{R}^{d}\right)}\right) \quad \text { for every } n \in N .
$$

Combining this information with $\alpha_{n} \geq-\gamma_{f}$ and (6.47) this implies that

$$
\left\|\alpha_{n}\right\|_{\mathcal{X}^{q}(Q)}^{q} \leq C_{2}\left(1+\left\|\alpha_{n}\right\|_{\mathcal{X}^{q}(Q)}\right)
$$

whence the uniform bound $\left\|\alpha_{n}\right\|_{\mathcal{X}^{q}(Q)} \leq C_{3}$ and consequently $\int_{Q} F^{*}\left(x, \alpha_{n}\right) \leq C_{4}$, for constants $C_{i}$ independent of $n$.

Since $\mathcal{A}$ is invariant under translations of $u$ by a constant, we can tune $\left(u_{n}, \alpha_{n}\right)$ so that $\int_{\mathbb{R}^{d}} u_{n}^{-}(1, x) m_{1} \mathrm{~d} x=$ 0 . If we recall that $\int_{\mathbb{R}^{d}} u_{n}^{+}(0, x) m_{0} \mathrm{~d} x \geq \int_{Q} F^{*}\left(x, \alpha_{n}\right)+A-1$, we deduce the uniform estimate

$$
A-1 \leq \int_{\mathbb{R}^{d}} u_{n}^{+}(0, x) m_{0} \mathrm{~d} x \leq A+C_{4}
$$

Now, thanks to the uniform bound on $\left\|\alpha_{n}\right\|_{c X^{q}(Q)}$ there exists a subsequence $k \mapsto n(k)$ such that $\alpha_{n(k)} \rightarrow^{*} \alpha^{*}$ in $\mathcal{X}^{q}(Q)$. By Theorem 5.10 we also get for every $[a, b] \subset I$

$$
\sup _{n \in \mathbb{N}, r \in[a, b]}\left\|\mathbf{u}_{n}(r, \cdot)\right\|_{\mathcal{X}^{q}\left(\mathbb{R}^{d}\right)}<\infty .
$$

Then, from the stability result contained in Theorem 5.11 there exists a subsequence $\{n(k)\}$ and a limit function $u^{*}$ such that $\left(u^{*}, \alpha^{*}\right) \in \operatorname{HJ}_{q}(Q, H)$ and $\mathcal{A}\left(u^{*}, \alpha^{*}\right) \geq \lim \sup _{n \rightarrow \infty} \mathcal{A}\left(u_{n(k)}, \alpha_{n(k)}\right)=A$. Thus $\left(u^{*}, \alpha^{*}\right)$ attains the maximum of $\mathcal{A}$.

\subsection{Optimality conditions and the weak formulation of the Mean Field planning system}

Let us first derive the three crucial optimality conditions satisfied by the optimal solutions of the primal and of the dual problem. 
Theorem 6.11 (Optimality conditions) Let $\mu_{i}=m_{i} \mathscr{L}^{d} \in \mathcal{P}_{2, p}^{r}\left(\mathbb{R}^{d}\right)$ be given, let $(u, \alpha) \in \mathrm{HJ}_{q}(Q, H)$ be a weak subsolution to (5.1) satisfying (6.46), and let $(m, \boldsymbol{v}) \in \mathrm{CE}_{2, p}\left(Q ; \mu_{0}, \mu_{1}\right)$ be a weak solution to the continuity equation connecting $\mu_{0}$ to $\mu_{1}$. The following properties are equivalent:

(i) $\mathcal{A}(u, \alpha)=\mathcal{B}(m, \boldsymbol{v})$.

(ii) $(m, \boldsymbol{v})$ is an optimal solution to Problem 6.1, $(u, \alpha)$ is an optimal solution to Problem 6.7

(iii) $(u, \alpha)$ and $(m, \boldsymbol{v})$ satisfy the optimality conditions:

(O1) $\alpha=f(x, m) \lambda$-a.e. in $Q$.

(O2) $\boldsymbol{v}=-D_{p} H(x, D u) \tilde{\mu}$-a.e. in $Q$.

(O3) $u_{0}^{+} m_{0}, u_{1}^{-} m_{1} \in L^{1}\left(\mathbb{R}^{d}\right)$ and $u$ is a "renormalized" solution to

$$
\begin{cases}\partial_{t}(u m)+\nabla \cdot(u m \boldsymbol{v})=(H(x, D u)+D u \cdot \boldsymbol{v}-\alpha) m & \text { in } Q \\ (u m)_{t=0+}=u_{0}^{+} m_{0}, \quad(u m)_{t=1-}=u_{1}^{-} m_{1} & \text { on } \partial Q\end{cases}
$$

i.e. for every $(\zeta, Z) \in \mathscr{Z}_{c}$

$$
\partial_{t}(Z(u) m)+\nabla \cdot(Z(u) m \boldsymbol{v})=\zeta(u)(H(x, D u)+D u \cdot \boldsymbol{v}-\alpha) m \quad \text { in } \mathscr{D}^{\prime}(Q)
$$

and

$$
\underset{t \downarrow 0}{\operatorname{ap}-\lim } \int_{\mathbb{R}^{d}} u(t, x) m(t, x) \mathrm{d} x=\int_{\mathbb{R}^{d}} u_{0}^{+} m_{0} \mathrm{~d} x, \underset{t \uparrow 1}{\operatorname{ap}-\lim } \int_{\mathbb{R}^{d}} u(t, x) m(t, x) \mathrm{d} x=\int_{\mathbb{R}^{d}} u_{1}^{-} m_{1} \mathrm{~d} x .
$$

Proof. The equivalence between (i) and (ii) follows by (6.31) and Theorem 6.8. Corollary 5.17 yields the equivalence between (ii) and (iii).

Recall that (6.53) and (6.54) can be equivalently formulated as

$$
\begin{gathered}
-\int_{Q} Z(u) m\left(\partial_{t} \varphi+D \varphi \cdot \boldsymbol{v}\right) \mathrm{d} x \mathrm{~d} t+\int_{\mathbb{R}^{d}} Z\left(u_{0}^{+}\right) m_{0} \varphi(0, \cdot) \mathrm{d} x-\int_{\mathbb{R}^{d}} Z\left(u_{1}^{-}\right) m_{0} \varphi(0, \cdot) \mathrm{d} x= \\
=\int_{Q} \zeta(u)(H(x, D u)+D u \cdot \boldsymbol{v}-\alpha) m \varphi \mathrm{d} x \mathrm{~d} t \quad \text { for every } \varphi \in C_{c}^{1}(\bar{Q}) .
\end{gathered}
$$

The previous result provides a natural notion of solution to the system (r-MFPP), interpreted as the optimality condition for the two optimization problems 6.1 and 6.7 .

Definition 6.12 (Variational weak solutions to the MFPP system) Let $\mu_{i}=m_{i} \mathscr{L}^{d} \in \mathcal{P}_{2, p}^{r}\left(\mathbb{R}^{d}\right)$ be given and let us suppose that the structural assumption Assumptions 2.1] are satisfied. A pair $(m, u)$ is a weak solution to the MFPP system (r-MFPP) if

(i) $m \in L^{p} \cap L_{\kappa}^{1}(Q)$ is probability density and $u \in L_{\text {loc }}^{1}(Q)$ is weak subsolution to

$$
-\partial_{t} u+H(x, D u) \leq f(x, m) \quad \text { in } \mathscr{D}^{\prime}(Q) .
$$

In particular, the left and right traces $u_{0}^{+}, u_{1}^{-}$at $t=0,1$ are well defined in the sense of measure convergence of Theorem 5.10 .

(ii) $m$ belongs to $\mathrm{A}_{2}(Q)$ and it is a distributional solution to

$$
\partial_{t} m-\nabla \cdot\left(m D_{p} H(x, D u)\right)=0 \quad \text { in } \mathscr{D}^{\prime}(Q)
$$

In particular $m$ has a precise representative $\mu$ in the sense of Lemma 3.2 and left and right traces at $t=0,1$ satisfying the boundary conditions $\mu_{t=0}=\mu_{0}, \mu_{t=1}=\mu_{1}$.

(iii) $u_{0}^{+} m_{0}, u_{1}^{-} m_{1} \in L^{1}\left(\mathbb{R}^{d}\right)$ and $u$ is a "renormalized" solution to (6.52), in the sense of (6.53), (6.54).

Remark 6.13 Since $m \in \mathrm{A}_{2}(Q)$, it admits a precise representative $\mu \in \operatorname{AC}^{2}\left(I ; \mathcal{P}_{2}\left(\mathbb{R}^{d}\right)\right)$ so that the boundary conditions $\left.\mu\right|_{t=i}=\mu_{i}$ make sense, $m\left|D_{p} H(\cdot, D u)\right|^{2} \in L_{\text {loc }}^{1}(Q)$ and (6.57) can be formulated in the usual distributional sense, see Theorem 5.12

We can state Theorem 6.11 in the following form. 
Theorem 6.14 (Solutions to MFPP coincide with solutions to the primal-dual problem) Let us assume the structural conditions 2.1 with $\mu_{i}=m_{i} \mathscr{L}^{d} \in \mathcal{P}_{2, p}^{r}\left(\mathbb{R}^{d}\right)$.

1. If $(m, \boldsymbol{v}) \in \mathrm{CE}_{2, p}\left(Q ; \mu_{0}, \mu_{1}\right)$ is a minimizer for Problem 6.1 and $(u, \alpha) \in \mathrm{HJ}_{q}(H, Q)$ is a maximizer of Problem 6.7 also satisfying (6.46), then the pair $(m, u)$ is a variational weak solution of the system r-MFPP and we can identify $\alpha=f(\cdot, m) \mathscr{L}^{d+1}$-a.e. in $Q$ and $\boldsymbol{v}=-D_{p} H(\cdot, D u) \tilde{\mu}$-a.e. in $Q$.

2. Conversely, if $(m, u)$ is a variational weak solution to the planning problem (r-MFPP according to Definition 6.12, then the two pairs $\left(m,-D_{p} H(\cdot, D u)\right)$ and $(u, f(\cdot, m))$ are solutions to Problem 6.1 and Problem 6.7, respectively.

Existence of variational weak solutions to the MFPP system (r-MFPP can now be easily obtained by the results of Sections 6.1 and 6.2. As for uniqueness, this can only be obtained for $m$ and for $D u$ (on the support of $m$ ) by strengthening the convexity assumptions.

Theorem 6.15 (Existence and uniqueness of solutions to MFPP) Under the structural assumptions 2.1 with $\mu_{i}=m_{i} \mathscr{L}^{d} \in \mathcal{P}_{2, p}^{r}\left(\mathbb{R}^{d}\right)$ there exists a variational weak solution $(u, m)$ to the planning problem r-MFPP and the function

$$
\alpha:=f(x, m) \quad \text { is independent of the choice of } m,
$$

in the sense that if $(u, m)$ and $\left(u^{\prime}, m^{\prime}\right)$ are two solutions then $f(\cdot, m)=f\left(\cdot, m^{\prime}\right) \mathscr{L}^{d+1}$ a.e. in $Q$. Moreover

1. if $f(x, \cdot)$ is strictly increasing then $m$ is unique (up to $\mathscr{L}^{d+1}$-negligible sets) and the vector field $\boldsymbol{v}=$ $-D_{p} H(x, D u)$ is uniquely determined $m \lambda$-a.e. in $Q$

2. If $H$ is strictly convex (equivalently, if $L$ is differentiable w.r.t. $\boldsymbol{v}$ ) then also Du is uniquely determined $m \lambda$-a.e.

We conclude this section with a last characterization of optimizers of Problem 6.1, where a modified Lagrangian is involved. First of all, whenever $\alpha \in \mathcal{X}^{q}(Q)$ we set

$$
L_{\alpha}(t, x, \boldsymbol{v}):=L(x, \boldsymbol{v})+\alpha(t, x) .
$$

Theorem 6.16 (Optimizers of the modified Lagrangian cost) Let $\mu_{i}=m_{i} \mathscr{L}^{d} \in \mathcal{P}_{2, p}^{r}\left(\mathbb{R}^{d}\right)$ and $(m, \boldsymbol{v}) \in$ $\mathrm{CE}_{2, p}\left(Q ; \mu_{0}, \mu_{1}\right)$. The pair $(m, \boldsymbol{v})$ is a solution to Problem 6.1 if and only if setting $\alpha:=f(x, m)$ the pair $(m, \boldsymbol{v})$ minimizes the modified Lagrangian dynamic cost

$$
\mathcal{L}_{\alpha}\left(m^{\prime}, \boldsymbol{v}^{\prime}\right):=\int_{Q} L_{\alpha}\left(t, x, \boldsymbol{v}^{\prime}\right) m^{\prime} \mathrm{d} x \mathrm{~d} t=\int_{Q}\left(L\left(x, \boldsymbol{v}^{\prime}\right)+\alpha(t, x)\right) m^{\prime} \mathrm{d} x \mathrm{~d} t
$$

among all pairs $\left(m^{\prime}, \boldsymbol{v}^{\prime}\right) \in \mathrm{CE}_{2, p}\left(Q ; \mu_{0}, \mu_{1}\right)$, i.e.

$$
\mathcal{L}_{\alpha}(m, \boldsymbol{v}) \leq \mathcal{L}_{\alpha}\left(m^{\prime}, \boldsymbol{v}^{\prime}\right) \quad \text { for every }\left(m^{\prime}, \boldsymbol{v}^{\prime}\right) \in \mathrm{CE}_{2, p}\left(Q ; \mu_{0}, \mu_{1}\right)
$$

Proof. The sufficiency of (6.61) is easy: since $\alpha=f(x, m)$ we have by Fenchel inequality

$$
\alpha m=F(x, m)+F^{*}(x, \alpha), \quad \alpha m^{\prime} \leq F\left(x, m^{\prime}\right)+F^{*}(x, \alpha) \quad \lambda \text {-a.e. in } Q,
$$

so that (6.61) yields

$$
\begin{gathered}
\mathcal{B}(m, \boldsymbol{v})=\int_{Q}(L(x, \boldsymbol{v}) m+F(x, m)) \mathrm{d} \lambda=\mathcal{L}_{\alpha}(m, \boldsymbol{v})-\int_{Q} F^{*}(x, \alpha) \mathrm{d} \lambda \\
\stackrel{6.61}{\leq} \mathcal{L}_{\alpha}\left(m^{\prime}, \boldsymbol{v}^{\prime}\right)-\int_{Q} F^{*}(x, \alpha) \mathrm{d} \lambda \stackrel{\text { 6.62) }}{\leq} \mathcal{B}\left(m^{\prime}, \boldsymbol{v}^{\prime}\right) .
\end{gathered}
$$

In order to prove the converse implication, we use Theorem 6.10 to find a maximizer $\left(u, \alpha^{*}\right)$ of Problem 6.7 since $(m, \boldsymbol{v})$ is a solution to Problem 6.1. Theorem 6.14 shows that $\alpha^{*}=f(x, m)=\alpha$.

(6.62) and Theorem 5.12 (3) then yield

$$
\begin{aligned}
\mathcal{L}_{\alpha}(m, \boldsymbol{v}) & \stackrel{\sqrt{6.62})}{=} \mathcal{B}(m, \boldsymbol{v})+\int_{Q} F^{*}(x, \alpha) \mathrm{d} \lambda=\mathcal{A}(u, \alpha)+\int_{Q} F^{*}(x, \alpha) \mathrm{d} \lambda \\
& =\int_{\mathbb{R}^{d}} u_{0}^{+} \mathrm{d} \mu_{0}-\int_{\mathbb{R}^{d}} u_{1}^{-} \mathrm{d} \mu_{1} \leq \int_{Q}\left(\alpha-H(x, D u)-D u \cdot \boldsymbol{v}^{\prime}\right) m^{\prime} \mathrm{d} \lambda \\
& \leq \int_{Q}\left(\alpha+L\left(x, \boldsymbol{v}^{\prime}\right)\right) m^{\prime} \mathrm{d} \lambda=\mathcal{L}_{\alpha}\left(m^{\prime}, \boldsymbol{v}^{\prime}\right) .
\end{aligned}
$$


Notice that the function $\alpha$ defining the modified Lagrangian dynamic cost (6.60) does not depend on the particular choice of the solution $m$, thanks to (6.58). The above proof also shows that if $(m, \boldsymbol{v})$ is any optimal solution to Problem 6.1 and $(u, \alpha)$ is any optimal solution to Problem 6.7 we have

$$
\mathcal{L}_{\alpha}(m, \boldsymbol{v})=\int_{\mathbb{R}^{d}} u_{0}^{+} \mathrm{d} \mu_{0}-\int_{\mathbb{R}^{d}} u_{1}^{-} \mathrm{d} \mu_{1}
$$

so that these quantities do not depend on the particular choices of $m, \boldsymbol{v}$ and $u$ as well. It is natural to interpret the above results in terms of an Optimal Transport problem associated to the Lagrangian (6.59). This will be the aim of the next section.

\section{Optimal plans: a Lagrangian viewpoint}

In this section we are concerned with optimality conditions at the level of single agents trajectories. In particular we are interested in giving a good notion of Nash equilibria for the system. To do it we look at the problem in a Lagrangian fashion, studying suitable measures on admissible paths. The crucial step in this procedure is a probabilistic representation of measure-valued solutions to the continuity equation, known as superposition principle. A similar approach was already used for a Lagrangian formulation of mean field games in [13] borrowing arguments from [2], 10].

\subsection{Continuity equation and measures on the space of continuous curves}

We introduce the (complete and separable metric) space

$$
\Gamma:=C^{0}\left([0,1] ; \mathbb{R}^{d}\right) \quad \text { endowed with the uniform metric, }
$$

and its Borel (in fact $\left.F_{\sigma}\right)$ subset of absolutely continuous curves $\mathrm{AC}_{2}\left([0,1] ; \mathbb{R}^{d}\right)$; for every $\gamma \in \mathrm{AC}_{2}\left([0,1] ; \mathbb{R}^{d}\right)$ we call $\mathrm{E}_{2}$ the lower semicontinuous energy functional

$$
\mathrm{E}_{2}(\gamma):=\int_{0}^{1}|\dot{\gamma}(t)|^{2} \mathrm{~d} t, \quad \text { with } \quad \mathrm{E}_{2}(\gamma):=+\infty \text { if } \gamma \notin \mathrm{AC}_{2}\left([0,1] ; \mathbb{R}^{d}\right)
$$

We denote by $\mathrm{e}_{t}: \Gamma \rightarrow \mathbb{R}^{d}$ and $\mathrm{e}:[0,1] \times \Gamma \rightarrow Q$ the evaluation maps

$$
\mathrm{e}_{t}(\gamma):=\gamma(t), \quad \mathrm{e}(t, \gamma):=(t, \gamma(t)) \text { for every } t \in[0,1], \gamma \in \Gamma
$$

and by $\mathrm{d}:(0,1) \times \mathrm{AC}_{2}\left([0,1] ; \mathbb{R}^{d}\right) \rightarrow(0,1) \times \mathbb{R}^{d} \times \mathbb{R}^{d}$ the Borel map

$$
\mathrm{d}(t, \gamma):= \begin{cases}(t, \gamma(t), \dot{\gamma}(t)) & \text { if } \gamma \text { is differentiable at } t \\ (t, \gamma(t), 0) & \text { otherwise. }\end{cases}
$$

Every measure $\boldsymbol{\eta} \in \mathcal{P}(\Gamma)$ induces a continuous curve $\mu \in \mathrm{C}^{0}\left([0,1] ; \mathcal{P}\left(\mathbb{R}^{d}\right)\right)$ and a probability measure $\tilde{\mu} \in$ $\mathcal{P}(Q)$ by push-forward

$$
\mu_{t}:=\left(\mathrm{e}_{t}\right)_{\sharp} \boldsymbol{\eta}, \quad \tilde{\mu}=\mathrm{e}_{\sharp} \boldsymbol{\eta} .
$$

If $\boldsymbol{\eta}$ is concentrated on $\mathrm{AC}^{2}\left([0,1] ; \mathbb{R}^{d}\right)$ (i.e. $\boldsymbol{\eta}\left(\Gamma \backslash \mathrm{AC}^{2}\left([0,1] ; \mathbb{R}^{d}\right)\right)=0$ ) and

$$
\int_{\Gamma} E_{2}(\gamma) \mathrm{d} \boldsymbol{\eta}(\gamma)<\infty
$$

then $\mu \in \mathrm{AC}_{2}\left([0,1] ; \mathcal{P}_{2}\left(\mathbb{R}^{d}\right)\right)$ and it solves the continuity equation

$$
\partial_{t} \mu_{t}+\nabla \cdot(\mu \boldsymbol{v})=0
$$

for the vector field $\boldsymbol{v}$ which is barycenter of the measure $\tilde{\nu}=\mathrm{d}_{\sharp}(\boldsymbol{\eta})$ w.r.t. $\tilde{\mu}$. In fact, $\tilde{\nu}$ can be disintegrated w.r.t. its marginal $\tilde{\mu}$ by $\tilde{\nu}=\int_{Q} \nu_{t, x} \mathrm{~d} \tilde{\mu}(t, x)$ and

$$
\boldsymbol{v}(t, x)=\int_{\mathbb{R}^{d}} v \mathrm{~d} \nu_{t, x}(v)
$$

Since

$$
\int_{\Gamma} E_{2}(\gamma) \mathrm{d} \boldsymbol{\eta}(\gamma)=\int_{\Gamma} \int_{0}^{1}|\dot{\gamma}(t)|^{2} \mathrm{~d} t \mathrm{~d} \boldsymbol{\eta}(\gamma)=\int_{(0,1) \times \mathbb{R}^{d} \times \mathbb{R}^{d}}|v|^{2} \mathrm{~d} \tilde{\nu}(t, x, v)=\int_{Q}\left(\int_{\mathbb{R}^{d}}|v|^{2} \mathrm{~d} \nu_{t, x}\right) \mathrm{d} \tilde{\mu}(t, x)
$$


one immediately has by Jensen's inequality that

$$
|\boldsymbol{v}(t, x)|^{2} \leq \int_{\mathbb{R}^{d}}|v|^{2} \mathrm{~d} \nu_{t, x}(v), \quad \int_{Q}|\boldsymbol{v}|^{2} \mathrm{~d} \tilde{\mu} \leq \int_{Q}\left(\int_{\mathbb{R}^{d}}|v|^{2} \mathrm{~d} \nu_{t, x}\right) \mathrm{d} \tilde{\mu}(t, x) \leq \int_{\Gamma} E_{2}(\gamma) \mathrm{d} \boldsymbol{\eta}(\gamma) .
$$

and similarly, for convex Lagrangians,

$$
\int_{Q} L(x, \boldsymbol{v}) \mathrm{d} \tilde{\mu} \leq \int_{Q}\left(\int_{\mathbb{R}^{d}} L(x, v) \mathrm{d} \nu_{t, x}\right) \mathrm{d} \tilde{\mu}(t, x) \leq \int_{\Gamma} L(\gamma(t), \dot{\gamma}(t)) \mathrm{d} \boldsymbol{\eta}(\gamma) .
$$

The following result [3, Section 8.2] shows that any solution to the continuity equation (7.7) admits the representation (7.5) for a measure $\boldsymbol{\eta}$ tighten to $\mu$ so that (7.10) and (7.11) become in fact an identity.

Theorem 7.1 (Superposition principle) If $\mu \in \mathrm{AC}^{2}\left([0,1] ; \mathcal{P}_{2}\left(\mathbb{R}^{d}\right)\right)$ is a solution to the continuity equation (3.12) with respect to $\boldsymbol{v} \in L^{2}\left(Q, \tilde{\mu} ; \mathbb{R}^{d}\right)$, then there exists a measure $\boldsymbol{\eta} \in \mathcal{P}(\Gamma)$ concentrated on the set of curves $\gamma \in \mathrm{AC}^{2}\left([0,1] ; \mathbb{R}^{d}\right)$ which are integral solutions to the ODE's

$$
\dot{\gamma}(t)=\boldsymbol{v}(t, \gamma(t)) \quad \mathscr{L}^{1} \text {-a.e. in }(0,1),
$$

such that $\left(\mathrm{e}_{t}\right)_{\sharp} \boldsymbol{\eta}=\mu_{t}$ for every $t \in[0,1]$ and $\mathrm{e}_{\sharp} \boldsymbol{\eta}=\tilde{\mu}$.

In the following we call $\mathcal{P}_{2, p}(\Gamma)$ the collection of measures $\boldsymbol{\eta} \in \mathcal{P}(\Gamma)$ such that

$$
\int_{\Gamma} \mathrm{E}_{2}(\gamma) \mathrm{d} \boldsymbol{\eta}<\infty, \quad \mathbf{e}_{\sharp} \boldsymbol{\eta}=m \lambda \quad \text { for } m \in L^{p}(Q),
$$

and we say that $\boldsymbol{\eta}$ is tightened to $(m, \boldsymbol{v}) \in \mathrm{CE}_{2, p}(Q)$ if $\boldsymbol{\eta}$ satisfies the conditions of Theorem 7.1 . We want to study the variational properties of dynamic plans $\boldsymbol{\eta}$ tightened to minimizers of Problem 6.1.

\subsection{Lifting functions to $\Gamma$ and regularization of dynamic plans}

Let $w \in L^{0}(Q)$ and $\mathrm{w}$ be a Borel representative of $w$. If $\boldsymbol{\eta} \in \mathcal{P}_{2, p}(\Gamma)$ the measure $\tilde{\mu}=\mathrm{e}_{\sharp} \boldsymbol{\eta}$ is absolutely continuous w.r.t. $\mathscr{L}^{d+1}$ so that the function

$$
w_{\Gamma}=\mathrm{w} \circ \mathrm{e}:(0,1) \times \Gamma \rightarrow \mathbb{R}, \quad w_{\Gamma}(t, \gamma):=\mathrm{w}(t, \gamma(t))
$$

is well defined and its equivalence class modulo $\tilde{\boldsymbol{\eta}}$-negligible sets in $I \times \Gamma$ does not depend on the particular $\lambda$-representative of $w$. Similarly, we can consider the function $\ell(t, x, y):=L(x, y)$

$$
L_{\Gamma}:=\ell \circ \mathrm{d}:(0,1) \times \Gamma \rightarrow \mathbb{R}, \quad L_{\Gamma}(t, \gamma):=L(\gamma(t), \dot{\gamma}(t))
$$

Lemma 7.2 Let $u$ be a weak subsolution to $\mathrm{HJ}(H, \alpha)$ with (right continuous) representative $\mathrm{u}$ and $\alpha \in L^{q}(Q)$ and let $\boldsymbol{\eta} \in \mathcal{P}_{2, p}(\Gamma)$ be a given plan, with $\mu_{t}:=\left(\mathrm{e}_{t}\right)_{\#} \eta$. If

$$
B_{\Gamma}(t, \gamma):=\int_{0}^{t}(L(\gamma(r), \dot{\gamma}(r))+\alpha(r, \gamma(r))) \mathrm{d} r=\int_{0}^{t}\left(L_{\Gamma}(r, \gamma)+\alpha_{\Gamma}(r, \gamma)\right) \mathrm{d} r
$$

we can find a $\boldsymbol{\eta}$-negligible set $N \subset \Gamma$ and a Borel representative $u_{\star}$ of $u_{\Gamma}$ such that for every $0 \leq s \leq t \leq 1$

$$
u_{\star}(s, \gamma)+B_{\Gamma}(s, \gamma) \leq u_{\star}(t, \gamma)+B_{\Gamma}(t, \gamma) \quad \text { for every } \gamma \in \Gamma \backslash N .
$$

Morever, there exists a countable set $J$ such that for every $t \in D_{p}[\mu] \backslash J$

$$
u_{\star}(t, \gamma)=u_{\Gamma}(t, \gamma)=\mathrm{u}(t, \gamma(t)) \quad \text { for } \boldsymbol{\eta} \text {-a.e. } \gamma \in \Gamma
$$

and (7.17) also holds at $s=0$ and $t=1$ with the traces of $\mathrm{u}$ :

$$
u_{0}^{+}(\gamma(0))-u_{1}^{-}(\gamma(1)) \leq \int_{0}^{1}(L(\gamma(r), \dot{\gamma}(r))+\alpha(\gamma(r), \dot{\gamma}(r))) \mathrm{d} r=B_{\Gamma}(1, \gamma) \quad \text { for } \boldsymbol{\eta} \text {-a.e. } \gamma \text {. }
$$

Proof. Let us choose a nonnegative $\varphi \in C_{b}(\Gamma)$ such that $M(\varphi):=\int_{\Gamma} \varphi \mathrm{d} \boldsymbol{\eta}>0$. The corresponding measure $\boldsymbol{\eta}^{\varphi}:=M(\varphi)^{-1} \varphi \boldsymbol{\eta}$ induces a curve $\mu^{\varphi}$ with density $m^{\varphi} \in L^{p}(Q)$ and a vector field $\boldsymbol{v}^{\varphi}$ as in (7.5) and (7.8) such that $\left(m^{\varphi}, \boldsymbol{v}^{\varphi}\right) \in \mathrm{CE}_{2, p}(Q)$. From (5.41) of Theorem 5.12 and Fenchel inequality we get for every $s, t \in D_{p}[\mu]$ (with the obvious modifications for $s=0$ and $t=1$ )

$$
\begin{aligned}
\int_{\Gamma} \mathrm{u}(s, \gamma(s)) \mathrm{d} \boldsymbol{\eta}^{\varphi}=\int_{\mathbb{R}^{d}} \mathrm{u}_{s} \mathrm{~d} \mu_{s}^{\varphi} & \leq \int_{\mathbb{R}^{d}} \mathrm{u}_{t}^{\varphi} \mathrm{d} \mu_{t}^{\varphi}+\int_{s}^{t} \int_{\mathbb{R}^{d}}\left[L\left(x, \boldsymbol{v}^{\varphi}\right)+\alpha(r, x)\right] \mathrm{d} \tilde{\mu}^{\varphi} \\
& \leq \int_{\Gamma} \mathrm{u}(t, \gamma(t)) \mathrm{d} \boldsymbol{\eta}^{\varphi}+\int_{\Gamma} \int_{s}^{t}[L(\gamma(r), \dot{\gamma}(r))+\alpha(r, \gamma(r))] \mathrm{d} r \mathrm{~d} \boldsymbol{\eta}^{\varphi}(\gamma)
\end{aligned}
$$


and therefore

$$
\int_{\Gamma}\left(\mathrm{u}_{\Gamma}(s, \gamma)+B_{\Gamma}(s, \gamma)\right) \varphi(\gamma) \mathrm{d} \boldsymbol{\eta} \leq \int_{\Gamma}\left(\mathrm{u}_{\Gamma}(t, \gamma)+B_{\Gamma}(t, \gamma)\right) \varphi(\gamma) \mathrm{d} \boldsymbol{\eta}
$$

Since $\varphi$ is arbitrary we deduce that

$$
\mathrm{u}_{\Gamma}(s, \cdot)+B_{\Gamma}(s, \cdot) \leq \mathrm{u}_{\Gamma}(t, \cdot)+B_{\Gamma}(t, \cdot) \quad \boldsymbol{\eta} \text {-a.e. in } \Gamma .
$$

The implication $(i i) \Rightarrow($ iii $)$ of Lemma 4.3 yields (7.17); property (I.2) of the same Lemma yields (7.18) and (7.19).

We want now to extend the previous Lemma to arbitrary dynamic plans in $\mathcal{P}(\Gamma)$ concentrated on $\mathrm{AC}^{2}\left([0,1] ; \mathbb{R}^{d}\right)$ with initial and final marginals $\mu_{i}=\left(\mathrm{e}_{i}\right)_{\sharp} \boldsymbol{\eta} \ll \mathscr{L}^{d}$. We will slightly reinforce the structural assumptions 2.1 by supposing that

$$
f, L \text { are nonnegative. } L \text { is continuous in } \mathbb{R}^{d} \times \mathbb{R}^{d} .
$$

Following the approach of [2] (see also [13]), for every Borel function $\alpha \in L^{q}(Q)$ we introduce a precise representative of $\alpha$, obtained by convolving $\alpha$ with the (symmetric) Heat kernel $g_{\varepsilon}(3.29)$. To simplify some aspects concerning measurability, we use here a definition based on a given sequence $(\varepsilon(n))_{n \in \mathbb{N}} \subset(0,1]$ such that $\varepsilon(n) \downarrow 0$ as $n \rightarrow \infty$ (we may choose, e.g. $\varepsilon(n):=2^{-n}$ ).

$$
\hat{\alpha}(t, x):=\limsup _{n \rightarrow \infty} \alpha_{n}(t, x), \quad \alpha_{n}(t, x):=\left(\alpha(t, \cdot) * g_{\varepsilon(n)}\right)(x)=\int_{\mathbb{R}^{d}} \alpha(x+\varepsilon(n) y) g_{1}(y) \mathrm{d} y .
$$

Notice that $\alpha=\hat{\alpha} \lambda$-a.e. in $\mathbb{R}^{d}$; moreover, if $\alpha^{\prime}=\alpha \lambda$-a.e. in $Q$, then there exists a $\mathscr{L}^{1}$-negligible set $N \subset(0,1)$ such that $\hat{\alpha}^{\prime}(t, x)=\hat{\alpha}(t, x)$ for every $t \in(0,1) \backslash N$ and $x \in \mathbb{R}^{d}$. In particular, the function $\hat{\alpha}_{\Gamma}=\hat{\alpha} \circ \mathrm{e}$ is well defined for every $\boldsymbol{\eta} \in \mathcal{P}_{2}(\Gamma)$, since the first marginal of $\mathrm{e}_{\sharp} \boldsymbol{\eta}$ is $\mathscr{L}^{1}$, and $\hat{\alpha}^{\prime}(\cdot, \gamma(\cdot))=\hat{\alpha}(\cdot, \gamma(\cdot)) \mathscr{L}^{1}$-a.e. in $(0,1)$ for $\boldsymbol{\eta}$-a.e. $\gamma$. Notice that the composition (7.14) for arbitrary $\alpha \in L^{q}(Q)$ is well defined only for $\boldsymbol{\eta} \in \mathcal{P}_{2, p}(\Gamma)$ : the choice of the precise representative $\hat{\alpha}$ extends this operation to arbitrary $\boldsymbol{\eta} \in \mathcal{P}_{2}(\Gamma)$.

We will also consider the corresponding maximal function (depending on the sequence $\varepsilon(n)$ )

$$
\hat{M} \alpha(t, x):=\sup _{n \in \mathbb{N}} \int_{\mathbb{R}^{d}}|\alpha(x+\varepsilon(n) y)| g_{1}(y) \mathrm{d} y=\sup _{n \in \mathbb{N}} \int_{\mathbb{R}^{d}}|\alpha(z)| g_{\varepsilon(n)}(x-z) \mathrm{d} z .
$$

Since clearly $\hat{M} \alpha$ is bounded by the maximal function $M \alpha$, i.e.

$$
\hat{M} \alpha(t, x) \leq M \alpha(t, x):=\sup _{r>0} \int_{\mathbb{R}^{d}}|\alpha(x+r y)| g_{1}(y) \mathrm{d} y,
$$

as noticed in [2, p. 456], we have

$$
\hat{M} \alpha(t, x)=\hat{M} \hat{\alpha}(t, x), \quad \alpha_{n}(t, x) \leq \hat{M} \alpha(t, x) \text { if } n \in \mathbb{N}, \quad \hat{M} \alpha \in L^{q}(Q) .
$$

Let us fix a few measurability properties of $\hat{\alpha}$ and $\hat{M} \alpha$ which will turn to be useful in the following. Recall that a Borel function $g: Q \rightarrow[0,+\infty]$ is a normal integrand if it is lower semicontinuous w.r.t. $x$ for $\mathscr{L}^{1}$-a.e. $t \in(0,1)$ (see [16, Chap. VIII, Def. 1.1]).

Lemma 7.3 Let $\alpha: Q \rightarrow \mathbb{R}$ be a Borel map in $L^{q}(Q)$ and let $\hat{\alpha}, \hat{M} \alpha$ be defined as in (7.24) and (7.25).

1. $\hat{M} \alpha$ is a Borel and normal positive integrand so that

$$
\begin{gathered}
\text { the functional } \quad \gamma \mapsto \mathcal{M}_{\alpha}[\gamma]:=\int_{0}^{1} \hat{M} \alpha(t, \gamma(t)) \mathrm{d} t \quad \text { is lower semicontinuous in } \Gamma \text {, } \\
\mathrm{D}\left(\mathcal{M}_{\alpha}\right):=\left\{\gamma \in \Gamma: \mathcal{M}_{\alpha}[\gamma]<\infty\right\} \quad \text { is a } F_{\sigma} \text {, thus Borel, subset of } \Gamma \text {. }
\end{gathered}
$$

2. $\hat{\alpha}$ is a Borel map with values in $[0,+\infty]$, the composition $t \mapsto \hat{\alpha}(t, \gamma(t))$ is a Borel curve for every $\gamma \in \Gamma$, and the map $\hat{\alpha}_{\Gamma}: \gamma \rightarrow \hat{\alpha}(\cdot, \gamma(\cdot))$ is Borel from $\Gamma$ to $L^{0}((0,1) ;[0,+\infty])$.

3. The integral functional $\mathcal{I}_{\alpha}: \Gamma \rightarrow[0,+\infty]$

$$
\mathcal{I}_{\alpha}[\gamma]:=\int_{0}^{1} \hat{\alpha}(t, \gamma(t)) \mathrm{d} t
$$

is a Borel map from $\Gamma$ to $[0,+\infty]$. 
Proof. Since we have chosen a Borel representative $\alpha: Q \rightarrow \mathbb{R}$, for every $n \in \mathbb{N}$ the functions $\alpha_{n}$ : $Q \rightarrow[0,+\infty]$ are Borel and also Caratheodory integrands, since they are finite and continuous w.r.t. $x$ for a.e. $t \in(0,1)$; moreover they satisfy

$$
\sup _{x \in \mathbb{R}^{n}}\left|\alpha_{n}(t, x)\right| \leq c_{n}\left(\int_{\mathbb{R}^{d}}|\alpha(t, x)|^{q}\right)^{1 / q}, \quad \int_{0}^{1}\left(\sup _{x \in \mathbb{R}^{n}}\left|\alpha_{n}(t, x)\right|\right)^{q} \mathrm{~d} t \leq c_{n}\|\alpha\|_{L^{q}(Q)}^{q}
$$

for some constant $c_{n}>0$. It follows that for every $\gamma \in \Gamma$ the curve $t \mapsto \alpha_{n}(t, \gamma(t))$ is Borel and the map $\gamma \mapsto \alpha_{n}(\cdot, \gamma(\cdot))$ is continuous from $\Gamma$ to $L^{q}(0,1)$ and a fortiori to $L^{0}(0,1)$.

We deduce that $\hat{M} \alpha$ is Borel and normal, so that (7.28) follows by [16, Chap. VIII, Prop. 1.4].

Concerning the second point, it easily follows since setting $\alpha_{n, m}:=\sup _{m \leq k \leq n} \alpha_{k}, \hat{\alpha}_{m}:=\lim _{n \rightarrow \infty} \alpha_{m, n}$, we have $\hat{\alpha}(t, x):=\lim _{m \rightarrow \infty} \hat{\alpha}_{m}$ which is therefore pointwise limit of Borel maps. The same structure holds for the corresponding composition maps from the (complete and separable metric space) $\Gamma$ to the (complete and separable metric space) $L^{0}((0,1) ;[0,+\infty])$, so that the map $\hat{\alpha}_{\Gamma}: \gamma \rightarrow \hat{\alpha}(\cdot, \gamma(\cdot))$ is Borel from $\Gamma$ to $L^{0}((0,1) ;[0,+\infty])$.

It follows that the integral functional $\mathcal{I}_{\alpha}$ is a Borel map from $\Gamma$ to $[0,+\infty]$, being the composition of a lower semicontinuous map (the integral, from $\left.L^{0}((0,1) ;[0,+\infty])\right)$ to $\mathbb{R}$ ) with a Borel map.

If $\boldsymbol{\eta} \in \mathcal{P}_{2, p}(\Gamma)$ then the function $(\hat{M} \alpha)$ o e belongs to $L^{1}(I \times \Gamma, \tilde{\boldsymbol{\eta}})$. Since also $\hat{M} \alpha(t, \cdot)$ is everywhere defined in $\mathbb{R}^{d}$ for $\mathscr{L}^{1}$-a.e. $t \in(0,1)$, we will more generally consider dynamic plans $\boldsymbol{\eta} \in \mathcal{P}_{2}(\Gamma)$ concentrated on $\mathrm{D}\left(\mathcal{M}_{\alpha}\right)$, so that $\int_{0}^{1}(\hat{M} \alpha)(t, \gamma(t)) \mathrm{d} t=\int_{0}^{1}(\hat{M} \alpha)_{\Gamma}(t, \gamma) \mathrm{d} t<\infty$ for $\boldsymbol{\eta}$-a.e. $\gamma$.

Let us introduce the sets

$$
\begin{aligned}
\Gamma_{\alpha, k} & :=\left\{\gamma \in \operatorname{AC}^{2}\left([0,1] ; \mathbb{R}^{d}\right): \mathrm{E}_{2}[\gamma]+\mathcal{M}_{\alpha}[\gamma] \leq k\right\}, \quad k \in \mathbb{N} \\
\Gamma_{\alpha} & :=\left\{\gamma \in \operatorname{AC}^{2}\left([0,1] ; \mathbb{R}^{d}\right): \mathcal{M}_{\alpha}[\gamma]<+\infty\right\}=\bigcup_{k \in \mathbb{N}} \Gamma_{\alpha, k} .
\end{aligned}
$$

Lemma 7.4 (Trace inequality along plans in $\mathcal{P}_{2}(\Gamma)$ ) Let $u$ be a weak subsolution to $\mathrm{HJ}(H, \alpha)$ with nonnegative $\alpha \in L^{q}(Q)$ and traces $u_{0}^{+}, u_{1}^{-} \in L^{0}\left(\mathbb{R}^{d}, \overline{\mathbb{R}}\right)$ and let $\boldsymbol{\eta} \in \mathcal{P}(\Gamma)$ be a dynamic plan satisfying

$$
\mu_{i}=\left(\mathrm{e}_{i}\right)_{\sharp} \boldsymbol{\eta} \ll \mathscr{L}^{d}, i=0,1 ; \quad \gamma \in \Gamma_{\alpha} \quad \text { for } \boldsymbol{\eta} \text {-a.e. } \gamma \text {. }
$$

Then (7.19) holds.

Proof. Let us consider two Borel representatives of $u_{0}^{+}, u_{1}^{-}$and set $U_{0 k}:=\left\{x \in \mathbb{R}^{d}:-k \leq u_{0}^{+}(x) \leq k\right\}$ and $U_{1 k}:=\left\{x \in \mathbb{R}^{d}:-k \leq u_{1}^{-}(x) \leq k\right\} ;$ since $u_{0}^{+}$takes values in $\mathbb{R} \cup\{-\infty\}$ and $u_{1}^{-}$takes values in $\mathbb{R} \cup\{+\infty\}$, we have

$$
U_{0}:=\bigcup_{k \in \mathbb{N}} U_{0 k}=\left\{x \in \mathbb{R}^{d}: u_{0}^{+}>-\infty\right\}, \quad U_{1}:=\bigcup_{k \in \mathbb{N}} U_{1 k}=\left\{x \in \mathbb{R}^{d}: u_{1}^{-}<+\infty\right\} .
$$

We introduce the truncations $T_{k}(r):=-k \vee r \wedge k$ and the corresponding maps $u_{0 k}:=T_{k}\left(u_{0}^{+}\right)$and $u_{1 k}:=$ $T_{k}\left(u_{1}^{-}\right)$. We set

$$
U_{i k}^{r}:=\left\{x \in U_{k}: \lim _{n \rightarrow \infty} \int_{\mathbb{R}^{d}}\left|u_{i k}(x+\varepsilon(n) y)-u_{i}(x)\right| g_{1}(y) \mathrm{d} y=0\right\} .
$$

Since $u_{i k} \in L^{\infty}\left(\mathbb{R}^{d}\right)$ we know that $\lim _{n \rightarrow \infty} \int_{\mathbb{R}^{d}}\left|u_{i k}(x+\varepsilon(n) y)-u_{i k}(x)\right| g_{1}(y) \mathrm{d} y=0$ for $\mathscr{L}^{d}$-a.e. point of $\mathbb{R}^{d}$, so that $\mathscr{L}^{d}\left(U_{i k} \backslash U_{i k}^{r}\right)=0$ for every $k \in \mathbb{R}^{d}$. We eventually set $U_{i}^{r}:=\bigcup_{k \in \mathbb{N}} U_{i k}^{r}$ and notice that

$$
\boldsymbol{\eta}\left(\left\{\gamma \in \Gamma: \gamma(i) \in U_{i} \backslash U_{i}^{r}, i=0,1\right\}\right)=0
$$

since $\mu_{i} \ll \mathscr{L}^{d}$. For every $k \in \mathbb{N}$ let us introduce the sets

$$
\Sigma_{k}:=\left\{\gamma \in \Gamma_{\alpha, k}: \gamma(i) \in U_{i k}^{r}, i=0,1\right\}
$$

Since (7.19) is trivially satisfied if $\gamma(0) \in \mathbb{R}^{d} \backslash U_{0}$ or $\gamma(1) \in \mathbb{R}^{d} \backslash U_{1}$, it is sufficient to prove (7.19) in the case when $\boldsymbol{\eta}$ is concentrated in the set $\Sigma_{k}$. We consider the cartesian product $X:=\Gamma \times \mathbb{R}^{d}$ endowed with the Probability measure $\boldsymbol{\sigma}:=\boldsymbol{\eta} \otimes\left(g_{1} \mathscr{L}^{d}\right)$ and the Borel map $R_{n}: \Gamma \times \mathbb{R}^{d} \rightarrow \Gamma, R_{n}(\gamma, y):=\gamma+\varepsilon(n) y$. Setting $\boldsymbol{\eta}_{n}:=\left(R_{n}\right)_{\sharp} \boldsymbol{\sigma}$ it is easy to check that $\boldsymbol{\eta}_{n}$ is concentrated on $\mathrm{AC}^{2}\left([0,1] ; \mathbb{R}^{d}\right)$ with

$$
\int \mathrm{E}_{2}[\gamma] \mathrm{d} \boldsymbol{\eta}_{n}=\int \mathrm{E}_{2}\left[R_{n}(\gamma, y)\right] \mathrm{d} \boldsymbol{\sigma}(\gamma, y)=\int \mathrm{E}_{2}[\gamma] \mathrm{d} \boldsymbol{\sigma}(\gamma, y)=\int \mathrm{E}_{2}[\gamma] \mathrm{d} \boldsymbol{\eta}(\gamma)
$$


since translations by vectors $\varepsilon(n) y$ do not modify the energy of a curve. On the other hand, setting $\mu_{t}:=$ $\left(\mathrm{e}_{t}\right)_{\sharp} \boldsymbol{\eta}$, we have

$$
\left(\mathrm{e}_{t}\right)_{\sharp} \boldsymbol{\eta}_{n}=\left(\mu_{t}\right) * g_{\varepsilon(n)} \quad \text { for every } t \in[0,1],
$$

since for every $\phi \in C_{b}\left(\mathbb{R}^{d}\right)$

$$
\begin{aligned}
\int_{\mathbb{R}^{d}} \phi(x) \mathrm{d}\left(\mathrm{e}_{t}\right)_{\sharp} \boldsymbol{\eta}_{n} & =\int_{\Gamma} \phi(\gamma(t)) \mathrm{d} \boldsymbol{\eta}_{n}(\gamma)=\int_{\Gamma \times \mathbb{R}^{d}} \phi(\gamma(t)+\varepsilon(n) y) g_{1}(y) \mathrm{d} \boldsymbol{\eta}(\gamma) \mathrm{d} y \\
& =\int_{\mathbb{R}^{d}}\left(\int_{\mathbb{R}^{d}} \phi(x+\varepsilon(n) y) \mathrm{d} \mu_{t}(x)\right) g_{1}(y) \mathrm{d} y=\int_{\mathbb{R}^{d}} \phi \mathrm{d}\left(\mu_{t} * g_{\varepsilon(n)}\right) .
\end{aligned}
$$

(3.30) shows that $\boldsymbol{\eta}_{n} \in \mathcal{P}_{2, p}\left(\mathbb{R}^{d}\right)$. Applying (17.19) to the truncated subsolutions $u_{k}:=T_{k}(u)$ and observing that $u_{k}$ is a subsolution to $-\partial_{t} u_{k}+H\left(x, D u_{k}\right) \leq \alpha$,

$$
\begin{aligned}
T_{k}\left(u_{0}^{+}(\gamma(0)+\varepsilon(n) y)\right) & -T_{k}\left(u_{1}^{-}(\gamma(1)+\varepsilon(n) y)\right) \\
& \leq \int_{0}^{1}(L(\gamma(r)+\varepsilon(n) y, \dot{\gamma}(r))+\alpha(r, \gamma(r)+\varepsilon(n) y)) \mathrm{d} r \quad \text { for } \boldsymbol{\sigma} \text {-a.e. }(\gamma, y),
\end{aligned}
$$

A further integration w.r.t. $y$ yields that there exists a Borel set $\Sigma_{\star} \subset \Sigma_{k}$ such that $\boldsymbol{\eta}\left(\Sigma_{k} \backslash \Sigma_{\star}\right)=0$ and

$$
\begin{aligned}
\int_{\mathbb{R}^{d}} T_{k}\left(u_{0}^{+}(\gamma(0)+\varepsilon(n) y)\right) & g_{1}(y) \mathrm{d} y-\int_{\mathbb{R}^{d}} T_{k}\left(u_{1}^{-}(\gamma(1)+\varepsilon(n) y)\right) g_{1}(y) \mathrm{d} y \\
& \leq \int_{\mathbb{R}^{d}}\left(\int_{0}^{1}(L(\gamma(r)+\varepsilon(n) y, \dot{\gamma}(r))+\alpha(r, \gamma(r)+\varepsilon(n) y)) \mathrm{d} r\right) g_{1}(y) \mathrm{d} y
\end{aligned}
$$

for every $n \in \mathbb{N}$ and $\gamma \in \Sigma_{\star}$. We can then pass to the limit as $n \rightarrow \infty$ : the structural bounds (2.20) (with $\left.c_{H}^{+}=0\right)$ yield

$$
0 \leq \int_{0}^{1} L(\gamma(r)+\varepsilon(n) y, \dot{\gamma}(r)) \mathrm{d} r \leq c_{H}^{-}\left(1+2|y|^{2}\right)+2 c_{H}^{-} \int_{0}^{1}|\gamma(r)|^{2} \mathrm{~d} r+\frac{c_{H}}{2} \mathrm{E}_{2}[\gamma]
$$

so that Lebesgue Dominated Convergence theorem and the continuity of $L$ yield

$$
\lim _{n \rightarrow \infty} \int_{\mathbb{R}^{d}}\left(\int_{0}^{1} L(\gamma(r)+\varepsilon(n) y, \dot{\gamma}(r)) \mathrm{d} r\right) g_{1}(y) \mathrm{d} y=\int_{0}^{1} L(\gamma(r), \dot{\gamma}(r)) \mathrm{d} r .
$$

Similarly we get

$$
\int_{\mathbb{R}^{d}}\left(\int_{0}^{1} \alpha(r, \gamma(r)+\varepsilon(n) y) \mathrm{d} r\right) g_{1}(y) \mathrm{d} y=\int_{0}^{1} \alpha_{n}(r, \gamma(r)) \mathrm{d} r, \quad \alpha_{n}:=\alpha(t, \cdot) * g_{\varepsilon(n)} ;
$$

since $0 \leq \alpha_{n} \leq \hat{M} \alpha$ and $\int_{0}^{1} \hat{M} \alpha(t, \gamma(t)) \mathrm{d} t \leq k$ by assumption, we conclude that

$$
\limsup _{n \rightarrow \infty} \int_{\mathbb{R}^{d}}\left(\int_{0}^{1} \alpha(r, \gamma(r)+\varepsilon(n) y) \mathrm{d} r\right) g_{1}(y) \mathrm{d} y \leq \int_{0}^{1} \hat{\alpha}(r, \gamma(r)) \mathrm{d} r .
$$

Combining (7.40) and (7.42) and the fact that $\gamma(i) \in U_{i k}^{r}$ we eventually get

$$
u_{0}^{+}(\gamma(0))-u_{1}^{-}(\gamma(1)) \leq \int_{0}^{1}(L(\gamma(r), \dot{\gamma}(r))+\hat{\alpha}(r, \gamma(r))) \mathrm{d} r \quad \text { for every } \gamma \in \Sigma_{\star} .
$$

\subsection{Modified Lagrangian cost and optimal dynamic plans}

We can now introduce a cost function associated to the modified Lagrangian (6.59) for any nonnegative Borel map $\alpha \in L^{q}(Q)$. Since we want to compute $\alpha$ along dynamic plans $\boldsymbol{\eta}^{\prime} \in \mathcal{P}(\Gamma)$ which may not have absolutely continuous marginals $\mu_{t}^{\prime}=\left(\mathrm{e}_{t}\right)_{\sharp} \boldsymbol{\eta}^{\prime}$, we will add an extra summability condition in terms of the maximal function $\hat{M} \alpha$, as in [2], and we replace $L_{\alpha}$ by

$$
L_{\hat{\alpha}}(t, x, \boldsymbol{v}):=L(x, \boldsymbol{v})+\hat{\alpha}(t, x) \quad \text { with } \quad \mathrm{L}_{\hat{\alpha}}[\gamma]:=\int_{0}^{1} L_{\hat{\alpha}}(t, \gamma(t), \dot{\gamma}(t)) \mathrm{d} t \quad \text { for every } \gamma \in \Gamma_{\alpha},
$$

where $\Gamma_{\alpha}$ is defined by (7.32). 
Definition 7.5 (Modified Lagrangian cost) For every nonnegative Borel $\alpha \in L^{q}(Q)$ let $\hat{\alpha}, \hat{M} \alpha$, and $\Gamma_{\alpha}$ be defined as in (7.24), (7.25) and (7.32). We consider the set

$$
X_{\alpha}:=\left(\mathrm{e}_{0}, \mathrm{e}_{1}\right)\left(\Gamma_{\alpha}\right)=\left\{(\gamma(0), \gamma(1)): \gamma \in \Gamma_{\alpha}\right\}
$$

The Lagrangian cost $c_{\alpha}: \mathbb{R}^{d} \times \mathbb{R}^{d} \rightarrow[0,+\infty]$ is defined by

$$
c_{\hat{\alpha}}\left(x_{0}, x_{1}\right):=\inf \left\{\int_{0}^{1} L_{\hat{\alpha}}(t, \gamma(t), \dot{\gamma}(t)) \mathrm{d} t: \gamma \in \Gamma_{\alpha}, \gamma(0)=x_{0}, \gamma(1)=x_{1}\right\} \quad \text { if }\left(x_{0}, x_{1}\right) \in X_{\alpha},
$$

with the usual convention to set $c_{\hat{\alpha}}\left(x_{0}, x_{1}\right):=+\infty$ if $\left(x_{0}, x_{1}\right) \notin X_{\alpha}$.

Let us preliminary study the measurability properties of $c_{\hat{\alpha}}$.

Lemma 7.6 $X_{\alpha}$ is a $F_{\sigma}$ set in $\mathbb{R}^{d} \times \mathbb{R}^{d}$. For every $\boldsymbol{\mu} \in \mathcal{P}\left(\mathbb{R}^{d} \times \mathbb{R}^{d}\right) c_{\hat{\alpha}}$ is $\boldsymbol{\mu}$-measurable (equivalently, $c_{\hat{\alpha}}$ is universally measurable). Moreover, for every $\varepsilon>0$ there exists a $\boldsymbol{\mu}$-measurable map $\boldsymbol{\omega}: X_{\hat{\alpha}} \rightarrow \Gamma$ such that for every $\left(x_{0}, x_{1}\right) \in X_{\hat{\alpha}}$ the curve $\gamma=\boldsymbol{\omega}\left(x_{0}, x_{1}\right)$ satisfies

$$
\gamma \in \Gamma_{\hat{\alpha}}, \quad \gamma(i)=x_{i}, \quad \int_{0}^{1} L_{\hat{\alpha}}(t, \gamma(t), \dot{\gamma}(t)) \mathrm{d} t \leq c_{\hat{\alpha}}\left(x_{0}, x_{1}\right)+\varepsilon .
$$

Proof. We can write

$$
X_{\alpha}=\bigcup_{k \in \mathbb{N}}\left\{\left(x_{0}, x_{1}\right) \in \mathbb{R}^{d} \times \mathbb{R}^{d}:\left|x_{0}\right|+\left|x_{1}\right| \leq k, \exists \gamma \in \Gamma_{\alpha, k}: \gamma(i)=x_{i}, i=0,1\right\},
$$

and it is easy to check that each set contributing to the countable union in (7.48) is compact.

By Lemma $7.3 \Gamma_{\alpha}$ is a $F_{\sigma}$ subset of $\Gamma$ and the map $\mathrm{L}_{\hat{\alpha}}: \Gamma_{\alpha} \rightarrow[0,+\infty]$ is Borel, so that for every $r \in(0,+\infty]$ the set $\Xi_{\hat{\alpha}}(r):=\left\{\gamma \in \Gamma_{\alpha}: \mathrm{L}_{\hat{\alpha}}[\gamma]<r\right\}$ is Borel in $\Gamma$.

Since the sublevel $\left\{\left(x_{0}, x_{1}\right) \in \mathbb{R}^{d} \times \mathbb{R}^{d}: c_{\hat{\alpha}}\left(x_{0}, x_{1}\right)<r\right\}$ coincides with the image of $\Xi_{\hat{\alpha}}(r)$ through the continuous map $\left(\mathrm{e}_{0}, \mathrm{e}_{1}\right): \Gamma \rightarrow \mathbb{R}^{d} \times \mathbb{R}^{d}$, the Projection Theorem (see, e.g. , [14, Thm. III.23], [8, Thm. 7.4.1]) then shows that $\left\{c_{\hat{\alpha}}<r\right\}$ is a Souslin set and therefore universally measurable. $c_{\hat{\alpha}}$ is universally measurable as well.

Let us now consider the sets

$$
W^{\prime}:=\left\{\left(\left(x_{0}, x_{1}\right), \gamma\right) \in\left(\mathbb{R}^{d} \times \mathbb{R}^{d}\right) \times \Gamma: x_{0}=\gamma(0), x_{1}=\gamma(1)\right\}, \quad W^{\prime \prime}:=W \cap\left(X_{\alpha} \times \Gamma_{\alpha}\right) ;
$$

since $W^{\prime}$ is closed, $W^{\prime \prime}$ is a Borel subset of $\left(\mathbb{R}^{d} \times \mathbb{R}^{d}\right) \times \Gamma$. For every $\varepsilon>0$ we eventually set

$$
W_{\varepsilon}:=\left\{\left(\left(x_{0}, x_{1}\right), \gamma\right) \in W^{\prime \prime}: \mathrm{L}_{\hat{\alpha}}[\gamma]-c_{\hat{\alpha}}\left(x_{0}, x_{1}\right)<\varepsilon\right\}
$$

$W_{\varepsilon}$ is a $\mathscr{B}_{\boldsymbol{\mu}} \otimes \mathscr{B}(\Gamma)$-measurable subset of $\left(\mathbb{R}^{d} \times \mathbb{R}^{d}\right) \times \Gamma$, where $\mathscr{B}_{\boldsymbol{\mu}}$ is the $\sigma$-algebra of $\boldsymbol{\mu}$-measurable subsets of $\mathbb{R}^{d} \times \mathbb{R}^{d}$ and $\mathscr{B}(\Gamma)$ is the $\sigma$-algebra of Borel subsets of $\Gamma$. The projection of $W_{\varepsilon}$ on the first component coincides with $X_{\alpha}$. Applying Aumann Selection Theorem [14, Thm. III.22], 8, Thm. 6.9.13] we can find a $\mathscr{B}_{\boldsymbol{\mu}}$-measurable map $\boldsymbol{\omega}: X_{\alpha} \rightarrow \Gamma$ such that $\left(x_{0}, x_{1}, \boldsymbol{\omega}\left(x_{0}, x_{1}\right)\right) \in W_{\varepsilon}$ for every $x_{0}, x_{1} \in X_{\alpha}$.

We can now state the main result connecting solutions of MFPP and dynamic plans.

Theorem 7.7 Let us assume that the structural properties 2.1 hold together with (7.23). Let $\mu_{i}=m_{i} \mathscr{L}^{d} \in$ $\mathcal{P}_{2, p}^{r}\left(\mathbb{R}^{d}\right)$, let $\boldsymbol{\eta} \in \mathcal{P}_{2, p}(\Gamma)$ a dynamic plan satisfying $\left(\mathrm{e}_{i}\right)_{\sharp} \boldsymbol{\eta}=\mu_{i}, i=0,1$, and let us call $m \in L^{p}(Q)$ a Borel density of $\mu=\mathrm{e}_{\sharp} \boldsymbol{\eta}$ and $\alpha:=f(\cdot, m)$.

Then the following conditions are equivalent:

(i) $\boldsymbol{\eta}$ is tightened to an optimal solution $(m, \boldsymbol{v})$ of Problem 6.1.

(ii) There exist a weak subsolution $u \in L_{l o c}^{1}(Q)$ to $-\partial_{t} u+H(x, D u) \leq \alpha$ such that

$$
u_{0}^{+}(\gamma(0))-u_{1}^{-}(\gamma(1))=\int_{0}^{1} L_{\hat{\alpha}}(t, \gamma(t), \dot{\gamma}(t)) \mathrm{d} t \quad \text { for } \boldsymbol{\eta} \text {-a.e. } \gamma \text {. }
$$

(iii) We have

$$
\begin{gathered}
\int_{\Gamma}\left(\int_{0}^{1} L_{\hat{\alpha}}(t, \gamma(t), \dot{\gamma}(t)) \mathrm{d} t\right) \mathrm{d} \boldsymbol{\eta} \leq \int_{\Gamma}\left(\int_{0}^{1} L_{\hat{\alpha}}(t, \gamma(t), \dot{\gamma}(t)) \mathrm{d} t\right) \mathrm{d} \boldsymbol{\eta}^{\prime} \\
\text { if } \boldsymbol{\eta}^{\prime} \in \mathcal{P}(\Gamma),\left(\mathrm{e}_{i}\right)_{\sharp} \boldsymbol{\eta}^{\prime}=\mu_{i}, \gamma \in \mathrm{AC}^{2}\left([0,1] ; \mathbb{R}^{d}\right) \text { and } \int_{0}^{1} \hat{M} \alpha(t, \gamma(t)) \mathrm{d} t<\infty \text { for } \boldsymbol{\eta}^{\prime}-\text { a.e. } \gamma \text {. }
\end{gathered}
$$


(iv) We have

E.1 $\boldsymbol{\eta}$ is concentrated on $c_{\alpha}$-minimizing paths, i.e.

$$
\int_{0}^{1} L_{\hat{\alpha}}(t, \gamma(t), \dot{\gamma}(t)) \mathrm{d} t=c_{\alpha}(\gamma(0), \gamma(1)) \quad \text { for } \boldsymbol{\eta} \text {-a.e. } \gamma \in \Gamma \text {. }
$$

E.2 The plan $\boldsymbol{\mu}_{\boldsymbol{\eta}}:=\mathrm{e}_{\sharp} \boldsymbol{\eta}=\left(\mathrm{e}_{0}, \mathrm{e}_{1}\right)_{\sharp} \boldsymbol{\eta}$ is a solution to the optimal transport problem induced by $c_{\hat{\alpha}}$ :

$$
\int_{\mathbb{R}^{d} \times \mathbb{R}^{d}} c_{\hat{\alpha}}\left(x_{0}, x_{1}\right) \mathrm{d} \boldsymbol{\mu}_{\boldsymbol{\eta}}=\min \left\{\int_{\mathbb{R}^{d} \times \mathbb{R}^{d}} c_{\hat{\alpha}}\left(x_{0}, x_{1}\right) \mathrm{d} \boldsymbol{\mu}: \boldsymbol{\mu} \in \mathcal{P}\left(\mathbb{R}^{d} \times \mathbb{R}^{d}\right), \pi_{\sharp}^{i} \boldsymbol{\mu}=\mu_{i}\right\} .
$$

If one of the above equivalent conditions hold, then every optimal solution $(u, \alpha)$ of Problem 6.7 satisfies (7.49), and provides a pair of optimal Kantorovich potentials for the problem (7.52), i.e.

$$
u_{0}^{+}\left(x_{0}\right)-u_{1}^{-}\left(x_{1}\right) \leq c_{\hat{\alpha}}\left(x_{0}, x_{1}\right) \quad \text { in } \mathbb{R}^{d} \times \mathbb{R}^{d} ; \quad u_{0}^{+}\left(x_{0}\right)-u_{1}^{-}\left(x_{1}\right)=c_{\hat{\alpha}}\left(x_{0}, x_{1}\right) \quad \text { for } \boldsymbol{\mu}_{\boldsymbol{\eta}^{-a . e .}} x_{0}, x_{1} .
$$

Moreover $\mathrm{u}(\cdot, \gamma(\cdot)) \in W^{1,1}(0,1)$ with

$$
\begin{aligned}
\dot{\gamma}(t) & =-D_{p} H(x, D u(t, \gamma(t)) & & \text { a.e. in }(0,1), \\
\frac{\mathrm{d}}{\mathrm{d} t} \mathrm{u}(t, \gamma(t)) & =L(\gamma(t), \dot{\gamma}(t))+\alpha(t, \gamma(t)) & & \text { a.e. in }(0,1),
\end{aligned}
$$

for $\boldsymbol{\eta}$-a.e. $\gamma \in \Gamma$.

Proof. We divide the proof in various steps.

Claim 1: $(i) \Rightarrow($ ii $)$ We can select an optimal pair $(u, \alpha)$ solving Problem 6.7. Since $\boldsymbol{\eta} \in \mathcal{P}_{2, p}(\Gamma)$ (7.19) shows that

$$
u_{0}^{+}(\gamma(0))-u_{1}^{-}(\gamma(1)) \leq \int_{0}^{1} L_{\hat{\alpha}}(t, \gamma(t), \dot{\gamma}(t)) \mathrm{d} t \quad \text { for } \boldsymbol{\eta} \text {-a.e. } \gamma
$$

On the other, thanks to (6.63) we have

$$
\begin{aligned}
\int_{\Gamma}\left(u_{0}^{+}(\gamma(0))-u_{1}^{-}(\gamma(1))\right) \mathrm{d} \boldsymbol{\eta} & =\int_{\mathbb{R}^{d}} u_{0}^{+} \mathrm{d} \mu_{0}-\int_{\mathbb{R}^{d}} u_{1}^{-} \mathrm{d} \mu_{1}=\int_{Q} L_{\alpha}(t, x, \boldsymbol{v}) m \mathrm{~d} x \mathrm{~d} t \\
& =\int_{Q} L_{\hat{\alpha}}(t, x, \boldsymbol{v}) m \mathrm{~d} x \mathrm{~d} t=\int_{\Gamma}\left(\int_{0}^{1} L_{\hat{\alpha}}(t, \gamma(t), \dot{\gamma}(t)) \mathrm{d} t\right) \mathrm{d} \boldsymbol{\eta}
\end{aligned}
$$

so that (7.49) holds.

Claim 2: $(i i) \Rightarrow(i i i)$ It is sufficient to apply Lemma 7.4 and use the fact that $\left(\mathrm{e}_{i}\right)_{\sharp} \boldsymbol{\eta}^{\prime}=\mu_{i}$ :

$$
\begin{aligned}
\int_{\Gamma}\left(\int_{0}^{1} L_{\hat{\alpha}}(t, \gamma(t), \dot{\gamma}(t)) \mathrm{d} t\right) \mathrm{d} \boldsymbol{\eta} & =\int_{\Gamma}\left(u_{0}^{+}(\gamma(0))-u_{1}^{-}(\gamma(1))\right) \mathrm{d} \boldsymbol{\eta}=\int_{\mathbb{R}^{d}} u_{0}^{+} \mathrm{d} \mu_{0}-\int_{\mathbb{R}^{d}} u_{1}^{-} \mathrm{d} \mu_{1} \\
& =\int_{\Gamma}\left(u_{0}^{+}(\gamma(0))-u_{1}^{-}(\gamma(1))\right) \mathrm{d} \boldsymbol{\eta}^{\prime} \leq \int_{\Gamma}\left(\int_{0}^{1} L_{\hat{\alpha}}(t, \gamma(t), \dot{\gamma}(t)) \mathrm{d} t\right) \mathrm{d} \boldsymbol{\eta}^{\prime}
\end{aligned}
$$

Claim 3: $($ iii $) \Rightarrow($ iv $)$ Since $\hat{M} \alpha \in L^{p}(Q)$ we have $\int_{\Gamma} \int_{0}^{1} \hat{M} \alpha(t, \gamma(t)) \mathrm{d} t \mathrm{~d} \boldsymbol{\eta}(\gamma)<\infty$ so that $\boldsymbol{\eta}$ is concentrated on $\Gamma_{\alpha}$. Conditions E.1, E.2 are then equivalent to say that

$$
\int_{\Gamma} \int_{0}^{1} L_{\hat{\alpha}}(t, \gamma(t), \dot{\gamma}(t)) \mathrm{d} t \mathrm{~d} \boldsymbol{\eta}(\gamma)=\min \left\{\int_{\mathbb{R}^{d} \times \mathbb{R}^{d}} c_{\hat{\alpha}}\left(x_{0}, x_{1}\right) \mathrm{d} \boldsymbol{\mu}: \boldsymbol{\mu} \in \mathcal{P}\left(\mathbb{R}^{d} \times \mathbb{R}^{d}\right), \pi_{\sharp}^{i} \boldsymbol{\mu}=\mu_{i}\right\} .
$$

We argue by contradiction and we assume that there exists $\delta>0$ and a plan $\boldsymbol{\mu} \in \mathcal{P}\left(\mathbb{R}^{d} \times \mathbb{R}^{d}\right)$ with marginals $\mu_{0}, \mu_{1}$ such that

$$
\int_{\mathbb{R}^{d} \times \mathbb{R}^{d}} c_{\hat{\alpha}}\left(x_{0}, x_{1}\right) \mathrm{d} \boldsymbol{\mu}<\int_{\Gamma} \int_{0}^{1} L_{\hat{\alpha}}(t, \gamma(t), \dot{\gamma}(t)) \mathrm{d} t \mathrm{~d} \boldsymbol{\eta}(\gamma)-\delta .
$$

By using the measurable selection theorem stated in Lemma 7.6, we can find a $\boldsymbol{\mu}$-measurable map $\boldsymbol{\omega}: X_{\alpha} \rightarrow$ $\Gamma_{\alpha}$ such that for every $x_{0}, x_{1}$ the curve $\gamma=\boldsymbol{\omega}\left(x_{0}, x_{1}\right) \in \mathrm{AC}_{2}\left([0,1] ; \mathbb{R}^{d}\right)$ satisfies

$$
\gamma(i)=x_{i}, \int_{0}^{1} \hat{M} \alpha(t, \gamma(t)) \mathrm{d} t<\infty, \int_{0}^{1} L_{\hat{\alpha}}(t, \gamma(t), \dot{\gamma}(t)) \leq c_{\hat{\alpha}}\left(x_{0}, x_{1}\right)+\delta .
$$

It is clear that the plan $\boldsymbol{\eta}^{\prime}:=\boldsymbol{\omega}_{\sharp} \boldsymbol{\mu}$ contradicts (7.50). 
Claim 4: $(i v) \Rightarrow(i)$

Let $\left(m^{\prime}, \boldsymbol{v}^{\prime}\right)$ be in $\mathrm{CE}_{2, p}\left(Q ; \mu_{0}, \mu_{1}\right)$. By Theorem 7.1 we can find a plan $\boldsymbol{\eta}^{\prime} \in \mathcal{P}_{2, p}(\Gamma)$ tightened to $\left(m^{\prime}, \boldsymbol{v}^{\prime}\right)$ and thus satisfying $\left(\mathrm{e}_{i}\right)_{\sharp} \boldsymbol{\eta}^{\prime}=\mu_{i}, \hat{M} \alpha(\cdot, \gamma) \in L^{1}(0,1)$ for $\boldsymbol{\eta}^{\prime}$-a.e. $\gamma$. Setting $\boldsymbol{\mu}^{\prime}:=\left(\mathrm{e}_{0}, \mathrm{e}_{1}\right)_{\sharp} \boldsymbol{\eta}^{\prime}$ we have

$$
\begin{aligned}
\mathcal{L}_{\alpha}\left(m^{\prime}, \boldsymbol{v}^{\prime}\right) & =\int_{Q} L_{\alpha}\left(t, x, \boldsymbol{v}^{\prime}\right) m^{\prime} \mathrm{d} x \mathrm{~d} t=\int_{Q} L_{\hat{\alpha}}\left(t, x, \boldsymbol{v}^{\prime}\right) m^{\prime} \mathrm{d} x \mathrm{~d} t=\int_{\Gamma} \int_{0}^{1} L_{\hat{\alpha}}(t, \gamma(t), \dot{\gamma}(t)) \mathrm{d} t \mathrm{~d} \boldsymbol{\eta}^{\prime}(\gamma) \\
& \geq \int_{\mathbb{R}^{d} \times \mathbb{R}^{d}} c_{\hat{\alpha}}(\gamma(0), \gamma(1)) \mathrm{d} \boldsymbol{\eta}^{\prime}(\gamma)=\int_{\mathbb{R}^{d} \times \mathbb{R}^{d}} c_{\hat{\alpha}}\left(x_{0}, x_{1}\right) \mathrm{d} \boldsymbol{\mu}^{\prime}\left(x_{0}, x_{1}\right) \geq \int_{\mathbb{R}^{d} \times \mathbb{R}^{d}} c_{\hat{\alpha}}\left(x_{0}, x_{1}\right) \mathrm{d} \boldsymbol{\mu}\left(x_{0}, x_{1}\right) \\
& =\int_{\Gamma} \int_{0}^{1} L_{\hat{\alpha}}(t, \gamma(t), \dot{\gamma}(t)) \mathrm{d} t \mathrm{~d} \boldsymbol{\eta}(\gamma)=\int_{Q} L_{\hat{\alpha}}(t, x, \boldsymbol{v}) m \mathrm{~d} x \mathrm{~d} t=\mathcal{L}_{\alpha}(m, \boldsymbol{v})
\end{aligned}
$$

Thus $(m, \boldsymbol{v})$ minimizes the modified Lagrangian dynamic cost and we can apply Theorem 6.16.

\section{A Appendix}

\section{A.1 Minimax and duality}

Let $\mathbb{A}, \mathbb{B}$ be convex sets of some vector spaces and let us suppose that $\mathbb{B}$ is endowed with some Hausdorff topology. Let $\mathcal{L}: \mathbb{A} \times \mathbb{B} \rightarrow \mathbb{R}$ be a saddle function satisfying

$$
\begin{aligned}
a \mapsto \mathcal{L}(a, b) & \text { is concave in } \mathbb{A} \text { for every } b \in \mathbb{B}, \\
b \mapsto \mathcal{L}(a, b) & \text { is convex in } \mathbb{B} \text { for every } a \in \mathbb{A} .
\end{aligned}
$$

It is always true that

$$
\inf _{b \in \mathbb{B}} \sup _{a \in \mathbb{A}} \mathcal{L}(a, b) \geq \sup _{a \in \mathbb{A}} \inf _{b \in \mathbb{B}} \mathcal{L}(a, b) .
$$

The next result provides an important sufficient condition to guarantee the equality in (A.3): we use a formulation which is slightly more general than the statement of [30, Thm. 3.1], but it follows by the same argument. We reproduce here the main part of the proof for the easy of the reader.

Theorem A.1 (Von Neumann) Let us suppose that A.1 , A.2 hold and that there exists $a_{\star} \in \mathbb{A}$ and $C_{\star}>\sup _{a \in \mathbb{A}} \mathcal{L} \mathcal{L}(a, b)$ such that

$$
\begin{aligned}
\mathbb{B}_{\star}: & =\left\{b \in \mathbb{B}: \mathcal{L}\left(a_{\star}, b\right) \leq C_{\star}\right\} \quad \text { is not empty and compact in } \mathbb{B}, \\
b & \mapsto \mathcal{L}(a, b) \text { is lower semicontinuous in } \mathbb{B}_{\star} \text { for every } a \in \mathbb{A} .
\end{aligned}
$$

Then

$$
\min _{b \in \mathbb{B}} \sup _{a \in \mathbb{A}} \mathcal{L}(a, b)=\sup _{a \in \mathbb{A}} \inf _{b \in \mathbb{B}} \mathcal{L}(a, b) .
$$

Proof. Let $s:=\operatorname{supinf}_{a \in \mathbb{A}} \mathcal{L}(a, b)$ and let $\mathbb{B}_{a}:=\{b \in \mathbb{B}: \mathcal{L}(a, b) \leq s\}, \mathbb{B}_{a \star}:=\{b \in \mathbb{B}: \mathcal{L}(a, b) \leq s\}$. If $A \subset \mathbb{A}$ is a collection containing $a_{\star}$ then

$$
\mathbb{B}_{A}=\bigcap_{a \in A} \mathbb{B}_{a}=\bigcap_{a \in A} \mathbb{B}_{a \star}
$$

so that $\mathbb{B}_{A}$ is a (possibly empty) compact set. The thesis follows if we check that $\mathbb{B}_{\mathbb{A}}$ contains a point $\bar{b}$, since in that case $\min _{b \in \mathbb{B}} \sup _{a \in \mathbb{A}} \mathcal{L}(a, b) \leq \sup _{a \in \mathbb{A}} \mathcal{L}(a, \bar{b}) \leq s$ by construction.

Since $\mathbb{B}_{A}$ are compact whenever $a_{\star} \in A$, it is sufficient to prove that for every finite collection $A=$ $\left\{a_{1}, \cdots, a_{n}\right\}$ containing $a_{\star}$ the intersection $B_{A}$ is not empty. To this aim, since $b \mapsto \mathcal{L}\left(a_{k}, b\right)$ are convex functions, [30, Lemma 2.1] yields

$$
\min _{b \in \mathbb{B}_{\star}} \sup _{1 \leq k \leq n} \mathcal{L}\left(a_{k}, b\right)=\min _{b \in \mathbb{B}_{\star}} \sum_{k=1}^{N} \chi_{k} \mathcal{L}\left(a_{k}, b\right)
$$

for a suitable choice of nonnegative coefficients $\chi_{k} \in[0,1]$ with $\sum_{k=1}^{n} \chi_{k}=1$. We thus get by concavity

$$
\min _{b \in \mathbb{B}_{\star}} \sum_{k=1}^{n} \mathcal{L}\left(a_{k}, b\right) \leq \min _{b \in \mathbb{B}_{\star}} \mathcal{L}\left(\sum_{k=1}^{N} \chi_{k} a_{k}, b\right) \leq s .
$$




\section{A.2 Convergence in measure}

Lemma A.2 Let $u_{n}, u \in L^{0}(\Omega, \mathfrak{m}), n \in \mathbb{N}$. The following properties are equivalent:

(a) $\lim _{n \uparrow+\infty} d\left(u_{n}, u\right)=0$;

(b) $u_{n}$ converges to $u$ in $\varrho$-measure, i.e.

$$
\text { for every } \varepsilon>0: \quad \lim _{n \uparrow+\infty} \varrho\left(\left\{x \in \Omega:\left|u_{n}(x)-u(x)\right| \geq \varepsilon\right\}\right)=0
$$

(c) $u_{n}$ converges to $u$ in measure, according to (4.2).

Moreover, if $\zeta: \mathbb{R} \rightarrow \mathbb{R}$ is any continuous and strictly increasing map, $u_{n}$ converges to $u$ in measure if and only if $\zeta \circ u_{n}$ converges in measure to $\zeta \circ u$.

Proof. The equivalence between (a) and (b) is well known (see e.g. [7, 4.7.60]).

(b) $\Rightarrow$ (c): since $\rho>0 \mathfrak{m}$-a.e., $\mathfrak{m}$ is absolutely continuous w.r.t. $\varrho$ with density $\rho^{-1}$, so that the finite measure $\mathfrak{m}_{\mid}$satisfies [7, Theorem 2.5.7]

$$
\forall \eta>0 \quad \exists \delta>0: \quad \mathfrak{m}(A)<\eta \quad \text { if } A \subset F, \varrho(A)<\delta .
$$

(A.7) then yields (4.2).

(c) $\Rightarrow$ (b): we consider a measurable partition $\left(F_{k}\right)_{k \in \mathbb{N}}$ of $\Omega$ with $\mathfrak{m}\left(F_{k}\right)<\infty$ for every $k \in \mathbb{N}$. If (4.2) holds, setting $A_{n}:=\left\{x \in \Omega:\left|u_{n}(x)-u(x)\right|>\varepsilon\right\}$ we get for every $k \in \mathbb{N}$

$$
\lim _{n \rightarrow \infty} \varrho\left(A_{n} \cap F_{k}\right)=0
$$

since $\varrho \leq \mathfrak{m}$, so that

$$
\lim _{n \rightarrow \infty} \varrho\left(A_{n}\right)=\lim _{n \rightarrow \infty} \sum_{k \in \mathbb{N}} \varrho\left(A_{n} \cap F_{k}\right)=\sum_{k \in \mathbb{N}} \lim _{n \rightarrow \infty} \varrho\left(A_{n} \cap F_{k}\right)=0
$$

where the interchange between the series and the integral is justified by the uniform domination

$$
\varrho\left(A_{n} \cap F_{k}\right) \leq \varrho\left(F_{k}\right), \quad \sum_{k \in \mathbb{N}} \varrho\left(F_{k}\right)=1 .
$$

The last statement of the Lemma concerning the composition map $f \mapsto \zeta \circ f$ is well known (see e.g. 7, Cor. 2.2.6]).

\section{A.3 Positive bilinear functionals}

Theorem A.3 Let $T: C_{c}(\mathbb{R}) \times C_{c}\left(\mathbb{R}^{d}\right) \rightarrow \mathbb{R}$ be a bilinear map which is positive on pairs of positive functions. Then there exists a positive Radon measure $\vartheta$ on $\mathbb{R} \times \mathbb{R}^{d}$ such that $T(\phi, \psi)=\int_{\mathbb{R}^{\prime} \mathbb{R}^{d}} \varphi(t) \psi(x) \mathrm{d} \vartheta(t, x)$ for every $\varphi \in C_{c}^{0}(\mathbb{R}), \psi \in C_{c}^{0}\left(\mathbb{R}^{d}\right)$.

Proof. Let us briefly sketch the proof. To this aim, we denote by $y=(t, x)$ the element in $\mathbb{R}^{d+1}$ and we first define a linear functional $T$ on functions $\psi \in C_{c}^{0}\left(\mathbb{R} \times \mathbb{R}^{d+1}\right)$ admitting the representation

$$
\psi(u, y)=\sum_{i=1}^{I} \zeta_{i}(u) \varphi_{i}(y) \quad \zeta_{i} \in C_{c}^{0}(\mathbb{R}), \varphi_{i} \in C_{c}^{0}\left(\mathbb{R}^{d+1}\right) .
$$

The natural definition would be

$$
T(\psi):=\sum_{i=1}^{I} T_{\zeta_{i}}\left(\varphi_{i}\right)
$$

Let us check that if $\psi \geq 0$ then the above expression is positive: this would imply that (A.9) is independent of the representation of $\psi$ given by (A.8).

In order to prove this property, let us consider a nonnegative symmetric regularization kernel $h \in C_{c}^{\infty}(\mathbb{R})$ with $\operatorname{supp}(h) \subset(-1 / 2,1 / 2), \int_{\mathbb{R}} h(t) \mathrm{d} t=1, h_{\tau}(t):=\tau^{-1} h(t / \tau)$. We select an integer $K>0$ sufficiently big so that $\operatorname{supp}\left(\zeta_{i}\right) \subset(-K+1, K-1)$ for every $i=1,2, \ldots, I$ and we fix a nonnegative function $\zeta_{K} \in C_{c}^{0}(\mathbb{R})$ such that $\zeta_{K}(t) \equiv 1$ on $K$. 
For every $\tau \in(0,1)$ we set

$$
\zeta_{i, \tau}(t):=\frac{K}{N} \sum_{k=-N K}^{N K} \zeta_{i}(k / N) h_{\tau}(t-k / N)
$$

Notice that $\zeta_{i, \tau}$ is supported in $(-K+1 / 2, K-1 / 2)$; moreover, since $\lim _{\tau \downarrow 0} \zeta_{i} * h_{\tau}=\zeta_{i}$ uniformly, $\zeta_{i}$ are uniformly continuous and $\operatorname{supp}\left(\zeta_{i} * h_{\tau}\right) \subset(-K+1 / 2, K-1 / 2)$ for $\tau<1$, for every $\varepsilon>0$ we can find $N$ sufficiently big so that

$$
\sup _{t \in \mathbb{R}}\left|\zeta_{i, \tau}(t)-\zeta_{i}(t)\right| \leq \varepsilon, \quad \operatorname{supp}\left(\zeta_{i, \tau}-\zeta_{i}\right) \subset(-K+1 / 2, K-1 / 2), \quad-\varepsilon \zeta_{K} \leq\left|\zeta_{i, \tau}-\zeta_{i}\right| \leq \varepsilon \zeta_{K} .
$$

It follows by the positivity of $T$. that for every nonnegative $\varphi \in C_{c}^{0}\left(\mathbb{R}^{d+1}\right)$

$$
-\varepsilon T_{\zeta_{K}}(\varphi) \leq\left|T_{\zeta_{i, \tau}}(\varphi)-T_{\zeta_{i}}(\varphi)\right| \leq \varepsilon T_{\zeta_{K}}(\varphi)
$$

On the other hand

$$
\begin{aligned}
\sum_{i=1}^{I} T_{\zeta_{i, \tau}}\left(\varphi_{i}\right) & =\sum_{i=1}^{I} \sum_{k=-K N}^{K N} \zeta_{i}(k / N) T_{h_{\tau}(\cdot-k / N)}\left(\varphi_{i}\right)=\sum_{k=-K N}^{K N} \sum_{i=1}^{I} \zeta_{i}(k / N) T_{h_{\tau}(\cdot-k / N)}\left(\varphi_{i}\right) \\
& =\sum_{k=-K N}^{K N} T_{h_{\tau}(\cdot-k / N)}\left(\sum_{i=1}^{N}\left(\zeta_{i}(k / N) \varphi_{i}\right)\right)=\sum_{k=-K N}^{K N} T_{h_{\tau}(\cdot-k / N)}(\psi(k / N, \cdot)) \geq 0
\end{aligned}
$$

where in the last inequality we used the fact that $h$ is nonnegative and $y \mapsto \psi(k / N, y) \geq 0$ by assumption. Combining the last inequality with (A.12) we conclude that (A.9) defines a positive linear functionals on the algebra of functions admitting the decomposition (A.8). Since this algebra is uniformly dense in $C_{c}^{0}(\mathbb{R})$ we deduce that there exists a unique Radon measure $\vartheta$ on $\mathbb{R} \times \mathbb{R}^{d+1}$ representing $T$ as in (5.55).

\section{References}

[1] Yves Achdou, Fabio Camilli, and Italo Capuzzo-Dolcetta. Mean field games: numerical methods for the planning problem. SIAM J. Control Optim., 50(1):77-109, 2012.

[2] Luigi Ambrosio and Alessio Figalli. Geodesics in the space of measure-preserving maps and plans. Arch. Ration. Mech. Anal., 194(2):421-462, 2009.

[3] Luigi Ambrosio, Nicola Gigli, and Giuseppe Savaré. Gradient flows in metric spaces and in the space of probability measures. Lectures in Mathematics ETH Zürich. Birkhäuser Verlag, Basel, second edition, 2008.

[4] Jean-David Benamou and Yann Brenier. A computational fluid mechanics solution to the MongeKantorovich mass transfer problem. Numer. Math., 84(3):375-393, 2000.

[5] Jöran Bergh and Jörgen Löfström. Interpolation spaces. An introduction. Springer-Verlag, Berlin-New York, 1976. Grundlehren der Mathematischen Wissenschaften, No. 223.

[6] Patrick Bernard and Boris Buffoni. Optimal mass transportation and Mather theory. J. Eur. Math. Soc. (JEMS), 9(1):85-121, 2007.

[7] V. I. Bogachev. Measure theory. Vol. I. Springer-Verlag, Berlin, 2007.

[8] V. I. Bogachev. Measure theory. Vol. II. Springer-Verlag, Berlin, 2007.

[9] Pierre Cardaliaguet. Weak solutions for first order mean field games with local coupling. In Analysis and geometry in control theory and its applications, volume 11 of Springer INdAM Ser., pages 111-158. Springer, Cham, 2015.

[10] Pierre Cardaliaguet, Guillaume Carlier, and Bruno Nazaret. Geodesics for a class of distances in the space of probability measures. Calculus of Variations and Partial Differential Equations, 48(2,3):395420, 2013.

[11] Pierre Cardaliaguet and P. Jameson Graber. Mean field games systems of first order. ESAIM Control Optim. Calc. Var., 21(3):690-722, 2015. 
[12] Pierre Cardaliaguet, P. Jameson Graber, Alessio Porretta, and Daniela Tonon. Second order mean field games with degenerate diffusion and local coupling. NoDEA Nonlinear Differential Equations Appl., $22(5): 1287-1317,2015$.

[13] Pierre Cardaliaguet, Alpár R. Mészáros, and Filippo Santambrogio. First order mean field games with density constraints: pressure equals price. SIAM J. Control Optim., 54(5):2672-2709, 2016.

[14] C. Castaing and M. Valadier. Convex analysis and measurable multifunctions. Springer-Verlag, Berlin, 1977. Lecture Notes in Mathematics, Vol. 580.

[15] Lénaï c Chizat, Gabriel Peyré, Bernhard Schmitzer, and François-Xavier Vialard. Unbalanced optimal transport: Dynamic and Kantorovich formulations. J. Funct. Anal., 274(11):3090-3123, 2018.

[16] Ivar Ekeland and Roger Témam. Convex analysis and variational problems, volume 28 of Classics in Applied Mathematics. Society for Industrial and Applied Mathematics (SIAM), Philadelphia, PA, english edition, 1999. Translated from the French.

[17] Irene Fonseca and Giovanni Leoni. Modern methods in the calculus of variations: $L^{p}$ spaces. Springer Monographs in Mathematics. Springer, New York, 2007.

[18] Jean-Michel Lasry and Pierre-Louis Lions. Jeux à champ moyen. I. Le cas stationnaire. C. R. Math. Acad. Sci. Paris, 343(9):619-625, 2006.

[19] Jean-Michel Lasry and Pierre-Louis Lions. Jeux à champ moyen. II. Horizon fini et contrôle optimal. C. R. Math. Acad. Sci. Paris, 343(10):679-684, 2006.

[20] Jean-Michel Lasry and Pierre-Louis Lions. Mean field games. Jpn. J. Math., 2(1):229-260, 2007.

[21] Christian Léonard. From the Schrödinger problem to the Monge-Kantorovich problem. J. Funct. Anal., 262(4):1879-1920, 2012.

[22] Matthias Liero, Alexander Mielke, and Giuseppe Savaré. Optimal entropy-transport problems and a new Hellinger-Kantorovich distance between positive measures. Invent. Math., 211(3):969-1117, 2018.

[23] P.-L. Lions. Cours au collège de france. année 2009-2010. Technical report, www.college-de-france.fr.

[24] Robert J. McCann. A convexity principle for interacting gases. Adv. Math., 128(1):153-179, 1997.

[25] F. Otto and C. Villani. Generalization of an inequality by Talagrand and links with the logarithmic Sobolev inequality. J. Funct. Anal., 173(2):361-400, 2000.

[26] Felix Otto. The geometry of dissipative evolution equations: the porous medium equation. Comm. Partial Differential Equations, 26(1-2):101-174, 2001.

[27] Alessio Porretta. On the planning problem for a class of mean field games. C. R. Math. Acad. Sci. Paris, 351(11-12):457-462, 2013.

[28] Alessio Porretta. On the planning problem for the mean field games system. Dyn. Games Appl., $4(2): 231-256,2014$.

[29] R. Rossi, G. Savaré, A. Segatti, and U. Stefanelli. Weighted Energy-Dissipation principle for gradient flows in metric spaces. ArXiv e-prints, 2018.

[30] Stephen Simons. Minimax and monotonicity, volume 1693 of Lecture Notes in Mathematics. SpringerVerlag, Berlin, 1998.

[31] Cédric Villani. Optimal transport, volume 338 of Grundlehren der Mathematischen Wissenschaften [Fundamental Principles of Mathematical Sciences]. Springer-Verlag, Berlin, 2009. Old and new. 